\title{
NUMERICAL NAVIER-STOKES SOLUTIONS OF SUPERSONIC SLOT INJECTION PROBLEMS \\ by
}

\author{
Sung Joon Yoon
}

\begin{abstract}
Dissertation submitted to the Faculty of the
Virginia Polytechnic Institute and State University

in partial fulfillment of the requirements for the degree of

Doctor of Philosophy

in

Aerospace Engineering
\end{abstract}

APPROVED:

Dr. Joseph A. Schetz, Chairman

Dr. Bernard Grossman

Dr. Koger L. Jónpson

Dr. Antoni K. Jakub6wski

Dr. Robert W. Walters

April 1988

Blacksburg, Virginia 


\title{
NUMERICAL NAVIER-STOKES SOLUTIONS OF SUPERSONIC SLOT INJECTION PROBLEMS
}

by

\author{
Sung Joon Yoon \\ Dr. Joseph A. Schetz, Chairman \\ Aerospace Engineering
}

(ABSTRACT)

Supersonic slot injection problems were studied by a finite volume method. The numerical technique used is the upwind method of Roe's flux difference splitting (FDS) with vertical line Gauss-Seidel relaxation applied to the thin layer Navier-Stokes equations. To test the accuracy of the numerical methods without the complications and uncertainties of turbulence modelling, two sample cases were chosen with laminar flows. The sample problems were the compressible laminar boundary layer flow over a flat plate and the laminar boundary layer shock interaction problem. For these problems, both the results from Roe's FDS and van Leer's flux vector splitting (FVS) are compared with exact solutions and experimental data. For the sample problems, comparisons showed that Roe's FDS method is more accurate than van Leer's FVS method. Because of the very complicated wave patterns and strong viscous-inviscid interaction produced by supersonic slot injection, an adaptive grid based on the equidistribution law was combined with the solution algorithm. The results from Roe's FDS method with the adaptive grid showed good results for the supersonic slot injection over a flat 
plate. For the slot injection over a 10 degree wedge surface case, there is a significant difference between the numerical and experimental wall pressure distribution. Some potential reasons for the discrepancy including 3D effects and / or transition in the reattachment region in the experiments and possibly a need for a much finer grid in the calculations are discussed. 


\section{Acknowledgements}

The author wishes to express his sincere appreciation to his advisor, Dr. J. A. Schetz who gave guidance, advice and encouragement to this study. Without his energetic support, it would have been difficult to finish this work. The author wishes to thank Dr. B. Grossman, Dr. R. L. Simpson, Dr. A. K. Jakubowski and Dr. R. W. Walters for serving on the Advisory Committee and their comments and suggestions on this work.

He also wishes to thank Dr. H. E. Gilreath of APL for supplying the experimental conditions of his slot injection experiments.

Very Special thanks are given to his wife for her endurance and encouragement throughout this study. 


\section{Table of Contents}

1.0 Introduction $\ldots \ldots \ldots \ldots \ldots \ldots \ldots \ldots \ldots \ldots \ldots \ldots \ldots \ldots \ldots \ldots \ldots$

2.0 Formulation $\ldots \ldots \ldots \ldots \ldots \ldots \ldots \ldots \ldots \ldots \ldots \ldots \ldots \ldots \ldots \ldots$

2.1 Governing Equations $\ldots \ldots \ldots \ldots \ldots \ldots \ldots \ldots \ldots \ldots \ldots \ldots \ldots$

2.2 Adaptive Grid $\ldots \ldots \ldots \ldots \ldots \ldots \ldots \ldots \ldots \ldots \ldots \ldots \ldots \ldots \ldots \ldots \ldots$

3.0 Space Discretization $\ldots \ldots \ldots \ldots \ldots \ldots \ldots \ldots \ldots \ldots \ldots \ldots \ldots \ldots \ldots \ldots \ldots$

3.1 Finite Volume Formulation $\ldots \ldots \ldots \ldots \ldots \ldots \ldots \ldots \ldots \ldots \ldots \ldots \ldots \ldots \ldots \ldots \ldots$

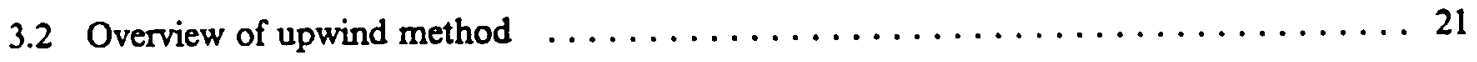

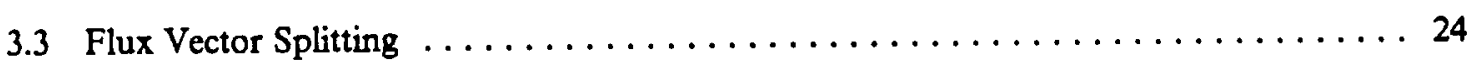

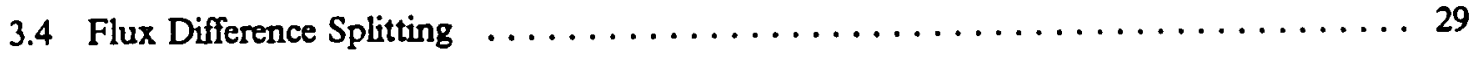

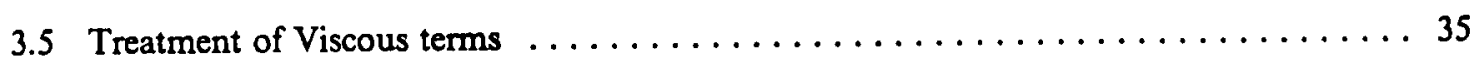

4.0 Time Integration $\ldots \ldots \ldots \ldots \ldots \ldots \ldots \ldots \ldots \ldots \ldots \ldots \ldots \ldots \ldots \ldots \ldots \ldots$

4.1 Backward Euler Time Integration $\ldots \ldots \ldots \ldots \ldots \ldots \ldots \ldots \ldots \ldots \ldots \ldots \ldots \ldots$

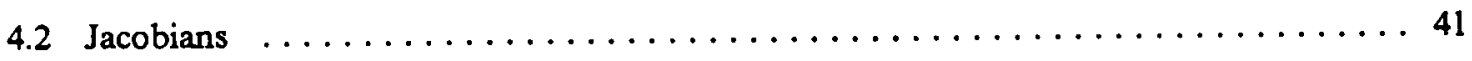

4.2 .1 Jacobians of Inviscid Terms $\ldots \ldots \ldots \ldots \ldots \ldots \ldots \ldots \ldots \ldots \ldots \ldots \ldots \ldots$ 


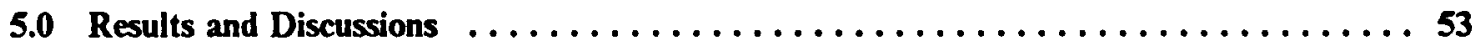

5.1 Compressible Laminar Boundary Layer over a Flat Plate $\ldots \ldots \ldots \ldots \ldots \ldots \ldots$

5.2 Laminar Boundary Layer - Shock Interaction $\ldots \ldots \ldots \ldots \ldots \ldots \ldots \ldots$

5.3 Supersonic Slot Injection Problem $\ldots \ldots \ldots \ldots \ldots \ldots \ldots \ldots \ldots \ldots \ldots \ldots \ldots \ldots \ldots \ldots \ldots$

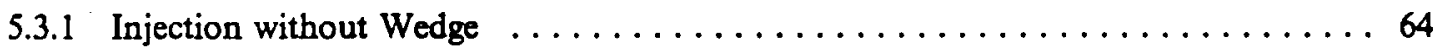

5.3.2 Injection over a 10 -Degree Wedge $\ldots \ldots \ldots \ldots \ldots \ldots \ldots \ldots \ldots \ldots \ldots$

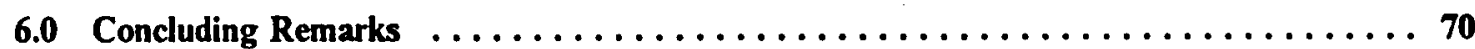

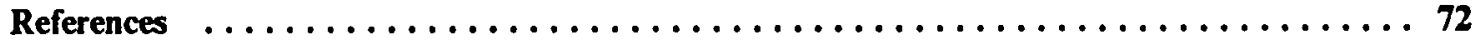

Figures $\ldots \ldots \ldots \ldots \ldots \ldots \ldots \ldots \ldots \ldots \ldots \ldots \ldots \ldots \ldots \ldots \ldots \ldots \ldots \ldots \ldots$

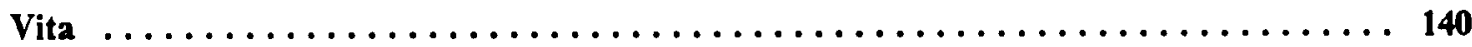




\section{List of Illustrations}

Figure 1. Schematic representation of supersonic slot injection problems. . 82

Figure 2. A finite volume element. $\ldots \ldots \ldots \ldots \ldots \ldots \ldots \ldots \ldots$

Figure 3. Schematic of compressible laminar boundary layer problem. . . 84

Figure 4. $41 \times 41$ grid for compressible laminar boundary layer problem. . 85

Figure 5. Velocity profiles in the boundary layer, $41 \times 41$ grid (FDS-Roe). 86

Figure 6. Temperature profiles in the boundary layer, $41 \times 41$ grid (FDS-Roe). ....................... 87

Figure 7. The effect of grid refinement, $\mathrm{X} / \mathrm{L}=.5$ - velocity (FDS-Roe). $\quad .88$

Figure 8. The effect of grid refinement, $\mathrm{X} / \mathrm{L}=.5$ - temperature (FDS-Roe).

Figure 9. Convergence history for the compressible laminar boundary layer,

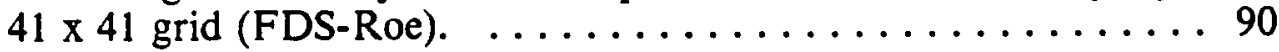

Figure 10. Velocity profiles in the boundary layer, $41 \times 41$ grid (FVS-van Leer).

Figure 11. Temperature profiles in the boundary layer, $41 \times 41$ grid (FVS-van Leer)

Figure 12. The effect of grid refinement, $\mathrm{X} / \mathrm{L}=.5$ - velocity (FVS-van Leer). 93

Figure 13. The effect of grid refinement, $\mathrm{X} / \mathrm{L}=.5-$ temperature (FVS-van Leer). ....................... 94

Figure 14. A sketch of laminar boundary layer-shock interaction problem( from MacCormack and Baldwin). ............. 95 
Figure $15.32 \times 57$ grid for laminar boundary layer-shock interaction problem. ........................ 96

Figure 16. Wall pressure distribution for the laminar boundary layer - shock interaction problem (FDS-Roe). $\ldots \ldots \ldots \ldots \ldots \ldots \ldots \ldots$

Figure 17. Wall skin friction coefficient distribution for the laminar boundary layer - shock interaction problem (FDS-Roe). . . . . . . 98

Figure 18. Wall pressure distribution for the laminar boundary layer - shock interaction problem (FVS-van Leer). . . . . . . . . . . 99

Figure 19. Wall skin friction coefficient distribution for the laminar boundary layer - shock interaction problem (FVS-van Leer). . . 100

Figure 20. Grid refinement for the laminar boundary layer - shock

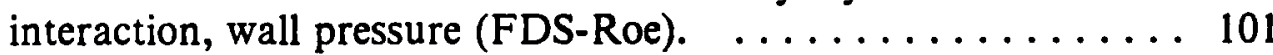

Figure 21. Grid refinement for the laminar boundary layer - shock

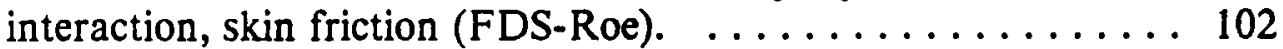

Figure 22. Pressure contour for the laminar boundary layer - shock interaction, $63 \times 57$ grid (FDS-Roe).

Figure 23. Comparison of computations with experiment for the laminar boundary layer - shock interaction problem. . . . . . . . . 104

Figure 24. Pressure contour for the laminar boundary layer - shock interaction, $63 \times 57$ grid (FVS-van Leer).

Figure 25. Grid refinement for the laminar boundary layer - shock interaction, wall pressure (FVS-van Leer).

Figure 26. Grid refinement for the laminar boundary layer - shock interaction, skin friction (FVS-van Leer).

Figure 27. $51 \times 70$ grid for supersonic slot injection problem (without wedge).

Figure 28. Wall pressure distribution for supersonic slot injection problem (without wedge).

Figure 29. Skin friction distribution for supersonic slot injection problem (without wedge). .................... 110 
Figure 30. Pressure contour for supersonic slot injection problem (without wedge) $-51 \times 70$ grid.

Figure 31. Density contour for supersonic slot injection problem (without wedge) $-51 \times 70$ grid.

Figure 32. Wall pressure distribution for supersonic slot injection problem ( $51 \times 70$ grid ) - double precision vs. single precision.

Figure 33. Skin friction distribution for supersonic slot injection problem ( $51 \times 70$ grid ) - double precision vs. single precision.

Figure 34. Convergence history for supersonic slot injection problem ( $51 \mathrm{x}$ 70 grid ) - double precision vs. single precision.

Figure 35. $81 \times 90$ grid for supersonic slot injection problem (without wedge).

Figure 36. Grid refinement for supersonic slot injection problem (without wedge) - wall pressure.

Figure 37. Grid refinement for supersonic slot injection problem (without wedge) - skin friction.

Figure 38. Pressure contour for supersonic slot injection problem (without wedge) $-81 \times 90$ grid.

Figure 39. Density contour for supersonic slot injection problem (without wedge) $-81 \times 90$ grid.

Figure 40. Wall pressure distribution for supersonic slot injection problem (without wedge), inviscid - $81 \times 90$ grid.

Figure 41. Pressure contour for supersonic slot injection problem (without wedge), inviscid - $81 \times 90$ grid.

Figure 42. Density contour for supersonic slot injection problem (without wedge), inviscid - $81 \times 90$ grid.

Figure 43. Convergence history for supersonic slot injection problem (without wedge), inviscid - $81 \times 90$ grid.

Figure 44. $81 \times 90$ adaptive grid for supersonic slot injection problem (without wedge). 
Figure 45. Pressure contour for supersonic slot injection problem (without wedge) - $81 \times 90$ adaptive grid.

Figure 46. Density contour for supersonic slot injection problem (without wedge) $-81 \times 90$ adaptive grid.

Figure 47. Wall pressure distribution for supersonic slot injection problem (without wedge) $-81 \times 90$ adaptive grid. $\ldots \ldots \ldots \ldots \ldots 128$

Figure 48. Wall skin friction distribution for supersonic slot injection problem (without wedge) $-81 \times 90$ adaptive grid.

Figure 49. Comparison of experimental and numerical flowfield visualization (without wedge) - interferograms.

Figure 50. Comparison of experimental and numerical flowfield visualization (without wedge) - Schlieren. . . . . . . . . .

Figure $51.65 \times 70$ grid for supersonic slot injection over a 10 degree wedge surface.

Figure 52. Pressure contour for supersonic slot injection over a 10 degree wedge surface.

Figure 53. Density contour for supersonic slot injection over a 10 degree wedge surface.

Figure 54. Comparison of wall pressure distribution between numerical and experimental data (with 10 degree wedge).

Figure 55. Skin friction distribution (with 10 degree wedge) $-65 \times 70$ grid. 136

Figure 56. The effect of the location of outflow boundary - skin friction. . 137

Figure 57. The effect of the location of outflow boundary - pressure. . . 138

Figure 58. Comparison of experimental and numerical flowfield visualization (with 10 degree wedge) - Schlieren. 


\title{
List of Symbols
}

\author{
$\hat{A}, \hat{B} \ldots \ldots . . . . . .$. inviscid Jacobian matrix \\ c....................speed of sound \\ C ..................constant in Sutherland's formula \\ $c_{f} \ldots \ldots . . . . . . . . . . . . . . . s k i n$ friction coefficient \\ e....................total energy
}

$\bar{f}$....................normalized weight function

$F, G$.............inviscid flux vectors in the $\mathrm{x}$ - and $\mathrm{y}$ - direction

$F_{v}, G_{v} \ldots \ldots . . . . . v i s c o u s$ flux vectors in the $\mathrm{x}$ - and $\mathrm{y}$ - direction

H ..................stagnation enthalpy

I....................identity matrix

$\hat{H}$...................viscous Jacobian matrix

J..................Jacobian associated with coordinate transformation

L...................reference length

$\bar{L}, \bar{R}$...........matrices whose columns are left and right eigenvectotrs 
M ..................Mach number

$\tilde{M}$..................Jacobian matrix based on Roe averaging

N.................total number of grid points

$n_{x}, n_{y} \ldots \ldots . . . . . \mathrm{x}-$ and $\mathrm{y}$ - components of unit vector normal to $\xi=$ const. lines

p ...................static pressure

Pr ................Prandtl number

q ….............. $\left(=\sqrt{u^{2}+v^{2}}\right)$, speed

$\mathrm{Q}$...................conservative variable

R ..................steady state residual

Re................ Reynolds number

s.....................arc length

$\Delta \mathrm{S}$...............length of cell interface length

t....................time

T...................static temperature or transformation matrix

$\Delta t$..................time step

$t_{x}, t_{y} \ldots \ldots . . . . . \mathrm{x}-$ and $\mathrm{y}$ - components of unit vector normal to $\eta=$ const. lines

$\mathrm{u}, \mathrm{v}$..............velocity component in the $\mathrm{x}$ - and $\mathrm{y}$ - direction

W .................similarity variable

w ..................weighting function

$\mathrm{x}, \mathrm{y}$.................Cartesian coordinate system

Greek symbols 
$\gamma$....................ratio of specific heats

$\Delta$.................. difference of right and left cell interface

$\xi, \eta \ldots . . . . . . . . . . . . b o d y$ fitted coordinate system

$\lambda$...................second viscous coefficient

$\Lambda$..................eigenvalues of Jacobian matrix

$\mu \ldots . . . . . . . . . . . . . . . v v i s c o u s$ coefficient

$\rho$.....................density

$\tau$................ viscous shear stress

Subscripts

$\mathrm{j}, \mathrm{k}$..................indices of body fitted coordinate system

$\infty$..................free stream condition

o .....................stagnation condition

Superscripts

$\mathrm{n}$...................index of time level

....................Roe averaged value

-..................normal to the cell interface 


\subsection{Introduction}

Injection of a gas through a wall slot parallel to a supersonic airstream is of interest for various applications, including thermal protection, fuel injection in ramjet engines, energizing of the inner portion of boundary layers in adverse pressure gradients and skin friction reduction on high speed aircraft. Currently, there has been a renewed interest in the slot injection problem due to recent research and development in the field of hypersonic cruise aircraft.

Although there have been a large number of experimental research works on the supersonic slot injection problem (Refs. $1-5$ ), existing analytical solutions of the slot injection problem suffered from the unrealistic simplifications such as a boundary layer approximation (Refs. 6 - 7). For these problems, strong transverse pressure gradients invalidate the boundary layer assumption. The flow field associated with supersonic slot injection usually has a complicated wave structure and shows the strong interaction of oblique shock waves and shear layers (Refs. 1 - 2) as illustrated in Fig. 1. Within the knowledge of the author, 
numerical Navier - Stokes prior solutions of supersonic slot injection problem do not exist. To understand the physical phenomena of the slot injection problem, numerical approaches without any unrealistic approximations must be pursued beside the experimental works.

In past two decades, there have been remarkable advances in the field of computational fluid dynamics. Both the improvement of computer power and numerical algorithms contribute to the progess of computational fluid dynamics as described in Ref. 8. Concerning the advancements of computer hardware, recently developed supercomputers which have capability of vector computation have influenced greatly the programming structure (Ref. 9) and if properly vectorized, a speedup ratio of order of ten is not unusual (Ref. 10).

Almost all of the numerical methods for the Navier-Stokes equations to date are of the unsteady formulation (Ref. 99). Starting from the initial conditions, the unsteady equations are integrated in time until a steady state is eventually reached. One advantage of the unsteady formulation is that both steady and unsteady flow problems can be solved with this formulation.

The time integration may be performed explicitly or implicitly. The explicit method has advantages such as simple logic, smaller operation counts than implicit methods and easy and complete vectorization compared to implicit methods (Refs. 11 - 13). But, it has a definite disadvantage. Due to the stability condition, there is a severe time step limit in the explicit method. For viscous flow 
computations, which usually involve a very thin viscous layer which must be resolved, such a limitation is a serious problem for any practical engineering calculations. For example, Drummond (Ref. 14) calculated the reacting turbulent flowfield in an axisymmetric ramjet dump combustor by an explicit method, and a hundred thousand time steps were required for convergence. To alleviate the severe time step limit of the explicit scheme, Jameson and his colleagues (Ref. 15) proposed a Runge-Kutta time integration scheme which has a bigger allowable time step than classical explicit methods. On the other hand, an implicit method such as Beam-Warming's AF method has a less severe time step limitation than explicit method (Refs. 16 - 17). But, factorizarion -error destroyed the good convergence characteristics at large time steps (Ref. 18), and the optimum CFL number (dimensionless time step) is not too much greater than the explicit method (Ref. 19).

The methods mentioned above use a central differencing for the space discretization. This kind of central differencing needs extra numerical dissipation when a discontinuity exists in the flow field. This extra numerical dissipation, called artificial viscosity, is necessary for the central differencing to eliminate the odd-even decoupling and to stabilize the scheme to capture the discontinuity. To reduce the false dissipation introduced by the artificial viscosity, it is often necessary to adjust the artificial viscosity for every flow condition. This makes central differencing schemes less robust. 
To eliminate the problem of the artificial viscosity and to better model the physical wave propagation problems numerically, there was a great interest in the construction of upwind schemes due to its inherent capability to simulate the physical wave propagation mechanisms (Refs. $19-28$ ). The basic idea of the upwind method stems from the fact that for a hyperbolic equation such as Euler equations, information propagates along a preferential trajectory in time and space. The upwind methods utilize this fact by using non-centered discretization for this directional dependence. In this aspect, the upwind method is more mathematically sound and robust than the central differencing scheme. When approximating a hyperbolic system of conservation laws with upwind methods, there exist various different models. One is Flux Difference Splitting (FDS) which simulates the wave propagation by exact or approximate solutions of Riemann problem. The other is Flux Vector Splitting (FVS), in which the interaction of neighboring cells is accomplished by splitting the fluxes based on the local velocity distribution. Both of the methods show good shock resolution capability when applied to Euler equations (Refs. 29 - 32) and computation of real gas cases (Ref. 33). Recently proposed higher order upwind methods with a smaller truncation error have been applied to viscous flow computations such as Navier-Stokes Equations (Refs. 34 - 36) and Parabolized Navier-Stokes Equations (Refs. 37 - 39). The promising results showed accurate shock capturing and shear layer resolution. Recently, van Leer et al (Ref. 45) compared various Flux Vector Splitting (FVS) and Flux Differencing Splitting (FDS) 
schemes for viscous conical flow problems. Following their study, Roe's FDS has a smaller numerical dissipation than van Leer's FVS for that case.

In addition to improvements in the solution algorithms, progress in grid generation has also been rapid (Refs. 40 - 41). Among the various grid generation schemes, grid adaptation is the one of the most important concepts (Ref. 42). In contrast to the Navier-Stokes equations, which are a mathematical statement of physical laws which govern the fluid motion, there is no such physical law which can be applied to grid generation. In this regard, the work of Brackbill and Saltzman (Ref. 51) is noteworthy. They define a function which contains three measures of computational grid - smoothness, orthogonality and cell volume variation. A grid is generated by minimizing the resulting function with a variational formulation. Many of the proposed classical and adaptive grid generation schemes are related to the variational formulation. Anderson (Refs. 43 - 44) showed that elliptic grid generation schemes can be interpreted as an adaptive grid method with proper formulation of the weighting function, and he obtained good results for simple problems. If the grid smoothness and orthogonality are not included in the variational formulation, the resulting formulation is the equidistribution law. With this method, the resulting grid is usually postprocessed for the smoothness and the orthogonality. Due to its simplicity, the equidistribution law has been widely used as an adaptive grid generator (Refs. 52 - 58). So far, a completely automatic and robust adaptive grid generation method does not exist. It needs numerical experiments for 
adjusting the various constants, and often smoothness of the grid depends on the total number of grid points.

For the supersonic injection problem, the wave patterns produced by the system are very complex, and viscous and inviscid flow interaction is strong and complicated. To resolve all the important flow phenomena and to capture the generated waves, a numerical method must be robust and have a capability to capture all the existing discontinuities in the flow field clearly.

The objective of the present study is to combine the adaptive grid method with the upwind method and apply this combined procedure to the supersonic slot injection problem. To test the numerical method without any complications and uncertainties due to the turbulence modelling problem, the chosen sample test cases were 2-D, compressible, laminar flow cases. All the computational results are compared with exact solutions or experimental data. For some sample 2-D cases, the numerical accuracy of Flux Vector Splitting (FVS) based on van Leer's method and Flux Difference Splitting (FDS) based on Roe's approximate Riemann Solver are compared. Finally, FDS with the adaptive grid method is applied to the supersonic slot injection problems.

In Chap.2, the governing equations and the basic concepts of the adaptive grid method are discussed. Space discretization of the numerical method is presented in Chap. 3 along with time integration of the numerical methods. Detailed derivations of the linearization procedure necessary for the both FDS and FVS 
are given in the Chap. 4. Finally, the results of the numerical methods are compared with the experimental data and discussed. 


\subsection{Formulation}

\subsection{Governing Equations}

The unsteady, compressible, 2 - D, Navier - Stokes Equations can be expressed in a generalized coordinate system as

$$
\frac{1}{J} \frac{\partial Q}{\partial t}+\frac{\partial \hat{F}}{\partial \xi}+\frac{\partial \hat{G}}{\partial \eta}=\frac{1}{R e}\left[\frac{\partial \hat{F}_{v}}{\partial \xi}+\frac{\partial \hat{G}_{v}}{\partial \eta}\right]
$$

where

$$
\begin{array}{ll}
\hat{F}=\frac{1}{J}\left(\xi_{x} F+\xi_{y} G\right) & \hat{G}=\frac{1}{J}\left(\eta_{x} F+\eta_{y} G\right) \\
\hat{F}_{v}=\frac{1}{J}\left(\xi_{x} F_{v}+\xi_{y} G_{v}\right) & \hat{G}_{v}=\frac{1}{J}\left(\eta_{x} F_{v}+\eta_{y} G_{v}\right)
\end{array}
$$




$$
\begin{aligned}
& Q=[\rho, \rho u, \rho v, e]^{T} \\
& F=\left[\rho u, \rho u^{2}+p, \rho u v,(e+p) u\right]^{T} \\
& G=\left[\rho v, \rho u v, \rho v^{2}+p,(e+p) v\right]^{T} \\
& F_{v}=\left[0, \tau_{x x}, \tau_{x y}, s_{x}\right]^{T} \quad G_{v}=\left[0, \tau_{x y}, \tau_{y y}, s_{y}\right]^{T}
\end{aligned}
$$

The viscous and heat flux vectors are defined by

$$
\begin{aligned}
& \tau_{x x}=(\lambda+2 \mu) \frac{\partial u}{\partial x}+\lambda \frac{\partial v}{\partial y} \\
& \tau_{y y}=(\lambda+2 \mu) \frac{\partial v}{\partial y}+\lambda \frac{\partial u}{\partial x} \\
& \tau_{x y}=\mu\left(\frac{\partial u}{\partial y}+\frac{\partial v}{\partial x}\right) \\
& s_{x}=u \tau_{x x}+v \tau_{x y}+\frac{\mu}{(y-1) \operatorname{Pr} M_{\infty}^{2}} \frac{\partial T}{\partial x} \\
& s_{y}=u \tau_{x y}+v \tau_{y y}+\frac{\mu}{(y-1) \operatorname{Pr} M_{\infty}^{2}} \frac{\partial T}{\partial y}
\end{aligned}
$$

$\xi$ and $\eta$ in eq. (1) are a body coordinate system, and $J$ is the Jacobian of the transformation given by 


$$
\begin{aligned}
J & =\frac{\partial(\xi, \eta)}{\partial(x, y)} \\
& =\xi_{x} \eta_{y}-\eta_{x} \xi_{y} \\
& =\left(x_{\xi} y_{\eta}-x_{\eta} y_{\xi}\right)^{-1}
\end{aligned}
$$

The density, $\rho$, is nondimensionalized by its free stream value $\rho_{\infty}$, the velocities by $u_{\infty}$, the temperature by $T_{\infty}$, the total energy per unit volume e and pressure p by $\rho u_{\infty}^{2}$, the coordinates by a reference length $L$, the time $t$ by $\frac{L}{u_{\infty}}$, and the viscosity $\mu$ by $\mu_{\infty}$. The system is closed using the perfect gas equation of state, which can be written in nondimensional form as

$$
\begin{aligned}
& p=(\gamma-1)\left[e-\frac{\rho}{2}\left(u^{2}+v^{2}\right)\right] \\
& T=\gamma M_{\infty}^{2} \frac{p}{\rho}
\end{aligned}
$$

The viscosity $\mu$ is determined by the Sutherland formula and Stokes hypothesis, and the assumption of a constant Prandtl number is used to determine the conductivity of the gas, i.e. $\lambda=-\frac{2}{3} \mu, \mu=T^{\frac{3}{2}}\left(\frac{1+C}{T+C}\right)$, where the constant $\mathrm{C}$ is given as $C=\frac{110.4^{\circ} \mathrm{K}}{T_{\infty}}$. 


\subsection{Adaptive Grid}

The importance of grid generation in computational fluid dynamics is now well known. There has been rapid progress in the grid generation field, both in the conventional grid generation and in the adaptive grid generation method (Refs. $40-41,46)$. The accuracy of most numerical solution methods strongly depends upon the adequacy of the computational grid. It is not rare that the cause of poor or anomalous results of numerical methods can be attributed to an inadequate grid (Ref. 42).

Although various classifications of grid generation are possible, with respect to the interaction of grid generation and a numerical solution algorithm, we can classify grid generation into two main categories. One is the conventional grid generation method such as complex variable methods, algebraic methods and differential equation techniques (Ref. 42). Here, "conventional " means that grid points in the computational domain are distributed without any direct relation to the numerical solution except human experience. Contrary to the conventional grid generation method, the basic concept of adaptive grid generation is that the grid points in the computational domain are distributed such as to concentrate points in regions of large solution variation without a prior knowledge of the existence or location of such variations. 
If we further categorize the adaptive grid method in its implementation with the solution algorithm, there exist two different approaches. One is to consider the grid points as a function of time and include the time derivative terms of the grid points in the governing equations. Then,there is no need for interpolation. This approach is generally most suitable for unsteady problems. The other is to redistribute the grid at a specified time step and interpolate the solution from the old grid to a new grid. This can be regarded as a sequence of initial boundary value problems and is adequate for steady state problems (Ref. 56).

Another interesting aspect of the adaptive grid method is shock alignment. In an earlier paper, MacCormack and Paullay (Ref. 48) had shown that, if the grid is aligned with the shock, there are no overshoots and undershoots near the shock even if they used a central difference scheme. Recently, Davis (Ref. 49) applied FVS to solve an inviscid shock reflection problem defining the local direction around an oblique shock from the knowledge of the velocity gradients, and he showed that shock capturing capability can be greatly enhanced.

The final objective of any adaptive grid method is straightforward - to generate a good grid for the problem under consideration. Although there are some ambiguities in the definition of a good grid, the essential elements are smoothness, orthogonality and grid resolution adequate to the problem (Ref. 50). Among the several adaptive grid methods, the variational procedure of Brackbill and Saltzman (Ref. 51) is mathematically sound and elegant. They defined the measure of grid smoothness, orthogonalty and volume measure based on the 
some weighting function by suitable integration. In other words, they quantitized the quality of a grid and obtained a "good grid " by minimizing the resulting integrals. Despite the soundness of their method, this procedure is complex and time-consuming especially for 3-D problems, because the resulting equations are elliptic.

In the adaptive grid method such as the variational formulation method, there is a fixed number of grid points, and grid points are redistributed according to the solution variation. Another approach involves locally embedding sub-grids in regions of interest. Dannenhoffer and Baron (Ref. 52) used this method and obtained promising results for the inviscid flow problems. In this study, the grid point redistribution method was chosen because of its simplicity.

If grid smoothness and orthogonality are not included in the variational approach, we can get the so-called equidistribution law. Because of its simplicity, adaptive grid methods based on the equidistribution concept have been widely used (Refs. 53 - 59). Based on studies of numerical solutions of ordinary differential equations (Ref. 42), the total error can be reduced when the grid points are distributed by following relation

$$
\int_{x_{i}}^{x_{i+1}} w(x) d x=\text { const. }
$$


where $w(x)$ is a positive weighting function which should be related to the numerical error. This relation is known as an equidistribution law. The best choice for $w(x)$ is obviously the truncation error. But, for complex flow problems such as with the Navier-Stokes equations, generally it is difficult to derive the truncation error for the numerical scheme. Even if one knows the exact form of truncation error, the numerically calculated truncation error will be noisy and usually needs a complicated smoothing procedure, because the truncation error involves higher order derivatives.

As shown by Berger (Refs. $60-61$ ), use of Richardson's extrapolation with local mesh refinements can be a good method for evaluating the truncation errors. But, Richardson's extrapolation for evaluating the truncation error is only valid for smooth flow without any discontinuities in the computational domain. If one can find a function of a flow variable which is somehow related to the truncation error, it can be a substitute for the truncation error.

A widely used weighting function for the flow field calculation is the gradient of a flow variable. Sometimes second derivatives can be included. It must be pointed out that the choice of actual weighting function is problem dependent.

In the following, a brief explanation of the 1-D equidistribution law is given. To maintain a uniform grid in the computational space, combining the relation that $\Delta x=x_{\xi} \Delta \xi, \Delta \xi=1$ and eq. (2) yields the following relation.

$$
x_{\xi} w(\xi)=\text { const }
$$


Actually, eq. (3) is the Euler equation for minimizing the integral

$$
I=\int_{0}^{1} w(\xi) x_{\xi}^{2} d \xi
$$

Minimizing the integral is analogous to minimizing the potential energy of a system of springs with spring constants $w(\xi)$ and $\Delta x_{i}$ given by

$$
\Delta x_{i}=\frac{1}{w(\xi) N \int_{0}^{1} \frac{d \xi}{w(\xi)}}
$$

Besides, the spring analogy mentioned above, the so-called smoothness method is widely used for grid adaptation. The difference between the two is basically the difference of considering $w$ as a function of $x$ or as a function of $\xi$. These differences are explained in detail by Thompson (Ref. 42). When applying the equidistribution law to multidimensional problems, there should be some means which control the the grid smoothness and orthogonality. These are necessary, since using the equidistribution law, grids are generated without any relation to the neighboring points. In a novel paper by Nakahashi and Deiwert (Ref. 57), a torsional spring method for controlling the grid orthogonality and smoothness is proposed. The equation for the spring system can be written as

$$
w_{i j}\left(s_{i+1, j}-s_{i j}\right)-w_{i-1, j}\left(s_{i j}-s_{i-1, j}\right)-c \theta_{i, j-1}=0
$$


where $c=\lambda\left(\frac{1}{N} \sum_{k=1}^{N} w_{k J}\right), \theta=$ reference angle for torsional spring and $\lambda$ is a user specified constant. This equation is a tridiagonal system along the $\xi$ direction which can be easily solved. To control the minimum and maximum grid spacing, the weighting function used by Nakahashi and Deiwert has two constants such as

$$
w_{i}=1+A \bar{f}_{i}^{B}
$$

where $A$ and $B$ are positive constants which will be determined from the constraints of minimum and maximum grid spacing and $\bar{f}$ is a normalized function related to the weighting function. If the user specifies $\Delta x_{\max }, \Delta x_{\min }$ as input variables, it can be shown that

$$
A=\frac{\Delta x_{\max }}{\Delta x_{\min }}-1
$$

and B can be determined from the constraint of user specified minimum spacing, $\Delta x_{\min }$, by iteration.

For the inviscid case, a weighting function can be either $\nabla p$ or $\nabla \rho$, and any variable which can detect the shock works quite well. But, for viscous flow problems, besides capturing the shock, detecting the shear layer is also required.

For the supersonic slot injection problems here, a linear combination of the first and second derivative of $u$ is used in the $\mathrm{y}$ - direction. For the $\mathrm{x}$ - direction, a linear combination of the first and second derivative of $p$ is used. The second 
derivative of velocity is included in the weight function, because if the flow separates, the velocity gradient alone is not sufficient to detect the boundary layer correctly. 


\subsection{Space Discretization}

\subsection{Finite Volume Formulation}

The governing equations in eq. (1) are expressed in differential form. These equations can be also expressed as integral relations as

$$
\frac{\partial}{\partial t} \iint_{S} Q d x d y+\int_{\partial s} \vec{F} \cdot \hat{n} d s=0
$$

where $\vec{F}=\left(F-R e^{-1} F_{v}\right) \hat{i}+\left(G-R e^{-1} G_{v}\right) \hat{j}$. Applying this relation to a single cell element shown Fig. 2, and using the mid-point rule, it can be discretized as

$$
\begin{aligned}
\frac{1}{J} \frac{\partial Q}{\partial t} & +\left[\hat{F}-R e^{-1} \hat{F}_{v}\right]_{j+1 / 2, k}-\left[\hat{F}-R e^{-1} \hat{F}_{v}\right]_{j-1 / 2, k} \\
& +\left[\hat{G}-R e^{-1} \hat{G}_{v}\right]_{j, k+1 / 2}-\left[\hat{G}-\operatorname{Re}^{-1} \hat{G}_{v}\right]_{j, k-1 / 2}=0
\end{aligned}
$$


In eq. (10), $Q$ must be interpreted as a cell-averaged value. It must be pointed out that eq. (10) is not the only way to discretize the eq. (9). Other discretization approaches such as a cell-vertex scheme (Refs. 62 - 64) have their own advantages. Setting $\Delta \xi=\Delta \eta=1$, then $\frac{1}{J}$ can be interpreted as a cell area and length of cell interface; $\Delta S$ is calculated by

$$
\begin{aligned}
\Delta S_{j+1 / 2, k} & =\left[\frac{|\nabla \xi|}{J}\right]_{j+1 / 2, k} \\
\Delta S_{j, k+1 / 2} & =\left[\frac{|\nabla \eta|}{J}\right]_{j, k+1 / 2}
\end{aligned}
$$

Other terms are defined in eq. (1). This relation is called semi-discrete, since the time variable remains continuous. In a finite difference formulation with a generalized coordinate system, careful evaluation of the metric terms is required to get the uniform flow as an exact solution of the difference equations. With the finite volume formulation, uniform flow is an exact solution of the difference equations.

Currently, instead of using the full Navier-Stokes equations, thin-layer approximations of Navier-Stokes equations have been widely used in the numerical simulation of viscous flows. The accuracy of thin-layer approximations has been tested for various flow conditions including transonic airfoil flows by Visbal and Shang (Ref. 65), hypersonic separated flow over an indented nosetip (Ref. 66), and supersonic separated flow over on 20 degree wedge by Degani and Steger (Ref. 67) to name a few. In the thin-layer approximation to the 
Navier-Stokes equations, the viscous terms containing derivatives in the direction parallel to the body surfaces are neglected, i.e.

$$
\begin{aligned}
& \hat{F}_{v}=\frac{1}{J}\left(\xi_{x} F_{v}+\xi_{y} G_{v}\right)=\hat{F}_{v}^{\xi}\left(Q, Q_{\xi}\right)+\hat{F}_{v}^{\eta}\left(Q, Q_{\eta}\right) \\
& \hat{G}_{v}=\frac{1}{J}\left(\eta_{x} F_{v}+\eta_{y} G_{v}\right)=\hat{G}_{v}^{\xi}\left(Q, Q_{\xi}\right)+\hat{G}_{v}^{\eta}\left(Q, Q_{\eta}\right)
\end{aligned}
$$

For the thin-layer approximation, $\hat{F}_{v}^{\xi}, \hat{F}_{v}^{\eta}$, and $\hat{G}_{v}^{\xi}$ terms are neglected, and only the $\hat{G}_{v}^{\eta}$ term is retained.

The concept of the thin-layer approximation stems from the fact that adequate grid resolution both normal and parallel to the body surface is not feasible with current computers. As shown by MacCormack (Ref. 11), if $R e_{L}>10^{3}$, it is generally impractical to resolve the viscous $\mathrm{x}$-derivative terms of the Navier-Stokes equations. That means that even if the viscous terms in the $\mathrm{x}$-direction are retained, these terms will not be adequately resolved. The thin-layer approximation to the Navier-Stokes equations is valid for the numerical simulation of high Reynolds number flow if the flow does not separate massively (Ref. 67) . It also requires the body-aligned coordinate system. In this study, the thin-layer approximation was used to increase the computational efficiency. 


\subsection{Overview of upwind method}

In recent years, there have been great advances in the general upwind method. The idea of an upwind method is to discretize the hyperbolic differential equations by using differences based on the direction determined by the sign of the characteristic speed. The advantages of an upwind method are robustness, soundness of an underlying physical model, and the possibility of achieving high resolution of stationary discontinuities without oscillation. When solving the thin-layer Navier-Stokes equations based on the upwind method, the governing equations are considered as composed of inviscid and viscous terms. The inviscid terms are discretized based on the upwind method, and the viscous terms are discretized by standard central differencing. A brief discussion of the upwind method, which follows from the excellent description of Harten et al (Ref. 23), is given below.

Most of the current upwind methods can be thought as an extension of the Courant - Isaacson - Rees scheme (CIR scheme) to nonlinear conservation laws. The CIR scheme can be clarified by considering the constant coefficient scalar equation as

$$
u_{t}+a u_{x}=0 \quad a=\text { const. }
$$


The CIR scheme can be expressed as approximating $u_{x}$ as a backward difference if $a$ is positive and approximating $u_{x}$ as a forward difference if a is negative. The above statements can be expressed in more compact difference form as

$$
\begin{aligned}
u_{j}^{n+1} & =\lambda a^{+} u_{j-1}^{n}+\lambda(1-|a|) u_{j}^{n}-\lambda a^{-} u_{j+1}^{n} \\
& =u_{j}^{n}-\frac{\lambda}{2} a\left(u_{j+1}^{n}-u_{j-1}^{n}\right)+\frac{\lambda}{2}|a|\left(u_{j+1}^{n}-2 u_{j}^{n}+u_{j-1}^{n}\right)
\end{aligned}
$$

where $\lambda=\frac{\Delta t}{\Delta x}, a^{-}=\frac{1}{2}(a-|a|)$ and $a^{+}=\frac{1}{2}(a+|a|)$

The extension of the CIR scheme to nonlinear scalar conservation laws can be derived in a similar way (Ref.23).

For systems of hyperbolic conservation laws

$$
U_{t}+F(U)_{x}=0 \quad U(x, 0)=U_{0}(x)
$$

Here, $U(x, t)$ is a column vector of $\mathrm{m}$ unknowns and $F(U)$ is a vector of $\mathrm{m}$ components. To investigate the mathematical properties of eq. (15), it is better to transform eq. (15) in nonconservative form as

$$
U_{t}+A U_{x}=0
$$

where $A$ is the Jacobian matrix of $F(U)$. If eq. (16) is hyperbolic, then all the eigenvalues of the Jacobian matrix are real. In the constant coefficient case, $\mathrm{A}$ is constant and there exist characteristic variables $W=T^{-1} U$, which can diagonalize the system of equations. 


$$
W_{t}+\Lambda W_{x}=0
$$

where $\Lambda=T^{-1} A T$, and $\mathrm{T}$ is a matrix whose column is an eigenvector of Jacobian matrix A.

With the CIR scheme, eq. (17) can be approximated as

$$
W_{j}^{n+1}=W_{j}^{n}-\frac{\lambda}{2} \Lambda\left(W_{j+1}^{n}-W_{j-1}^{n}\right)+\frac{\lambda}{2}|\Lambda|\left(W_{j+1}^{n}-2 W_{j}^{n}+W_{j-1}^{n}\right)
$$

and in the original variable eq. (18) becomes

$$
U_{j}^{n+1}=U_{j}^{n}-\frac{\lambda}{2} A\left(U_{j+1}^{n}-U_{j-1}^{n}\right)+\frac{\lambda}{2}|A|\left(U_{j+1}^{n}-2 U_{j}^{n}+U_{j-1}^{n}\right)
$$

where $|A|=T|\Lambda| T^{-1}$. The situation is more complex for systems of nonlinear conservation laws such as Euler equations. There are currently two approaches for those equations. One is flux vector splitting (FVS) and the other is flux difference splitting (FDS). 


\subsection{Flux Vector Splitting}

Flux vector splitting of Steger and Warming (Ref. 20) is based on the fact that the flux vector $F$ is a homogeneous function of degree one in $Q$ for Euler equations. The flux vector can be expressed as

$$
F=A Q=\frac{\partial F}{\partial Q} Q
$$

Using a similarity transformation, eq. (20) becomes

$$
F=T \Lambda T^{-1} Q
$$

where $\Lambda$ is a diagonal matrix of eigenvalues, and $T$ is a matrix whose columns are the eigenvectors of $\mathrm{A}$. The diagonal matrix $\Lambda$ can be split as

$$
\Lambda=\Lambda^{+}+\Lambda^{-}
$$

where $\Lambda^{+}=\frac{\Lambda+|\Lambda|}{2}$ and $\Lambda^{-}=\frac{\Lambda-|\Lambda|}{2}$

Eq. (22) can be rewritten as

$$
\begin{aligned}
F & =T \Lambda T^{-1} Q=T\left(\Lambda^{+}+\Lambda^{-}\right) T^{-1} Q \\
& =\left(A^{+}+A^{-}\right) Q \\
& =F^{+}+F^{-}
\end{aligned}
$$

and the spatial derivatives of $F$ can be obtained as 


$$
\frac{\partial F}{\partial x}=\frac{\partial F^{+}}{\partial x}+\frac{\partial F^{-}}{\partial x}
$$

Here $\frac{\partial F^{+}}{\partial x}$ and $\frac{\partial F^{-}}{\partial x}$ are approximated by backward and forward difference operators, respectively. Although started from different backgrounds, there is a close relation between the central difference scheme and the upwind method. If one is using second order spatial discretization, Pulliam (Ref. 68) showed that

$$
\begin{aligned}
\frac{\partial F}{\partial x} & =\frac{\partial F^{+}}{\partial x}+\frac{\partial F^{-}}{\partial x} \\
& =\frac{1}{2}\left[\frac{\left(3 F_{j}^{+}-4 F_{j-1}^{+}+F_{j-2}^{+}\right)}{\Delta x}+\frac{\left(-3 F_{j}^{-}+4 F_{j+1}^{-}-F_{j+2}^{-}\right)}{\Delta x}\right]+O\left(\Delta x^{2}\right) \\
& =\bar{\delta}_{x} F+\frac{1}{4 \Delta x}\left(\Delta_{x} \nabla_{x}\right)^{2}|A| Q+\mathrm{O}\left(\Delta x^{2}\right)
\end{aligned}
$$

where $\bar{\delta}_{x} F=\frac{1}{4 \Delta x}\left(-F_{j+2}+4 F_{j+1}-4 F_{j-1}+F_{j-2}\right)$. This is just a second order central difference with numerical dissipation. This means that if artificial viscosity is carefully designed, it is possible to make the central difference scheme similar to the upwind method.

Despite the mathematical simplicity of Steger-Warming flux splitting, it has a drawback, namely the split fluxes are not differentiable at points where the sign of the eigenvalues changes, such as a stagnation point and sonic point. Recently, van Leer (Ref. 21) proposed a new flux vector splitting method with smooth transition where eigenvalues change sign. In this study, the flux vector splitting procedure developed by Anderson et al (Ref. 31) for the generalized coordinate system based on the Mach number, $\bar{M}$, normal to the cell interface is followed. 
The formulation for $\hat{F}^{ \pm}$in two dimensions is given in terms of the Mach number for supersonic flow $|\bar{M}| \geq 1$

$$
\hat{F}^{+}=\hat{F} \quad \hat{F}^{-}=0 \quad \bar{M} \geq 1
$$

$\hat{F}^{+}=0 \quad \hat{F}^{-}=\hat{F} \quad \bar{M} \leq-1$

and for subsonic flow, $|\bar{M}| \leq 1$

$$
\begin{aligned}
& \hat{F}^{ \pm}=\left[\begin{array}{c}
f_{1}^{ \pm} \\
f_{1}^{ \pm}\left[n_{x} \frac{(-\bar{u} \pm 2 c)}{\gamma}+u\right] \\
f_{1}^{ \pm}\left[n_{y} \frac{(-\bar{u} \pm 2 c)}{\gamma}+v\right] \\
f_{1}^{ \pm}\left[\frac{1}{\left(\gamma^{2}-1\right)}\left\{(\gamma-1) \bar{u}(-\bar{u} \pm 2 c)+2 c^{2}\right\}+\frac{q^{2}}{2}\right]
\end{array}\right] \\
& =\left[\begin{array}{lllll}
f_{1}^{ \pm} & f_{2}^{ \pm} & f_{3}^{ \pm} & f_{4}^{ \pm}
\end{array}\right]^{T}
\end{aligned}
$$

where

$$
\begin{aligned}
& f_{\overline{1}}^{ \pm}= \pm \frac{\rho}{4}[\bar{M} \pm 1]^{2} \frac{|\nabla \xi|}{J} \\
& \bar{u}=n_{x} u+n_{y} v \\
& \bar{M}=\frac{\bar{u}}{c}
\end{aligned}
$$


and $n_{x}, n_{y}$ are $\mathrm{x}, \mathrm{y}-$ components of the unit normal vector to a $\xi=$ constant cell interface.

The order of spatial accuracy for the numerical method is determined by the method of discretizing $\frac{\partial F^{+}}{\partial x}$ and $\frac{\partial F^{-}}{\partial x}$. If a higher order method is needed, there are two possibilities. One is simply to use higher order backward and forward differencing for $\frac{\partial F^{+}}{\partial x}$ and $\frac{\partial F^{-}}{\partial x}$. The other approach is so called the MUSCL (Monotonic Upstream-centered Scheme for Conservation Laws : code name) approach (Refs. 31 - 32) where one extrapolates $Q$ toward the cell interface and obtains $F^{+}$and $F^{-}$at the cell interface in the following way.

$$
\hat{F}(Q)_{j+1 / 2}=\hat{F}\left(Q^{-}, Q^{+}\right)_{j+1 / 2}=\hat{F}^{+}\left(Q^{-}\right)_{j+1 / 2}+\hat{F}^{-}\left(Q^{+}\right)_{j+1 / 2}
$$

where the subscript $\mathrm{k}$ is suppressed for simplicity. The notation $Q^{+}$and $Q^{-}$ implies that the dependent variables at the cell boundary are evaluated from the left and right respectively. $Q^{+}$and $Q^{-}$are computed by the following formula (Ref. 35)

$$
\begin{aligned}
\left(Q^{-}\right)_{j+1 / 2} & =Q_{j}+\frac{\phi}{4}[(1-\kappa) \nabla+(1+\kappa) \Delta] Q_{j} \\
& =\beta Q_{j}+\alpha Q_{j-1}+\gamma Q_{j+1} \\
\left(Q^{+}\right)_{j+1 / 2}= & Q_{j+1}-\frac{\phi}{4}[(1-\kappa) \Delta+(1+\kappa) \nabla] Q_{j+1} \\
= & \beta Q_{j+1}+\alpha Q_{j+2}+\gamma Q_{j}
\end{aligned}
$$


where $\nabla$ and $\Delta$ denote a backward difference operator and a forward difference operator, respectively, and $\alpha=-\frac{\phi}{4}(1-\kappa), \beta=1-\frac{\phi}{2} \kappa$ and $\gamma=\frac{\phi}{4}(1+\kappa)$. Thomas and Walters (Ref. 35) showed that for the one-dimensional case with uniform grid spacing, $\phi=0$ leads to 1st order accuracy. With $\phi=1$ and $\kappa=-1$, the 2 nd order fully upwind method results. The 3 rd order upwind biased approximation can be obtained with $\kappa=\frac{1}{3}$.

The MUSCL type approach was chosen here, since split fluxes are generally less differentiable than the conservation variables (Ref. 29). Also, if a generalized coordinate system is used, there are some ambiguities in choosing the correct metric terms at the cell interface which has sometimes degraded the accuracy of numerical results (Ref. 69).

The higher order approximation formula given in eqs. (27) and (28) can include a limiter which can suppress overshoots or undershoots near a discontinuity. If a limiter is activated at some points, the accuracy of the numerical method is reduced to 1st order at those points. Our experience with both a min-mod limiter and a continuously differentiable limiter (Ref. 31) for the shock-laminar boundary layer interaction problems showed that using the limiters gave worse results. For these reasons, a so-called " catastrophic limiter " (Ref. 32) was employed. With this limiter, if the higher order approximation results in negative values of pressure and density at some points, the 1st order approximation is used for those points. It turns out that with a non-adaptive grid, the catastrophic limiter was not activated for the problems studied here. 


\subsection{Flux Difference Splitting}

The basic concept of flux difference splitting is based on the solutions of the Riemann problem. The numerical flux at the cell interface is calculated from some exact or approximate solutions to the Riemann problem.

Here, Roe's approximate Riemann solver (Ref. 22) is adapted because this scheme is simple to use and shows good shock resolution in one dimensional cases. Roe's scheme can be clarified by considering the following linearized equation.

$$
U_{t}+A\left(U_{L}, U_{R}\right) U_{x}=0
$$

Roe proposed the following criteria to select a mean value of the Jacobian $A(U)$ of $F(U)$, i.e.

- $A(U, U)=A(U)$

- $A\left(U_{L} U_{R}\right)$ has a complete set of real eigenvalues and eigenvectors.

- $A\left(U_{L}, U_{R}\right)\left(U_{R}-U_{L}\right)=F\left(U_{R}\right)-F\left(U_{L}\right)$

The numerical flux at the cell interface based on Roe's method is expressed as

$$
F\left(U_{L}, U_{R}\right)=\frac{1}{2}\left[F\left(U_{L}\right)+F\left(U_{R}\right)-|\Delta F|\right]
$$


where the flux difference at the cell interface is defined as

$$
\begin{aligned}
|\Delta F| & =\left|A\left(U_{L}, U_{R}\right)\right|\left(U_{R}-U_{L}\right) \\
& =R|\Lambda| L\left(U_{R}-U_{L}\right)
\end{aligned}
$$

Here $|\Lambda|$ implies the absolute values of the elements of $\Lambda$, which are the eigenvalues of the Jacobian matrix $A . \mathrm{R}$ is the matrix with the right eigenvectors of Jacobian matrix $\mathrm{A}$ as columns, and $\mathrm{L}$ the matrix with left eigenvectors as rows.

Application of Roe's method to a 2-D problem is done by 1-D operator splitting. In this approach, two-dimensional wave interactions are approximated by combinations of one-dimensional wave interactions. This can be achieved in a straightfoward manner using the property of rotational invariance of the Euler equations (Ref. 70), i.e. :

$$
\hat{F}=\frac{|\nabla \xi|}{J}\left[n_{x} F(Q)+n_{y} G(Q)\right]=\frac{|\nabla \xi|}{J} T^{-1} F(\bar{Q})
$$

where $\frac{|\nabla \xi|}{J}$ is cell face length, $\mathrm{T}$ is the transformation matrix given as

$$
T=\left[\begin{array}{cccc}
1 & 0 & 0 & 0 \\
0 & n_{x} & n_{y} & 0 \\
0 & -n_{y} & n_{x} & 0 \\
0 & 0 & 0 & 1
\end{array}\right]
$$

and $\bar{Q}=T Q$, which is expressed as 


$$
\bar{Q}=\left[\begin{array}{c}
\rho \\
\rho \bar{u} \\
\rho \bar{v} \\
e
\end{array}\right]
$$

where $\bar{u}$ and $\bar{v}$ are velocities normal and parallel to the cell interface, and Roe's method will be applied using the $\bar{u}$ same as in a 1-D case. Then, flux difference can be expressed as

$$
|\Delta \bar{F}|=\bar{R}|\bar{\Lambda}| \bar{L}\left(\bar{Q}_{R}-\bar{Q}_{L}\right)
$$

$|\Delta \hat{F}|=T^{-1} \bar{R}|\bar{\Lambda}| \bar{L} T\left(Q_{R}-Q_{L}\right)$

where $\bar{L}$ and $\bar{R}$ are defined as quantities whose rows and columns are left and right eigenvectors of Jacobian matrix $\frac{\partial \bar{F}}{\partial \bar{Q}}$ given by

$$
\begin{aligned}
\bar{R} & =\left[\begin{array}{cccc}
1 & 0 & 1 & 1 \\
\bar{u} & 0 & \bar{u}+c & \bar{u}-c \\
\bar{v} & 1 & \bar{v}+c & \bar{v}-c \\
\frac{\bar{u}^{2}+\bar{v}^{2}}{2} & \bar{v} & \bar{H}+\bar{u} c & \bar{H}-\bar{u} c
\end{array}\right] \\
\bar{L} & =\left[\begin{array}{cccc}
1-\frac{b}{2} q^{2} & b \bar{u} & b \bar{v} & -b \\
-\bar{v} & 0 & 1 & 0 \\
\frac{1}{2}\left(\frac{b}{2} q^{2}-\frac{\bar{u}}{c}\right) & \frac{1}{2}\left(-b \bar{u}+\frac{1}{c}\right) & -\frac{b}{2} \bar{v} & \frac{b}{2} \\
\frac{1}{2}\left(\frac{b}{2} q^{2}+\frac{\bar{u}}{c}\right) & \frac{1}{2}\left(-b \bar{u}-\frac{1}{c}\right) & -\frac{b}{2} \bar{v} & \frac{b}{2}
\end{array}\right]
\end{aligned}
$$


with $b=\frac{\gamma-1}{c^{2}}$

Defining $\tilde{M}=T^{-1} \bar{R}|\bar{\Lambda}| \bar{L} T$, then the numerical flux at the cell interface for the Euler equations can be evaluated by following manner

$$
\hat{F}_{j+1 / 2, k}=\frac{1}{2}\left[\hat{F}\left(Q_{j+1 / 2, k}^{R}\right)+\hat{F}\left(Q_{j+1 / 2, k}^{L}\right)-\tilde{M}_{j+1 / 2, k}\left(Q_{j+1 / 2, k}^{R}-Q_{j+1 / 2, k}^{L}\right)\right]
$$

Here $\tilde{M}_{j+1 /, k}$ is a Jacobian matrix based on a special averaging procedure, namely Roe's averaging which reads as

$$
\begin{aligned}
& \tilde{\rho}=\sqrt{\rho_{L} \rho_{R}} \\
& \tilde{u}=\frac{u_{R} \sqrt{\rho_{R}}+u_{L} \sqrt{\rho_{L}}}{\sqrt{\rho_{L}}+\sqrt{\rho_{R}}} \\
& \tilde{v}=\frac{v_{R} \sqrt{\rho_{R}}+v_{L} \sqrt{\rho_{L}}}{\sqrt{\rho_{L}}+\sqrt{\rho_{R}}} \\
& \tilde{H}=\frac{H_{R} \sqrt{\rho_{R}}+H_{L} \sqrt{\rho_{L}}}{\sqrt{\rho_{L}}+\sqrt{\rho_{R}}}
\end{aligned}
$$

and the speed of sound can be calculated by

$$
\tilde{c}^{2}=(\gamma-1)\left[\tilde{H}-\frac{1}{2}\left(\tilde{u}^{2}+\tilde{v}^{2}\right)\right]
$$


By using eqs. (32)-(35), the numerical flux difference at the cell interface based on Roe's approximate Riemann solver can be expressed in the following computationally efficient form (Ref. 27).

$\tilde{M} \Delta Q=|\Delta F|_{1}+|\Delta F|_{2}+|\Delta F|_{3}+|\Delta F|_{4}$

$|\Delta F|_{1}=\left|\Lambda_{1}\right|\left(\Delta \rho-\frac{\Delta p}{c^{2}}\right)\left[\begin{array}{c}1 \\ u \\ v \\ \frac{q^{2}}{2}\end{array}\right]$

$|\Delta F|_{2}=\left|\Lambda_{2}\right| \rho\left[\begin{array}{c}0 \\ \Delta u-n_{x} \Delta \bar{u} \\ \Delta v-n_{y} \Delta \bar{u} \\ \Delta\left(\frac{q^{2}}{2}\right)-\bar{u} \Delta \bar{u}\end{array}\right]$

$|\Delta F|_{3,4}=\left|\Lambda_{3,4}\right| \frac{\Delta p \pm \rho c \Delta \bar{u}}{2 c^{2}}\left[\begin{array}{c}1 \\ u \pm n_{x} c \\ v \pm n_{y} c \\ H \pm c \bar{u}\end{array}\right]$

Where $\Lambda$ are eigenvalues of the Jacobian matrix given by

$$
\begin{aligned}
& \Lambda_{1}=\bar{u} \frac{|\nabla \xi|}{J} \\
& \Lambda_{2}=\Lambda_{1}
\end{aligned}
$$


$\Lambda_{3}=(\bar{u}+c) \frac{|\nabla \xi|}{J}$

$\Lambda_{4}=(\bar{u}-c) \frac{|\nabla \xi|}{J}$

To increase the order of accuracy, $Q^{R}$ and $Q^{L}$ are computed by MUSCL extrapolation formulas. Here, $\mathrm{Q}$ can be either conservative, primitive or characteristic variables. Our numerical experimentations with the shock-laminar boundary layer interaction problem showed that the difference between using the conservative or primitive variables is small both in accuracy and convergence rate. In this study, primitive variables are used to calculate the slope, since using the conservative variables gave slightly worse results for some problems (Ref. 98).

Roe's method does not satisfy the entropy condition and thus permits stable expansion shocks for inviscid problems. For such a method, the numerical diffusion component of the numerical flux can vanish at a sonic rarefaction. Both expansion shocks and sonic glitches can be eliminated by using augmented numerical diffusion.

Harten (Ref. 25) proposed a simple entropy fix method such as

$$
|\Lambda|=\left[\begin{array}{cc}
|\Lambda| & \text { if }|\Lambda| \geq \varepsilon \\
\frac{\Lambda^{2}+\varepsilon^{2}}{2 \varepsilon} & \text { if }|\Lambda|<\varepsilon
\end{array}\right]
$$


where $\Lambda$ is the eigenvalues of Jacobian matrix based on Roe's averaging, and $\varepsilon$ is a small positive number. As explained by Harten, this kind of entropy fix applies only to discontinuities in a genuinely nonlinear field, since there are no entropy considerations in a linearly degenerate one. For the Euler equations, the characterstic field corresponding to the eigenvalue, $u$, is linearly degenerate, and the other characteristic fields corresponding to the eigenvalue $u \pm c$ are genuinely nonlinear.

\subsection{Treatment of Viscous terms}

The viscous terms in the thin-layer equations have only a component in the direction of $\eta$ and in finite volume formulation can be expressed as

$$
\begin{gathered}
\hat{G}_{v}=J\left(\frac{|\nabla \xi|}{J}\right)^{2} \mu\left[\begin{array}{c}
0 \\
\left(1+\frac{1}{3} t_{x}^{2}\right) u_{\eta}+\frac{1}{3} t_{x} t_{y} v_{\eta} \\
\left(1+\frac{1}{3} t_{y}^{2}\right) v_{\eta}+\frac{1}{3} t_{x} t_{y} u_{\eta} \\
\frac{1}{2}\left[\left(1+\frac{1}{3} t_{x}^{2}\right)\left(u^{2}\right)_{\eta}+\left(1+\frac{1}{3} t_{y}^{2}\right)\left(v^{2}\right)_{\eta}\right] \\
+\frac{1}{3} t_{x} t_{y}(u v)_{\eta}+\frac{1}{(\gamma-1) \operatorname{Pr} M^{2}} T_{\eta}
\end{array}\right] \\
=J\left(\frac{|\nabla \xi|}{J}\right)^{2} \mu \hat{T}
\end{gathered}
$$

Viscous terms are approximated by central differencing as 


$$
\begin{aligned}
{\left[\frac{\partial \hat{G}_{v}}{\partial \eta}\right]_{k} } & =\frac{\partial}{\partial \eta}\left[J\left(\frac{|\nabla \xi|}{J}\right)^{2} \mu \hat{T}\right]_{k} \\
& =\left[J\left(\frac{|\nabla \xi|}{J}\right)^{2} \mu \hat{T}\right]_{k+1 / 2}-\left[J\left(\frac{|\nabla \xi|}{J}\right)^{2} \mu \hat{T}\right]_{k-1 / 2}
\end{aligned}
$$

When evaluating $\frac{\partial \hat{G}_{v}}{\partial \eta}$, a cell area term at the cell interface is needed which is approximated by the average of nearby cell area values. At the wall, derivatives are computed by one-sided differencing. 


\subsection{Time Integration}

The space discretization procedures discussed in the last section lead to a set of coupled ordinary differential equations, which can be written in semi-discrete form as

$$
\frac{1}{J} \frac{d Q}{d t}+R(Q)=0
$$

where $R(Q)$ is the vector of residuals, and, at steady state, this term should be zero.

\subsection{Backward Euler Time Integration}

The time integration scheme adapted here for the thin-layer Navier- Stokes equations is backward Euler time integration and given by 


$$
\begin{aligned}
\Delta Q & =Q^{n+1}-Q^{n} \\
& =\left[\frac{\partial Q}{\partial t}\right]^{n+1} \Delta t+O\left(\Delta t^{2}\right)
\end{aligned}
$$

Backward Euler time integration is chosen because this scheme shows a rapid convergence rate with various relaxation methods. Although this integration is only 1st order in time, if one is interested in the steady state results that will not be a problem.

From eq. (1), the steady state residual is defined as

$$
\begin{aligned}
\frac{1}{J} \frac{\partial Q^{n}}{\partial t} & =-\frac{\partial \hat{F}^{n}}{\partial \xi}-\frac{\partial \hat{G}^{n}}{\partial \eta}+\frac{1}{R e}\left[\frac{\partial \hat{F}_{v}}{\partial \xi}+\frac{\partial \hat{G}_{v}}{\partial \eta}\right]^{n} \\
& =-R^{n}
\end{aligned}
$$

Using eq. (43) and dropping the truncation terms, eq. (42) becomes

$$
\frac{1}{J} \frac{\Delta Q}{\Delta t}=-\left[\frac{\partial \hat{F}}{\partial \xi}\right]^{n+1}-\left[\frac{\partial \hat{G}}{\partial \eta}\right]^{n+1}+\frac{1}{R e}\left[\frac{\partial \hat{F}_{v}}{\partial \xi}+\frac{\partial \hat{G}_{v}}{\partial \eta}\right]^{n+1}
$$

For the implicit scheme, $\hat{F}^{n+1}, \hat{G}^{n+1}$ and $\hat{G}_{v}^{n+1}$ are needed in a linearized form. These terms can be obtained by using a Taylor series expansion.

$$
\begin{aligned}
\hat{F}^{n+1} & =\hat{F}^{n}+\frac{\partial \hat{F}}{\partial Q}\left(Q^{n+1}-Q^{n}\right)+O\left(\Delta t^{2}\right) \\
& \simeq \hat{F}^{n}+\hat{A}^{n} \Delta Q \\
\hat{G}^{n+1} & \simeq \hat{G}^{n}+\hat{B}^{n} \Delta Q
\end{aligned}
$$


where $\hat{A}$ and $\hat{B}$ are the Jacobian matrices. When linearizing the viscous term in the thin-layer Navier-Stokes equations, since $\hat{G}_{v}=\hat{G}_{v}\left(Q, Q_{\eta}\right)$ and dropping the truncation error terms, one gets

$$
\begin{aligned}
\hat{G}_{v}^{n+1} & =\hat{G}_{v}^{n}+\frac{\partial \hat{G}_{v}}{\partial Q}\left(Q^{n+1}-Q^{n}\right)+\frac{\partial \hat{G}_{v}}{\partial Q_{\eta}}\left(Q_{\eta}^{n+1}-Q_{\eta}^{n}\right) \\
& =\hat{G}_{v}^{n}+\hat{H}_{1} \Delta Q+\hat{H}_{2} \Delta Q_{\eta}
\end{aligned}
$$

where $\hat{H}_{1}=\frac{\partial \hat{G}_{v}}{\partial Q}$ and $\hat{H}_{2}=\frac{\partial \hat{G}_{v}}{\partial Q_{\eta}}$. Rearranging the terms in eq. (45)

$$
\begin{aligned}
\hat{G}_{v}^{n+1} & =\hat{G}_{v}^{n}+\hat{H}_{1} \Delta Q+\frac{\partial}{\partial \eta}\left(\hat{H}_{2} \Delta Q\right)-\frac{\partial \hat{H}_{2}}{\partial \eta} \Delta Q \\
& =\hat{G}_{v}^{n}+\left(\hat{H}_{1}-\frac{\partial \hat{H}_{2}}{\partial \eta}\right) \Delta Q+\frac{\partial}{\partial \eta}\left(\hat{H}_{2} \Delta Q\right)
\end{aligned}
$$

As shown by Beam and Warming, if $\mu$ is assumed locally constant then eq. (46) reduces to

$$
\Delta \hat{G}_{v}=\hat{G}_{v}^{n+1}-\hat{G}_{v}^{n}=\frac{\partial}{\partial \eta}\left(\hat{H}_{2} \Delta Q\right)
$$

From eq. (44)

$$
\frac{I}{J} \frac{\Delta Q}{\Delta t}+\frac{\partial \Delta \hat{F}}{\partial \xi}+\frac{\partial \Delta \hat{G}}{\partial \eta}-\frac{1}{R e} \frac{\partial \Delta \hat{G}_{v}}{\partial \eta}=-\left[\frac{\partial \hat{F}}{\partial \xi}+\frac{\partial \hat{G}}{\partial \eta}-\frac{1}{R e} \frac{\partial \hat{G}_{v}}{\partial \eta}\right]^{n}
$$

here I is an identity matrix. The linearization given by eqs. (42), (46) with (47) results a linearized system in delta form as 
$\left[\frac{I}{J} \frac{\Delta Q}{\Delta t}+\frac{\partial}{\partial \xi}\left(\hat{A}^{n} \Delta Q\right)+\frac{\partial}{\partial \eta}\left(\hat{B}^{n} \Delta Q\right)-\frac{1}{R e} \frac{\partial^{2}}{\partial \eta^{2}}\left(\hat{H}_{2}^{n} \Delta Q\right)\right]=-R^{n}$

This can be expressed in more compact form as

$\left[\frac{I}{J \Delta t}+\frac{\partial}{\partial \xi}\left(\hat{A}^{n}\right)+\frac{\partial}{\partial \eta}\left(\hat{B}^{n}\right)-\frac{1}{R e} \frac{\partial^{2}}{\partial \eta^{2}}\left(\hat{H}_{2}^{n}\right)\right] \Delta Q=-R^{n}$

In the above, the right hand side is usually called the explicit part and left hand side is called the implicit part. 


\subsection{Jacobians}

With Backward Euler time integration, the system of equations can be written as

$$
\left[\frac{I}{J \Delta t}+\frac{\partial R}{\partial Q}\right]^{n} \Delta Q=-R^{n}
$$

It turns out that if $\Delta t \rightarrow \infty$, this schemes converts to the Newton method, and, if the evaluation of the Jacobian matrix is exact, fast convergence can be expected. Linearization will be discussed in two parts, linearization of the inviscid terms and linearization of the viscous terms. First, linearization of inviscid terms based on the upwind method will be discussed followed by a linearization of viscous terms based on the central differencing.

\subsubsection{Jacobians of Inviscid Terms}

For van Leer's Flux Vector Splitting, the residual is given as

$$
\begin{aligned}
R_{j k}^{n} & =\hat{F}^{+}\left(Q_{j+1 / 2, k}^{-}\right)+\hat{F}^{-}\left(Q_{j+1 / 2, k}^{+}\right)-\hat{F}^{+}\left(Q_{j-1 / 2, k}^{-}\right)-\hat{F}^{-}\left(Q_{j-1 / 2, k}^{+}\right) \\
& +\hat{G}^{+}\left(Q_{j, k+1 / 2}^{-}\right)+\hat{G}^{-}\left(Q_{j, k+1 / 2}^{+}\right)-\hat{G}^{+}\left(Q_{j, k-1 / 2}^{-}\right)-\hat{G}^{-}\left(Q_{j, k-1 / 2}^{+}\right) \\
& -\frac{1}{\operatorname{Re}_{L}}\left[\hat{G}_{\nu}\left(Q_{j, k+1 / 2}\right)-\hat{G}_{\nu}\left(Q_{j, k-1 / 2}\right)\right]
\end{aligned}
$$


With higher order extrapolation given in eq. (27) and eq. (28), $\frac{\partial R}{\partial Q}$ for the inviscid terms is calculated in the following way.

$$
\frac{\partial R_{j k}}{\partial Q_{j-2, k}}=-\alpha \hat{A}_{j-1 / 2, k}^{+}
$$

$\frac{\partial R_{j k}}{\partial Q_{j-1, k}}=\alpha \hat{A}_{j+1 / 2, k}^{+}-\beta \hat{A}_{j-1 / 2, k}^{+}-\gamma \hat{A}_{j-1 / 2, k}^{-}$

$\frac{\partial R_{j k}}{\partial Q_{j, k}}=\beta\left(\hat{A}_{j+1 / 2, k}^{+}-\hat{A}_{j-1 / 2, k}^{-}\right)+\gamma\left(\hat{A}_{j-1 / 2, k}^{-}-\hat{A}_{j-1 / 2, k}^{+}\right)$

$\frac{\partial R_{j k}}{\partial Q_{j+1, k}}=\gamma \hat{A}_{j+1 / 2, k}^{+}+\beta \hat{A}_{j+1 / 2, k}^{-}-\alpha \hat{A}_{j-1 / 2, k}^{-}$

$\frac{\partial R_{j k}}{\partial Q_{j+2, k}}=\alpha \hat{A}_{j+1 / 2, k}^{-}$

where $\hat{A}^{ \pm}=\frac{\partial \hat{F}^{ \pm}}{\partial Q}$, and the elements of this matrix $\left(=\hat{a}_{j k}\right)$ are given as

$$
\begin{aligned}
& \hat{a}_{11}^{ \pm}=\left[ \pm \frac{1}{4}\left(1-\bar{M}^{2}\right)\left[c+\frac{\gamma(\gamma-1)}{2 c}\left(q^{2}-\frac{e}{\rho}\right)\right]\right] \frac{|\nabla \xi|}{J} \\
& \hat{a}_{\overline{12}}^{ \pm}=\left[ \pm \frac{\gamma(1-\gamma)}{8 c}\left(1-\bar{M}^{2}\right) u+(1 \pm \bar{M}) \frac{n_{x}}{2}\right] \frac{|\nabla \xi|}{J}
\end{aligned}
$$




$$
\begin{aligned}
& \hat{a}_{13}^{ \pm}=\left[ \pm \frac{\gamma(1-\gamma)}{8 c}\left(1-\bar{M}^{2}\right) v+(1 \pm \bar{M}) \frac{n_{y}}{2}\right] \frac{|\nabla \xi|}{J} \\
& \hat{a}_{14}^{ \pm}=\left[ \pm \frac{\gamma(\gamma-1)}{8 c}\left(1-\bar{M}^{2}\right)\right] \frac{|\nabla \xi|}{J} \\
& \hat{a}_{21}^{ \pm}=\frac{f_{2}^{ \pm}}{f_{1}^{ \pm}} \hat{a}_{11}^{ \pm} \pm f_{1}^{ \pm} \frac{(\gamma-1) n_{x}}{\rho c}\left(q^{2}-\frac{e}{\rho}\right)+\frac{f_{1}^{ \pm}}{\gamma \rho}\left(n_{x} \bar{u}-\gamma u\right) \\
& \hat{a}_{22}^{ \pm}=\frac{f_{2}^{ \pm}}{f_{1}^{ \pm}} \hat{a}_{12}^{ \pm} \pm f_{1}^{ \pm} \frac{(1-\gamma) n_{x}}{\rho c} u-\frac{f_{1}^{ \pm}}{\gamma \rho}\left(n_{x}^{2}-\gamma\right) \\
& \hat{a}_{23}^{ \pm}=\frac{f_{2}^{ \pm}}{f_{1}^{ \pm}} \hat{a}_{13}^{ \pm} \pm f_{1}^{ \pm} \frac{(1-\gamma) n_{x}}{\rho c} \nu-\frac{f_{1}^{ \pm}}{\gamma \rho} n_{x} n_{y} \\
& \hat{a}_{24}^{ \pm}=\frac{f_{2}^{ \pm}}{f_{1}^{ \pm}} \hat{a}_{14}^{ \pm} \pm f_{1}^{ \pm} \frac{(\gamma-1) n_{x}}{\rho c} \\
& \hat{a}_{31}^{ \pm}=\frac{f_{3}^{ \pm}}{f_{1}^{ \pm}} \hat{a}_{11}^{ \pm} \pm f_{1}^{ \pm} \frac{(\gamma-1) n_{y}}{\rho c}\left(q^{2}-\frac{e}{\rho}\right)+\frac{f_{1}^{ \pm}}{\gamma \rho}\left(n_{y} \bar{u}-\gamma v\right) \\
& \hat{a}_{32}^{ \pm}=\frac{f_{3}^{ \pm}}{f_{1}^{ \pm}} \hat{a}_{12}^{ \pm} \pm f_{1}^{ \pm} \frac{(1-\gamma) n_{y}}{\rho c} u-\frac{f_{1}^{ \pm}}{\gamma \rho} n_{x} n_{y} \\
& \hat{a}_{33}^{ \pm}=\frac{f_{3}^{ \pm}}{f_{1}^{ \pm}} \hat{a}_{13}^{ \pm} \pm f_{1}^{ \pm} \frac{(1-\gamma) n_{y}}{\rho c} \nu-\frac{f_{1}^{ \pm}}{\gamma \rho}\left(n_{y}^{2}-\gamma\right)
\end{aligned}
$$


$\hat{a}_{41}^{ \pm}=\frac{f_{\frac{t}{4}}^{ \pm}}{f_{1}^{ \pm}} \hat{a}_{11}^{ \pm}+\frac{f_{1}^{ \pm}}{\rho}\left[\frac{\gamma}{\gamma+1}\{2 c \pm(\gamma-1) \bar{u}\} \frac{1}{c}\left(q^{2}-\frac{e}{\rho}\right)-\frac{2 \bar{u}}{\gamma+1}(-\bar{u} \pm c)-q^{2}\right]$

$\hat{a}_{42}^{ \pm}=\frac{f_{4}^{ \pm}}{f_{1}^{ \pm}} \hat{a}_{12}^{ \pm}+\frac{f_{1}^{ \pm}}{\rho}\left[-\frac{\gamma}{\gamma+1}\{2 c \pm(\gamma-1) \bar{u}\} \frac{u}{c}-\frac{2}{\gamma+1}(-\bar{u} \pm c) n_{x}+u\right]$

$\hat{a}_{43}^{ \pm}=\frac{f_{4}^{t}}{f_{1}^{ \pm}} \hat{a}_{\overline{1}}^{ \pm}+\frac{f_{1}^{ \pm}}{\rho}\left[-\frac{\gamma}{\gamma+1}\{2 c \pm(\gamma-1) \bar{u}\} \frac{v}{c}-\frac{2}{\gamma+1}(-\bar{u} \pm c) n_{y}+v\right]$

$\hat{a}_{44}^{ \pm}=\frac{f_{\frac{t}{4}}^{ \pm}}{f_{1}^{ \pm}} \hat{a}_{\overline{1}}^{ \pm}+\frac{f_{1}^{ \pm}}{\rho c}\left[\frac{\gamma}{\gamma+1}\{2 c \pm(\gamma-1) \bar{u}\}\right]$

The expressions for $\hat{B}^{ \pm}=\left[\frac{\partial \hat{G} \pm}{\partial Q}\right]_{j k}$ are obtained by replacing $n_{x}, n_{y}$ and $J^{-1}|\nabla \xi|$ by $t_{x}, t_{y}$ and $J-1|\nabla \eta|$, respectively.

As shown above, exact linearization is possible for van Leer's FVS scheme. With the above linearization, the whole matrix system obtained is a large banded block matrix with block size of four, and the total number of matrix element is $\left(4^{*}\right.$ $\left.j_{\max } * k_{\max }\right) \times\left(4^{*} j_{\max } * k_{\max }\right)$. If $j_{\max }=80$ and $k_{\max }=90$ then the resulting algebraic system is a $28800 \times 28800$ matrix problem. Because of the size of the matrix, directly solving eq. (51) is not efficient. When solving the system of equations, the vertical line Gauss-Seidel method was used. For the vertical line Gauss-Seidel method, the L-U decomposition part can be vectorized over the lines, but the back substitution part cannot be vectorized because of recurrence. 
For Roe's FDS method the residual is given as

$$
\begin{aligned}
R_{j k}^{n}= & \frac{1}{2}\left[\hat{F}\left(Q_{j+1 / 2, k}^{-}\right)+\hat{F}\left(Q_{j+1 / 2, k}^{+}\right)-\tilde{M}_{j+1 / 2, k}\left(Q_{j+1 / 2, k}^{+}-Q_{j+1 / 2, k}^{-}\right)\right] \\
- & \frac{1}{2}\left[\hat{F}\left(Q_{j-1 / 2, k}^{-}\right)+\hat{F}\left(Q_{j-1 / 2, k}^{+}\right)-\tilde{M}_{j-1 / 2, k}\left(Q_{j-1 / 2, k}^{+}-Q_{j-1 / 2, k}^{-}\right)\right] \\
+ & \frac{1}{2}\left[\hat{G}\left(Q_{j, k+1 / 2}^{-}\right)+\hat{G}\left(Q_{j, k+1 / 2}^{+}\right)-\tilde{M}_{j, k+1 / 2}\left(Q_{j, k+1 / 2}^{+}-Q_{j, k+1 / 2}^{-}\right)\right] \\
- & \frac{1}{2}\left[\hat{G}\left(Q_{j, k-1 / 2}^{-}\right)+\hat{G}\left(Q_{j, k-1 / 2}^{+}\right)-\tilde{M}_{j, k-1 / 2}\left(Q_{j, k-1 / 2}^{+}-Q_{j, k-1 / 2}^{-}\right)\right] \\
& -\frac{1}{\operatorname{Re}_{L}}\left[\hat{G}_{v}\left(Q_{j, k+1 / 2}\right)-\hat{G}_{v}\left(Q_{j, k-1 / 2}\right)\right]
\end{aligned}
$$

It turns out that with Roe's FDS method, exact linearization is impractical because of the term $\frac{\partial \tilde{M}}{\partial Q}$, which is difficult to obtain and needs a lot of operation counts for numerical implementation. In the following, we neglect the $\frac{\partial \tilde{M}}{\partial Q}$ term in the implicit terms. As shown by Barth (Ref. 71), this linearization leads to time conservative discretization and has significant advantages over the time nonconservative linearization.

Linearization of Roe's scheme for the inviscid terms is given by

$$
\begin{aligned}
\frac{\partial R_{j k}}{\partial Q_{j-2, k}} & =-\frac{\alpha}{2}\left(\hat{A}_{j-1 / 2, k}^{L}+\tilde{M}_{j-1 / 2, k}\right) \\
\frac{\partial R_{j k}}{\partial Q_{j-1, k}} & =\frac{\alpha}{2}\left(\hat{A}_{j+1 / 2, k}^{L}-\tilde{M}_{j+1 / 2, k}\right)-\frac{\beta}{2}\left(\hat{A}_{j-1 / 2, k}^{L}-\tilde{M}_{j-1 / 2, k}\right)-\frac{\gamma}{2}\left(\hat{A}_{j-1 / 2, k}^{R}-\tilde{M}_{j-1 / 2, k}\right) \\
\frac{\partial R_{j k}}{\partial Q_{j, k}} & =\frac{\beta}{2}\left(\hat{A}_{j+1 / 2, k}^{L}+\tilde{M}_{j+1 / 2, k}\right)+\frac{\gamma}{2}\left(\hat{A}_{j+1 / 2, k}^{R}-\tilde{M}_{j+1 / 2, k}\right) \\
& -\frac{\gamma}{2}\left(\hat{A}_{j-1 / 2, k}^{L}+\tilde{M}_{j-1 / 2, k}\right)-\frac{\beta}{2}\left(\hat{A}_{j-1 / 2, k}^{R}-\tilde{M}_{j-1 / 2, k}\right)
\end{aligned}
$$




$$
\begin{aligned}
& \frac{\partial R_{j k}}{\partial Q_{j+1, k}}=\frac{\gamma}{2}\left(\hat{A}_{j+1 / 2, k}^{L}+\tilde{M}_{j+1 / 2, k}\right)+\frac{\beta}{2}\left(\hat{A}_{j+1 / 2, k}^{R}-\tilde{M}_{j+1 / 2, k}\right)-\frac{\alpha}{2}\left(\hat{A}_{j-1 / 2, k}^{R}-\tilde{M}_{j-1 / 2, k}\right) \\
& \frac{\partial R_{j k}}{\partial Q_{j+2, k}}=\frac{\alpha}{2}\left(\hat{A}_{j+1 / 2, k}^{L}-\tilde{M}_{j+1 / 2, k}\right)
\end{aligned}
$$

where $\hat{A}^{L}=\frac{\partial F\left(Q^{-}\right)}{\partial Q}$ and $\hat{A}^{R}=\frac{\partial F\left(Q^{+}\right)}{\partial Q}$.

The elements of the unsplit Jacobian matrix,$\hat{a}_{j k}$, are given as follows

$$
\begin{aligned}
& \hat{a}_{11}=0 \quad \hat{a}_{12}=\hat{n}_{x} \quad \hat{a}_{13}=\hat{n}_{y} \quad \hat{a}_{14}=0 \\
& \hat{a}_{21}=-u \hat{U}+\hat{n}_{x} \frac{\gamma-1}{2}\left(u^{2}+v^{2}\right) \quad \hat{a}_{22}=(2-\gamma) u \hat{n}_{x}+\hat{U} \\
& \hat{a}_{23}=u \hat{n}_{y}-v \hat{n}_{x}(\gamma-1) \quad \hat{a}_{24}=(\gamma-1) \hat{n}_{x} \\
& \hat{a}_{31}=-v \hat{U}+\hat{n}_{y} \frac{\gamma-1}{2}\left(u^{2}+v^{2}\right) \quad \hat{a}_{32}=v \hat{n}_{x}-u \hat{n}_{y}(\gamma-1) \\
& \hat{a}_{33}=(2-\gamma) v \hat{n}_{y}+\hat{U} \quad \hat{a}_{34}=(\gamma-1) \hat{n}_{y} \\
& \hat{a}_{41}=\hat{U}\left\{\frac{\gamma-1}{2}\left(u^{2}+v^{2}\right)-H\right\} \quad \hat{a}_{42}=H \hat{n}_{x}-u \hat{U}(\gamma-1) \\
& \hat{a}_{43}=H \hat{n}_{y}-v \hat{U}(\gamma-1) \quad \hat{a}_{44}=\gamma \hat{U}
\end{aligned}
$$


where $\hat{U}=\left(n_{x} u+n_{y} y\right) J^{-1}|\nabla \xi|, \hat{n}_{x}=n_{x} J^{-1}|\nabla \xi|$, and $\hat{n}_{y}=n_{y} J^{-1}|\nabla \xi|$.

The elements of the Jacobian matrix, $\hat{m}_{j k}$, based on Roe's averaging are computed as follows

$$
\begin{aligned}
& \hat{m}_{11}=\left|\Lambda_{1}\right|+\frac{b q^{2}}{4} C_{1}-\frac{\bar{u}}{2 c} C_{3} \\
& \hat{m}_{12}=\frac{1}{2}\left(-b u C_{1}+\frac{C_{3}}{c} n_{x}\right) \\
& \hat{m}_{13}=\frac{1}{2}\left(-b v C_{1}+\frac{C_{3}}{c} n_{y}\right) \\
& \hat{m}_{14}=\frac{b}{2} C_{1}
\end{aligned}
$$$$
\hat{m}_{21}=\frac{b q^{2}}{4}\left(u C_{1}+n_{x} c C_{3}\right)-\frac{\bar{u}}{2 c}\left(u C_{3}+n_{x} c C_{2}\right)+\left|\Lambda_{1}\right| n_{x} \bar{u}
$$$$
\hat{m}_{22}=\left|\Lambda_{1}\right| n_{y}^{2}-\frac{b u}{2}\left(u C_{1}+n_{x} c C_{3}\right)+\frac{n_{x}}{2 c}\left(u C_{3}+n_{x} c C_{2}\right)
$$

$$
\hat{m}_{23}=-\left|\Lambda_{1}\right| n_{x} n_{y}-\frac{b v}{2}\left(u C_{1}+n_{x} c C_{3}\right)+\frac{n_{y}}{2 c}\left(u C_{3}+n_{x} c C_{2}\right)
$$

$$
\begin{aligned}
& \hat{m}_{24}=\frac{b}{2}\left(u C_{1}+n_{x} c C_{3}\right) \\
& \hat{m}_{31}=\left|\Lambda_{1}\right| n_{y} \bar{u}+\frac{b q^{2}}{4}\left(v C_{1}+n_{y} c C_{3}\right)-\frac{1}{2} \frac{\bar{u}}{c}\left(v C_{3}+n_{y} c C_{2}\right)
\end{aligned}
$$




$$
\begin{aligned}
& \hat{m}_{32}=-\left|\Lambda_{1}\right| n_{x} n_{y}-\frac{b u}{2}\left(v C_{1}+n_{y} c C_{3}\right)+\frac{n_{x}}{2 c}\left(v C_{3}+n_{y} c C_{2}\right) \\
& \hat{m}_{33}=\left|\Lambda_{1}\right| n_{x}^{2}-\frac{b v}{2}\left(v C_{1}+n_{y} c C_{3}\right)+\frac{n_{y}}{2 c}\left(v C_{3}+n_{y} c C_{2}\right) \\
& \hat{m}_{34}=\frac{b}{2}\left(v C_{1}+n_{y} c C_{3}\right) \\
& \hat{m}_{41}=\left|\Lambda_{1}\right|\left\{\bar{u}^{2}-\frac{q^{2}}{2}\left(1+\frac{b q^{2}}{2}\right)\right\}+\frac{b q^{2}}{4}\left(H C_{2}+\bar{u} c C_{3}\right)-\frac{\bar{u}}{2 c}\left(H C_{3}+\bar{u} c C_{2}\right) \\
& \hat{m}_{42}=\left|\Lambda_{1}\right|\left(\frac{q^{2}}{2} b u+u-n_{x} \bar{u}\right)-\frac{b u}{2}\left(H C_{2}+\bar{u} c C_{3}\right)+\frac{n_{x}}{2 c}\left(H C_{3}+\bar{u} c C_{2}\right) \\
& \hat{m}_{43}=\left|\Lambda_{1}\right|\left(\frac{q^{2}}{2} b v+v-n_{y} \bar{u}\right)-\frac{b v}{2}\left(H C_{2}+\bar{u} c C_{3}\right)+\frac{n_{y}}{2 c}\left(H C_{3}+\bar{u} c C_{2}\right) \\
& \hat{m}_{44}=\frac{b}{2}\left(H C_{2}-q^{2}\left|\Lambda_{1}\right|+c \bar{u} C_{3}\right)
\end{aligned}
$$

where nonstandard notation means $b=\frac{\gamma-1}{c^{2}}$ and

$$
\begin{aligned}
& C_{1}=\left|\Lambda_{3}\right|+\left|\Lambda_{4}\right|-2\left|\Lambda_{1}\right| \\
& C_{2}=\left|\Lambda_{3}\right|+\left|\Lambda_{4}\right| \\
& C_{3}=\left|\Lambda_{3}\right|-\left|\Lambda_{4}\right|
\end{aligned}
$$


The same as with FVS with higher order differencing, the left hand side is a block, $4 \times 4$ pentadiagonal matrix. To reduce the CPU time, the LU decomposition parts are vectorized over the lines. Although the memory requirement for the Jacobian matrix is substantial, our experience with an IBM 3090 with vector facility showed that the vectorized LU decomposition part is 3.5 times faster than scalar LU decomposition part ( based on a $85 \times 41$ block penta-diagonal matrix with 64 bit arithmatic).

To accelerate the convergence rate, a local time step (or variable time step , space varying time step ) is used. A local time step has been widely used to accelerate the convergence rate of viscous flow computations (Refs. 15,72 ). Following Jameson (Ref. 73), to use a local time step is equivalent to solving ( for Euler Equations )

$$
\frac{\partial Q}{\partial t}+\alpha\left[\frac{\partial F}{\partial x}+\frac{\partial G}{\partial y}\right]=0
$$

where $\alpha$ is a variable scale factor. McDonald and Briley (Ref. 74) interpreted the local time step as a simple matrix conditioning by a diagonal matrix and explained the faster convergence of using a local time step. Turkel (Ref. 75) also interpreted the local time step as introducing an artificial wave speed that increases as one goes to the farfield.

The equation for the local time step used in this case is (Ref. 76) 


$$
\Delta t=\frac{C F L}{\left|u_{\xi}\right|+\left|u_{\eta}\right|+c(|\nabla \xi|+|\nabla \eta|)}
$$

which means a more uniform CFL number over the computational grid. Although the steady state solution is not affected, the local time step destroys the time accuracy of the numerical method, and a transient solution does not have a physical meaning. 


\subsubsection{Jacobians of Viscous Terms}

The linearization of the viscous terms is based on the central difference as given in Section 3.5 and can be expressed as the following. From eq. (39) and (47)

$$
\begin{aligned}
\Delta \hat{G}_{v} & =\frac{\partial}{\partial \eta}\left(\hat{H}_{2} \Delta Q\right) \\
& =\frac{\partial}{\partial \eta}\left[J\left(\frac{|\nabla \xi|}{J}\right)^{2} \mu \frac{\partial \hat{T}}{\partial Q} \Delta Q\right]
\end{aligned}
$$

Defining $\hat{H}=\frac{\partial \hat{T}}{\partial Q}$, then

$$
\begin{aligned}
{\left[\frac{\partial \Delta \hat{G}_{v}}{\partial \eta}\right]_{k} } & =\frac{\partial^{2}}{\partial \eta^{2}}\left[J\left(\frac{|\nabla \xi|}{J}\right)^{2} \mu \hat{H} \Delta Q\right] \\
& =\frac{\partial}{\partial \eta}\left[J\left(\frac{|\nabla \xi|}{J}\right)^{2} \mu \hat{H} \Delta Q\right]_{k+1 / 2}-\frac{\partial}{\partial \eta}\left[J\left(\frac{|\nabla \xi|}{J}\right)^{2} \mu \hat{H} \Delta Q\right]_{k-1 / 2}
\end{aligned}
$$

Let $\Delta \eta=1$, and eq. (54) becomes

$$
\begin{aligned}
\frac{\partial}{\partial \eta}\left[J\left(\frac{|\nabla \xi|}{J}\right)^{2} \mu \hat{H} \Delta Q\right]_{k+1 / 2} & =\left[J\left(\frac{|\nabla \xi|}{J}\right)^{2}\right]_{k+1 / 2} \frac{\partial}{\partial \eta}[\mu \hat{H} \Delta Q]_{k+1 / 2} \\
& =\left[J\left(\frac{|\nabla \xi|}{J}\right)^{2}\right]_{k+1 / 2}\left[[\mu \hat{H} \Delta Q]_{k+1}-[\mu \hat{H} \Delta Q]_{k}\right]
\end{aligned}
$$

The elements of the viscous Jacobian matrix, $\hat{h}_{j k}$, are given by

$$
\hat{h}_{11}=0 \quad \hat{h}_{12}=0 \quad \hat{h}_{13}=0 \quad \hat{h}_{14}=0
$$




$$
\begin{aligned}
& \hat{h}_{21}=-\frac{1}{\rho}\left\{\left(1+\frac{1}{3} t_{x}^{2}\right) u+\frac{1}{3} t_{x} t_{y} v\right\} \quad \hat{h}_{22}=\frac{1}{\rho}\left(1+\frac{1}{3} t_{x}^{2}\right) \\
& \hat{h}_{23}=\frac{1}{3 \rho} t_{x} t_{y} \quad \hat{h}_{24}=0 \\
& \hat{h}_{31}=-\frac{1}{\rho}\left\{\left(1+\frac{1}{3} t_{y}^{2}\right) v+\frac{1}{3} t_{x} t_{y} u\right\} \quad \hat{h}_{32}=\frac{1}{3 \rho} t_{x} t_{y} \\
& \hat{h}_{33}=\frac{1}{\rho}\left(1+\frac{1}{3} t_{y}^{2}\right) \quad \\
& \hat{h}_{41}=-\frac{1}{\rho}\left\{\left(1+\frac{1}{3} t_{x}^{2}\right) u^{2}+\left(1+\frac{1}{3} t_{y}^{2}\right) v^{2}+\frac{2}{3} t_{x} t_{y} u v-\frac{\gamma}{P r}\left(q^{2}-\frac{e}{\rho}\right)\right\} \\
& \hat{h}_{42}=\frac{1}{\rho}\left\{\left(1+\frac{1}{3} t_{x}^{2}\right) u+\frac{1}{3} t_{x} t_{y} v-\frac{\gamma}{\operatorname{Pr}} u\right\} \\
& \hat{h}_{43}=\frac{1}{\rho}\left\{\left(1+\frac{1}{3} t_{y}^{2}\right) v+\frac{1}{3} t_{x} t_{y} u-\frac{\gamma}{\operatorname{Pr}} v\right\} \\
& \hat{h}_{44}=\frac{1}{\rho} \frac{\gamma}{\operatorname{Pr}}
\end{aligned}
$$

52 


\subsection{Results and Discussions}

In order to test the numerical procedure, several test cases were chosen. The first test problem is the compressible laminar boundary layer on a flat plate. For this case, an exact similarity solution exists. The purpose of this test is to compare the numerical solution with an exact solution for a viscous simple case. The second case is the laminar boundary layer - shock interaction problem, and this problem was studied experimentally by Hakkinen et al (Ref. 93). This case represents a step forward in flow complexity. It also involves waves in the viscous region. The final and most complex case studied is the supersonic slot injection problem studied experimentally by Gilreath (Ref. 1) and others. 


\subsection{Compressible Laminar Boundary Layer over a Flat}

\section{Plate}

The first test case chosen is the supersonic laminar boundary layer over a flat plate. The flow conditions studied here is a free stream Mach number of 1.98 with a Reynolds number per foot is $1.92 \times 10^{5}$. This condition corresponds to the conditions of the supersonic slot injection studies of Gilreath. The physical domain of this problem is shown in Fig. 3.

For the case of a subsonic laminar boundary layer $(M=0.5)$, several reseachers performed extensive numerical calculations for verifying numerical solutions. Thomas and Walters (Ref. 35) compared their numerical results based on van Leer's flux vector splitting with the Blasius solution. Schroeder and Haenel (Ref. 77) also performed the same numerical experiments. Rudy and Strikwerda (Ref. 78) studied the effect of numerical boundary conditions by solving the subsonic laminar boundary layer over a flat plate using the MacCormack scheme. The reason for choosing the supersonic laminar boundary layer for study here instead of choosing the subsonic laminar boundary layer is to compare both the temperature and velocity field based on the numerical method with the similarity solution (Ref. 79) without any complicating effect of a leading edge. Since the upwind method based on the FDS and FVS has been criticized for the fact that the predicted total enthalpy is not constant in the inviscid steady state (Refs. 73 , 80), it seems worthwhile to consider the temperature field in detail. Also, this 
step is a preliminary work for the more complex problem such as the supersonic slot injection problem.

At this point, it is proper to mention the problem of numerical boundary conditions. As pointed by Moretti two decades ago (Ref. 81), the problem of numerical boundary conditions is a very important but difficult problem (Refs. 82 - 84). For the case of hyperbolic partial differential equations, there exists a well developed theory based on mathematics. The unsteady, Euler equation is hyperbolic in time, and there have been great achievements in the development of numerical boundary schemes (Ref. 85) . But, for the unsteady compressible Navier-Stokes equations, one does not know enough about the equations mathematically, and even the classification of partial differential equation is not possible (Ref. 86) . Some mathematicians have called these equations incompletley parabolic (Refs. $87-88$ ). The numerical boundary conditions for the Navier-Stokes equations developed to date are largely based on physical reasoning and considering the unsteady, Navier-Stokes equations as a mixed set of hyperbolic - parabolic equations in time (Ref. 47) .

The numerical boundary conditions used in this case are as follows. At the inflow boundary, if $M>1$ then all the variables based on the similarity solution are specified. If $M<1, u, v$ and $T$ are specified and pressure is calculated by a linearized characteristic relation as 


$$
\begin{aligned}
& u_{1}^{n+1}=u_{1}^{n} \\
& v_{1}^{n+1}=v_{1}^{n} \\
& T_{1}^{n+1}=T_{1}^{n} \\
& p_{1}^{n+1}=p_{2}^{n+1}-\rho_{1}^{n} c_{1}^{n}\left(u_{2}^{n+1}-u_{1}^{n+1}\right)
\end{aligned}
$$

This boundary condition was used by Rudy and Strikwerda and showed good results for subsonic laminar boundary layer flow. This boundary condition was also studied by Cline (Ref. 92), who included the viscous terms in the characteristic relation as a source term. At the outflow boundary, all the variables are calculated from the interior points by 1 st order extrapolation. At the wall, velocities and temperature are determined from the no-slip and adiabatic condition. The wall pressure is determined from the relation of $\frac{\partial p}{\partial y}=0$. At the upper boundary, variables are determined from simple wave extrapolation (Refs. $89-90$ ). Convergence to steady state was assumed, when the L-2 norm of the residual is reduced to $10^{-4}$ of its initial value. The computational grid used for this case is a uniform $\mathrm{x}$-grid, and the y-direction is stretched from the following relation (Refs. 47,91$)$.

$$
y=\frac{(\beta+1)-(\beta-1)\left[\frac{\beta+1}{\beta-1}\right]^{1-\eta}}{\left[\frac{\beta+1}{\beta-1}\right]^{1-\eta}+1}
$$

where $\beta$ is a stretching parameter taken in this study $\beta=1.03$. With this value of $\beta$, approximately 36 percent of the grid points are located in the boundary 
layer, and minimum spacing in the y-direction to initial thermal boundary layer thickness is approximately $1 / 15,1 / 30$, and $1 / 45$ for $k_{\max }=21,31$, and 41 respectively. A typical $41 \times 41$ grid is shown in Fig. 4 .

The results from Roe's method $\left(\kappa_{x}=-1, \kappa_{y}=1 / 3\right)$ with a $41 \times 41$ grid showed very good comparisons with the exact similar solution. As shown in Figs. 5-6, the velocity and temperature at 4 different streamwise locations showed excellent similar characteristics. The effects of grid spacing on the accuracy of the numerical solution are shown in Figs. 7 and 8. Velocity and temperature with a very coarse $21 \times 21$ grid showed almost grid converged results.

The computational time with Roe's method is $2.6 \times 10^{-4}$ sec per grid point per time step on an IBM 3090 with vector facility, and a typical convergence history is shown in Fig. 9.

On the other hand, the results from van Leer's FVS method are not as good as Roe's FDS method. When compared with the exact similar solution (Figs. 10-11), the agreements are not satisfactory even with a $41 \times 41$ grid. As shown in Figs. 12 and 13, the effects of grid spacing on the accuracy of FVS are more severe than FDS.

Recently, van Leer et al (Ref. 45) obtained similar results for a one-dimensional conical Navier-Stokes flow. They concluded that for one-dimensional conical flow the results obtained with Roe's FDS are far more accurate than those of van 
Leer's FVS. They explained the reasons as smaller numerical dissipation of Roe's FDS than van Leer's FVS. Our results with an attached 2-D compressible boundary layer flow confirm their conclusions. It was not judged worthwhile to pursue grid adaptation for this simple problem. 


\subsection{Laminar Boundary Layer - Shock Interaction}

The second sample case is the laminar boundary layer - shock interaction problem. A sketch of this problem is, which was taken from Ref. 11., is shown in Fig. 14. This problem corresponds to the experiments of Hakkinen et al (Ref. 93) at a free stream Mach number of 2 and Reynolds number of $0.296 \times 10^{6}$ based on the shock location. For this case, the shock is strong enough to separate the flow field, and the shock wave angle is 32.585 degree.

Several researchers have studied this problem by various numerical methods. MacCormack (Refs. 11, 94), Li (Ref. 95) and Shang et al (Ref. 96) used the explict MacCormack method, and Beam and Warming (Ref. 14) compared their method with experimental data. Recently, Thomas and Walters (Ref. 35) performed extensive numerical experiments using the FVS of van Leer. The computational grid used for this case is equivalent to that of Beam and Warming except that the computational domain is extended vertically to eliminate shock reflection from the upper boundary as was done by Thomas and Walters. A typical $32 \times 57$ grid is shown in Fig. 15. Numerical boundary conditions for this problem are as follows. At the supersonic inflow boundary, free stream conditions were imposed for cells below the shock location and for cells above the shock, post-shock conditions were specified. Along the upper boundary, post-shock conditions were specified. No slip and adiabatic wall conditions were imposed on the plate with symmetry conditions ahead of the plate. At the 
outflow boundary, all variables were obtained by extrapolation from the interior points.

Fig. 16 presents the wall pressure distribution based on Roe's FDS method compared to data. As shown in this figure, the surface pressure distribution from Roe's method showed good agreement compared with Hakkinen's experimental data. Experimental data were read from the graph of Hakkinen's work, and it must be pointed out that due to scaling, some reading errors were inevitable. The numerical pressure distribution shows that the pressure plateau, which is typical of this kind of separated flow, is captured well with a rather coarse $32 \times 57$ grid. In Fig. 17, the skin friction distribution of the present calculation and experimental data are presented. The predicted length of the separated zone is greater than that of the experiment, but it shows a near " dual minimum " ( or W-shape ) (Refs. 95 - 96) distribution. Important flow patterns such as the leading edge shock, the expansion near the separated zone and the reflected shock are well captured.

Wall pressure and skin friction distribution calculated from van Leer's FVS method are shown in Figs. 18-19. With a $32 \times 57$ grid, a pressure plateau is not predicted in numerical results. The estimated separated zone from this method is somewhat smaller than that of Roe's FDS method.

To study the effect of grid refinement, grid spacing in the x-direction was halved (63 x 57 grid). Roe's method with this fine grid showed improvements when 
compared with experimental data. Pressure oscillation after the shock is reduced, and the pressure plateau is clearer as shown in Fig. 20. As shown in this figure, predicted wall pressure distribution from both $32 \times 57$ and $63 \times 57$ grid showed good agreement with the experimental data. Fig. 21 shows the comparison of the skin friction coefficient distribution. Pressure contour plotting is depicted in Fig. 22. When compared with the experimental data, the length of the separated zone from a $63 \times 57$ grid is reduced, and the skin friction prediction in the reattached zone is improved. Due to memory limits of the IBM 3090, a further grid refinement study could not be performed. Comparisons of the wall skin friction coefficient distribution from the present Roe's FDS method with experiment and other computations are shown in Fig. 23. The computations with the present method and Thomas and Walters (Ref. 35) were with thin layer approximation, while the results of MacCormack and Baldwin (Ref. 11, 94) were with the complete Navier-Stokes equations. As shown in this figure, the predicted length of the separated zone from the present Roe's FDS method is slightly longer than those of others. Since our grid refinement study showed the correct trends of reduced separated zone as the grid is refined, it is anticipated that if finer grid were used, the agreement with the experimental data would be improved. Despite the differences in the levels of skin friction in the separated and reattached zone, there is generally good agreement between all of the numerical results and experiment.

Although Walters and Thomas had performed extensive numerical computations using van Leer's FVS, the fine grid results. of the present van Leer's FVS is 
presented for completeness. The pressure contour from a $63 \times 57$ grid is shown in Fig. 24. Although grid refinement improved the wall pressure distribution, the agreement is not impressive (Fig. 25). The skin friction distribution from FVS compared well with the experimental data (Fig. 26), but following Thomas and Walter's work, this does not seem to be a grid convergent result. Their very fine grid $(91 \times 169)$ results showed similar result when compared with the present calculations using Roe's method.

When compared with experimental data and indications from other numerical works, Roe's FDS method is judged superior to van Leer's FVS for the laminar boundary layer - shock interaction problem. Because Roe's scheme has numerical dissipation which is proportional to the absolute value of the eigenvalue and if one of these eigenvalues is small then numerical dissipation corresponding to that eigenvalue is also small. This might be one reason for the better performance of Roe's FDS method observed here. Again, this problem was not judged complex enough to merit grid adaptation. 


\subsection{Supersonic Slot Injection Problem}

The third test case considered is a supersonic slot injection problem. This is a truly complex flow involving interactions of waves and shear layers. For this problem, experimental data from experiments done by Gilreath (Ref. 1) in an atmospheric intake wind tunnel were available. Experiments were air to air injection, the slot height was 0.5 inch and the free stream Reynolds number per foot was 1.3 million. Free stream and jet Mach numbers were 4.19 and 1.98, respectively. The static pressure at the slot exit was slightly greater than that of the undisturbed freestream (underexpanded jet ).

Calculated wall pressure distribution and numerically generated flow fields are compared with the experimental data. The numerical boundary conditions used in this problem are same as for the compressible laminar boundary layer problem. Since Roe's FDS method performed better than van Leer's FVS method, only the results from Roe's FDS method will be presented for the supersonic slot injection problem. For this problem, adaptive grid methods are implemented in the numerical procedure, and the advantages and limitations of combined numerical methods are discussed in detail. 


\subsubsection{Injection without Wedge}

This case corresponds to the arrangement in Fig. 1a. To generate the numerical boundary conditions at the inflow boundary, the boundary layer thickness at the slot exit was measured from the experimental Schlieren picture. Since light intensity in a Schlieren picture is proportional to the density gradient, the measured thermal boundary layer thickness from the Schlieren picture might be slightly different from the usual definition of thermal boundary layer thickness. The experiment was done in an indraft tunnel, and with this flow condition, Gilreath (Ref. 1) presumed that density changes less than 6 percent can not be discerned from the photograph. Experimental pressures were measured by an inclined tube manometer, and errors in measuring the pressure are believed to be \pm 2 percent. With known experimental conditions, the velocity and temperature distribution were computed from the similarity solutions. The reported experimental condition for the jet Mach number at the slot exit was changed slightly from 1.98 to 1.95 , since a $M_{j}=1.98$ input gave too low a pressure at the slot exit compared to the experimental data at the neighboring point.

The first computational grid used in this case was uniform $\mathrm{x}$-grid and $\mathrm{y}$ - direction stretched by eq. (56). The outflow boundary is taken at $\mathrm{X} / \mathrm{H}=8$ and the upper boundary is located at $\mathrm{Y} / \mathrm{H}=3.6$, where $\mathrm{H}$ is the slot height. A typical $51 \times 70$ grid is shown in Fig. 27. With a $51 \times 70$ grid, 40 grid points are located between the splitter plate and wall. Approximately 13 points are located in the boundary 
layer at the slot exit and, at this point, the minimum y - grid spacing to boundary layer thickness is 2.5 percent.

The predicted wall pressure distribution from Roe's FDS method is compared with experimental data in Fig. 28. Although generally good agreement was achieved, the calculated pressure after the shock impingement is lower than the experimental values. Considering the complexity of the supersonic slot injection problem, the agreement is satisfactory. The skin friction distribution at the wall is shown in Fig. 29. Since experimental skin friction data are not available, it is not possible to compare with the theoretical values. For this case, the flow does not separate, and the Schlieren picture confirms this results. Contour plotting of both pressure and density are presented in Figs. 30-31. For this plotting, a total of 21 contour lines were plotted. Although somewhat smeared, important wave patterns are described well.

Before studying the effect of grid refinement, we first studied the effects of using single precision (32 bit). As shown in Figs. 32-34, single precision does not effect the accuracy of the results nor the convergence rate for this problem.

To study the effects of grid refinement, a $81 \times 90$ grid (Fig.35) with single precision was used. In Figs. 36-37, the wall pressure and skin friction distributions with a fine grid $(81 \times 90)$ are compared with the results of the coarse grid. Pressure and density contour plots from the $81 \times 90$ grid are shown 
in Figs. 38-39. As shown in these figures, both the expansion and the shock are captured more clearly, but the difference is small.

One interesting feature of the slot injection problem is the formation of a lip shock followed by an expansion wave. Hama (Ref. 97) studied the lip shock which emanated from the separated edge of a 6 degree half-angle wedge experimentally. His conclusion was that although he does not completely preclude the effect of inviscid rotationality, the viscous separation effect is directly responsible to the lip shock formation. To study the effect of inviscid rotationality, the Euler equations were solved here with the $81 \times 90$ grid. As can be seen in Fig. 40, both the strength of the lip shock and the expansion fan are weaker than that of the viscous case. These results confirm the conclusion of Hama that viscosity played a primary role in the formation of the lip shock. The effect of inviscid rotationality on the formation of the lip shock is small but not negligible. Contour plots of pressure and density for the inviscid calculation are shown in Figs. 41 and 42 . Figure 43 shows that quadratic convergence was obtained, since the flowfield is supersonic.

The results from the $81 \times 90$ adaptive grid are shown in the next figures. First, the resulting adaptive grid is shown in Fig. 44. This grid was generated from the equidistribution law as explained in Chap. 2. This grid clearly shows the wave system produced by slot injection. Both the pressure and density contour and wall pressure distribution from the adaptive grid showed improvements over the non-adaptive grid results (Figs. 45-47). When compared with experimental 
Schlieren picture, it showed the correct positions and angles of the shocks and expansion waves. The skin friction coefficient from the adapative grid is compared to that of the non-adaptive grid in Fig. 48. Since expansion waves are more clearly captured by the adaptive grid, the maximum skin friction coefficient from the adaptive grid is higher than that of the non-adaptive grid. Despite the simplicity of the adaptive grid method based on the equidistribution law, it needs extensive numerical experiments to generate smooth grids for complex flow problems such as supersonic slot injection problems.

Flow fields generated from the numerical solutions are compared with experimental pictures. Figure 49 shows the numerical interferogram and experimental pictures. The Schlieren picture is compared with isolines of $\frac{\partial \rho}{\partial y}$ in Fig. 50. The agreement between the experimental and theoretical photos is generally very good.

\subsubsection{Injection over a 10-Degree Wedge}

The next problem treated is a supersonic slot injection over a 10 degree wedge surface as sketched in Fig. 1b. For this problem, the experimental Schlieren picture showed a separated region and very complex viscous and inviscid interaction. 
The computational domain is extended to $12 \mathrm{H}$ in the $\mathrm{x}$-direction and $6 \mathrm{H}$ in the y-direction. The $65 \times 70$ grid used for this case is shown in Fig. 51. First, pressure and density contours are shown in Figs. 52 and 53, where the dividing stream line is plotted as a broken line. The wall pressure prediction from the 65 $x 70$ grid is compared with the experimental data in Fig. 54. Predicted wall pressure is higher than the experimental data. Since skin friction was not measured experimentally, it was not possible to directly compare the calculated skin friction coefficient (Fig. 55). The predicted results showed that near the outflow boundary, the flow is attached at only two axial stations, so the adequacy of the numerical boundary condition at the outflow boundary can be questioned.

To study the effects of the outflow boundary location, the computational domain was further extended to $15 \mathrm{H}$ in the $\mathrm{x}$-direction and $7.5 \mathrm{H}$ in the y-direction with same $\Delta x$. The effects of the outflow boundary location on the skin friction and the wall pressure are shown in Figs. 56 and 57. As shown in Fig. 56, the reattachment point moved downstream as $x_{\max }$ increased. This result showed the importance of the numerical boundary conditions at the outflow boundary. The predicted wall pressure with $x_{\max }=15 \mathrm{H}$ case is higher than that of $x_{\max }=12 \mathrm{H}$ (Fig. 57). The experimental Schlieren picture is compared with the isolines of constant $\frac{\partial \rho}{\partial y}$ in Fig. 58. As we can see in this figure, the bouncing waves between the shear layer and the separated boundary layer are not clearly captured, and the predicted separated boundary layer showed a somewhat distorted shape. 
The major reasons for the discrepancies between the numerical and experimental data is not clear. Since the experimental Schlieren picture showed some fluctuations in the shear layer at $\mathrm{X}=10 \mathrm{H}$, it is conjectured that the shear layer was transitional or turbulent afterwards. Although it is not clear from the experimental Schlieren picture, there is a possibility that the reattached boundary layer was turbulent in the experimental condition. Since the present numerical method does not have the capability to analyze the transition and turbulence phenomena, it is believed that laminar flow assumption for this case might not be adequate.

Also, it is possible that $3 \mathrm{D}$ effects were important in the large separation region of the experiments. Few, large separation zones are truly 2D. Of course, the present calculation, which is limited to $2 D$, could not capture any such effects. Further grid refinement study and the implementation of an adaptive grid method was not pursued for this case because of the above-mentioned reasons. 


\subsection{Concluding Remarks}

To understand supersonic slot injection problems numerically, a computational procedure based on the upwind method with an adaptive grid was developed.

To test the accuracy of the numerical methods, the compressible laminar boundary layer over a flat plate and the laminar boundary layer - shock interaction problems were chosen as sample cases. For these problems, Roe's FDS method and van Leer's FVS method were compared with exact solutions and experimental data. Comparisons showed that Roe's FDS method is more accurate than van Leer's FVS method for these viscous problems. The reasons for the better performance of Roe's FDS can be explained by the smaller numerical dissipation than from van Leer's FVS.

For the supersonic slot injection problem without wedge case, Roe's FDS method with an adaptive grid method showed very promising results. One advantage of an adaptive grid method based on the equidistribution law is its simplicity. One 
of the drawbacks of this adaptive grid method is that it needs extensive numerical experiments to generate a smooth grid for complex flow problems.

For the slot injection over a 10 degree wedge surface, numerically predicted wall pressure distribution showed poorer agreement with the experimental data. To eliminate the problems of numerical outflow boundary conditions, the computational domain was extended to $X=15 \mathrm{H}$. The experimental Schlieren picture showed slight fluctuations of the shear layer from the splitter plate near $\mathrm{X}=10 \mathrm{H}$, and it is believed that the shear layer was turbulent afterwards. This transition to turbulence phenomena in the computational domain invalidated the laminar flow assumption in the calculations. The major reason for the discrepancy of numerical and experimental data might be explained by this transition to turbulent phenomena and / or 3D effects in the separated zone in the experiments. 


\section{References}

1. Gilreath, H. E., "An Investigation of Gaseous Tangential Injection in Supersonic Flow," Ph. D. Thesis, University of Maryland, Apr. 1968.

2. Schetz, J. A., and Gilreath, H. E., "Tangential Slot Injection in Supersonic Flow," AIAA Journal, Vol. 5, Dec. 1967, pp. 2149-2154.

3. Goldstein, R. J., Eckert, E. R. G., Tsou, F.K., and Haje-Sheikh, A., "Film Cooling with air and Helium Injection through a RearwardFacing Slot into a Supersoni Air Flows," AIAA Journal, Vol. 4, Jun. 1966, pp. 981-985.

4. Kenworthy, M., and Schetz, J. A., "Experimental Study of Slot Injection into a Supersonic Stream," AIAA Journal, Vol. 11, May, 1973, pp. 585-586.

5. Walker, D. A., Campbell, R. L., and Schetz, J. A., "Turbulence Measurements for Slot Injection in Supersonic Flow," AIAA Paper 88-0123, Jan. 1988.

6. Libby, P. A., and Schetz, J. A., "Approximate Analysis of the Slot Injection of a Gas in Laminar Flow," AIAA Journal, Vol. 1, May, 1963, pp. 1056-1061.

7. Schetz, J. A., and Jannone, J., "Initial Boundary Layer Effects on Laminar Flows with Wall Slot Injection," Journal of Heat Transfer, Vol. 87, Feb. 1965, pp. 157-160.

8. Chapman, D. R., "Computational Aerodynamics Development and Outlook," AIAA Journal, Vol. 17, Dec. 1979, pp. 1293-1313. 
9. Schroenauer, W. and Gentzsch, W., "The Efficient Use of Vector Computers with Emphasis on Computational Fluid Dynamics," Notes on Numerical Fluid Mechanics, Vol. 12, 1986.

10. Shang, J. S., Buning, P. G., Hankey, W. L. , and Wirth, M. C., "Performance of a Vectorized Three-Dimensional Navier-Stokes Code on the CRAY-1 Computer," AIAA Journal, Vol. 18, Sep. 1980, pp. 1073-1079.

11. MacCormack, R. W. and Baldwin, B. S., "A Numerical Method for Solving the Navier-Stokes Equations with Application to Shock-Boundary Layer Interactions," AIAA Paper 75-1, Jan. 1975.

12. Shang, J. S. and Hankey, W. L. , "Numerical Solution of the Navier-Stokes Equations for a Three-Dimensional Corner," AIAA Paper $77-169$, Jan. 1976.

13. Scott, J. N. and Hankey, W. L. , "Numerical Simulation of Cold Flow in an Axisymmetric Centerbody Combustor," AIAA Journal, Vol. 23, May, 1985, pp. 641-649.

14. Drummond, J. P., "Numerical Study of a Ramjet Dump Combustor Flowfield," AIAA Journal, Vol. 23, Apr. 1985, pp. 604-611.

15. Jameson, A., Schmidt, W., and Turkel, E., "Numerical Solutions of the Euler Equations by Finite Volume Methods Using Runge-Kutta Time Stepping Schemes," AIAA Paper 81-1259, Jun. 1981.

16. Beam, R. M. and Warming, R. F. , "An Implicit Factored Scheme for the Compressible Navier-Stokes Equations," AIAA Journal, Vol. 16, Apr. 1978, pp. 393-402.

17. Pulliam, T. H. and Steger, J. L., "Recents Improvements in Efficiency, Accuracy, and Convergence for Implicit Approximate Factorization Algorithms," AIAA Paper 85-0360, Jan. 1985.

18. Pulliam, T. H. , "Implicit Solution Methods in Computational Fluid Dynamics," Applied Numerical Mathematics 2, 1986, pp. 441-474.

19. Chakravarthy, S. R., "Development of Upwind Schems for the Euler equations," NASA CR-4043, 1987.

20. Steger, J. L., and Warming, R. F., "Flux Vector Splitting of the Inviscid Gasdynamic equations with Application to Finite Difference Methods," Journal of Computational Physics, Vol. 40, 1981, pp. 263-293. 
21. van Leer, B., "Flux Vector Splitting for the Euler Equations," Lecture Notes in Physics, Vol. 170, 1982, pp. 507-512.

22. Roe, P. L., "Approximate Riemann Solvers, Parameter Vectors," and Difference Scheme," Journal of Computational Physics, Vol. 43, 1981, pp. 357-372.

23. Harten, A., Lax, P. D., and van Leer, B., "On Upstream Differencing and Godunov-Type Schemes for Hyperolic Conservation Laws," SIAM Review, Vol. 25, No. 1, Jan. 1983, pp. 35-61.

24. van Leer, B., "On the Relation Between the Upwind-Differencing Schemes of Godunov, Engquist-Osher, and Roe," SIAM J. Sci. Stat. Comput., Vol. 5, No. 1, Mar. 1984, pp. 1-20.

25. Harten, A., "On a Class of High Resolution Total-Variation-Stable Finite Schemes ," SIAM Journal of Numer. Anal., Vol.21, Feb. 1984. , pp. 1-23.

26. Harten, A., "High Resolution Schemes for Hyperbolic Conservation Laws," Journal of Computational Physics, Vol. 49,.1983, pp. 357-393.

27. Walters, R. W. and Thomas J. L., "Advances in Upwind Relaxation Methods," State of the Art Surveys on Computational Mechanics, Special ASME publication.

28. Roe, P. L., "Characteristic-Based Schemes for the Euler Equations," Ann. Rev. Fluid Mech., Vol. 18, 1986, pp. 337-365.

29. Mulder, W. A., and van Leer, B., "Implicit Upwind Methods for the Euler Equations," AIAA Paper 83-1930, 1983.

30. Yee, H. C., Warming, R. F., and Harten, A., "Implicit Total Variation Diminishing ( TVD ) Schemes for Steady- State Calculations," Journal of Computational Physics, Vol. 57, pp. 327-360.

31. Anderson, W.K., Thomas, J.L., and van Leer, B., "A Comparison of Finite Volume Flux Vector Splittings for the Euler Equations," AIAA Paper 85-0122, Jan. 1985.

32. Walters, R. W., and Dwoyer, D. L., "An Efficient Iterarion Strategy for the Solution of the Euler Equations," AIAA Paper 85-1529, Jul. 1985.

33. Grossman, B., and Walters, R. W., "An Analysis of Flux-Split Algorithm for Euler's Equations with Real Gas," AIAA Paper 87-1117, Jun. 1987. 
34. Chakravarthy, S. R., Szema, K. Y., Goldberg, U. C., and and Gorski, J. $\mathrm{J}$. "Applicatin of a New Class of High Accuarcy TVD Schemes to the Navier-Stokes Equations," AIAA Paper 85-0165, Jan. 1985.

35. Thomas, J. L., and Walters, R. W., "Upwind Relaxation Algorithms for the Navier-Stokes Equations," AIAA Paper 85-1501, Jul. 1985.

36. Candler, G. V., and MacCormack, R. W., "Hypersonic Floow Past 3-D Configurations," AIAA Paper 87-0480, Jan. 1987.

37. Lawrence, S. L.,Tannehill, J. C., and Chaussee, D. S., "An Upwind Algorithm for the Parabolized Navier-Stokes Equations," AIAA Paper 86-1117, May 1986.

38. Lawrence, S. L., Chaussee, D. S., and Tannehill, J. C., "Application of an Upwind Algorithm to the Three Dimensional Parabolized Navier-Stokes Equations," AIAA Paper 87-1112, Jun. 1987.

39. Newsome, R. W., Walters, R. W., and Thomas, J. L., "An Efficient Iteration Strategy for Upwind/Relaxation Solutions to the Thin-Layer Navier-Stokes Equations," AIAA Paper 87-1113, Jun. 1987.

40. Thompson, J. F., "Grid Generation in Computational Fluid Dynamics," AIAA Journal, Vol. 22, Nov., 1984, pp. 1505-1523.

41. Thompson, J. F., Warsi, Z. U. A., and Mastin C. W., "Boundary-Fitted Coordinate Systems for Numerical Solutions of Partial Differential Equations - A Review," Journal of comutational Physics, Vol. 47, 1982, pp. 1-108.

42. Thompson, J. F., Warsi, Z. U. A., and Mastin, C. W., "Numerical Grid Generation, Foundation, and Applications," North-Holland, 1985.

43. Anderson, D. A. , and Steinbrenner, J., "Generating Adaptive Grids with a Conventional Grid Scheme," AIAA Paper 86-0427, Jan. 1986.

44. Anderson, D. A. , “Adaptive Grid Scheme Controlling Cell Area/Volume , AIAA Paper 87-0202, Jan. 1987.

45. van Leer, B. et al, "A comparison of Numerical Flux Formula for the Euler and Navier-Stokes Equations," AIAA Paper 87-1104, Jun. 1987.

46. Eiseman, P. R. , "Grid Generation for Fluid Mechanics Computations," Annual Review of Fluid Mechanics,, Vol. 17, 1985, pp. 487-522. 
47. Anderson, D. A., Tannehill, J. C., and Pletcher, R. H., Computational Fluid Mechanics and heat transfer, McGraw Hill Book Company, 1984.

48. MacCormack, R. W., and Paullay, A. J., "The Influence of Computational Mesh on a Accuracy for Initial Value Problems with Discontinuous or Nonunique Solutions," Computers \& Fluids, Vol. 2, 1974, pp. 339-361.

49. Davis, S. F., "A Rotationally biased Upwind Difference Scheme for the Euler Equations," Journal of Computational Physics, Vol. 63, 1986, pp.65-92.

50. Hsu, C. C., and Tu, C. G., "A Self-Adaptive Gridding for Inviscid Transonic Projectile Aerodynamics Computations," International Journal for Numerical Methods in Fluids, Vol. 7, 1987, pp. 567-579.

51. Brackbill, J. U., and Saltzman, J. S., "Adaptive Zoning for Singular Prolems in Two Dimensions," Journal of Computational Physics, Vol. 46, 1982, pp. 343-368.

52. Dannenhoffer, J. F., and Baron, J. R., "Grid Adaption for the 2-D Euler Equations," AIAA Paper 85-0484, Jan. 1985.

53. Dwyer, H. A., Kee, R. J., and Sanders, B. R., "Adaptive Grid Method fo Problems in Fluid Mechanics and Heat Transfer," AIAA Journal, Vol. 18, Oct. 1980. pp. 1205-1212.

54. Dwyer, H. A. , "Grid Adaption for Problems with Separation, Cell Reynolds Number, Shock-Boundary Layer Interaction, and Accuracy," AIAA Paper 83-0449, Jan. 1983.

55. Gnoffo, P. A., "A Vectorized, Finite-Volmue, Adaptive - Grid Algorithm for Navier-Stokes Calculations," Numerical Grid Generation, edited by Thompson, J. F., 1982, pp. 819-835.

56. Nakahashi, K., and Deiwert, G. S., "A Three-Dimensional Adaptive Grid Method," AIAA Paper 85-0486, 1985.

57. Nakahashi, K., and Deiwert, G. S., "A Self-Adaptive Grid method with Application to Airfoil Flow," AIAA Paper 85-1525, Jul. 1985.

58. Abolhassani, J. S., Smith, R. E., and Tiwari, S. N., "Grid Adaption for Hypersonic Flow," AIAA Paper 87-1169, 1987.

59. Venkatapathy, E., Lombard, C. K., Bardina, J., and Luh, R. C. C., "Accurate Numerical Simulation of Supersonic Jet Exhaust Flow with 
CSCM on Adaptive Overlapping Grids," AIAA Paper 87-0465, Jan. 1987.

60. Berger, M. J., and Jameson, A., "Automatic Adaptive Grid Refinement for the Euler equations," AIAA Journal, Vol. 23, Apr. 1985, pp.561-568.

61. Berger, M. J., and Oliger, J., "Adaptive Mesh Refinement for Hyperbolic Partial Differential Equations," Journal of Computational Physics, Vol. 53, 1984, pp. 484-512.

62. Ni, R. H., "A Multiple Grid Schemes for Solving the Euler Equations" AIAA Journal, Vol. 20, Nov. 1982, pp. 1565-1571.

63. Morton, K. W., and Paisley, M. F., "On the Cell-Centre and Cell-Volume Approaches to the Steady Euler Equations and the Use of Shock Fitting," Lecture Notes in Physics, Vol. 264, 1986, pp. 488-493.

64. Hall, M. G., and Fiddes, S. P., "Computational Fluid Dynamics in the United Kingdom ," AIAA Paper 87-1132, Jun. 1987.

65. Visbal, M. R., and Shang, J. S., "Comparative Study Between Two Navier-Stokes Algorithms for Transonic Airfoils," AlAA Journal, Vol. 24, Apr. 1986, pp. 599-606.

66. Mueller, B., "Navier-Stokes Solution for Hypersonic Flow over an Indented Nosetip ," AIAA Paper 85-1504, 1985.

67. Degani, D., and Steger, J. L., "Comparison Between Navier-Stokes and Thin-Layer Computations for Separated Supersonic Flow," AIAA Journal, Vol. 21, Nov. 1986, pp.1604-1606.

68. Pulliam, T. H., "Artificial Dissipation Models for the Euler Equations ," AIAA Journal, Vol. 21, Dec. 1986, pp. 1931-1940.

69. Takakura, Y. et al, "On the Recent Difference Schemes for the Three-Dimensional Euler Equations," AIAA Paper 87-1151, Jun. 1987.

70. Spekreijse, S. P., "Second Order Accurate Upwind Solutions of the 2D Steady Euler Equations by the Use of a Defect Correction Method," Lecture Notes in Mathematics, Vol. 1228, pp. 285-300., edited by Dold, A. et al.

71. Barth, T. J., "Anaysis of Implicit Local Linearization Techniques for Upwind and TVD Algorithms," AIAA Paper 87-0595, Jan. 1987. 
72. Shang, J. S., "Numerical Simulation of Wing-Fuselage Interference ," AIAA Journal, Vol. 20, Dec. 1982, pp. 1657-1658.

73. Jameson, A., "Numerical Solution of the Euler Equation for Compressible Inviscid Fluids ," Numerical Methods for the Euler Equations of Fluid Dynamics, edited by F. Angrand et al , SIAM, Philadelphia, 1985. , pp. 199-245.

74. McDonald, H., and Briley, W. R., "Computational Fluid Dynamic Aspects of Internal Flows," AIAA Paper 79-1445, 1979.

75. Turkel, E., "Acceleration to a Steady State for the Euler Equations ," Numerical Methods for the Euler Equations of Fluid Dynamics, edited by F. Angrand et al, SIAM, Philadelphia, 1985. , pp. 281-311.

76. Thomas, J. L., van Leer, B., and Walters, R. W., "Implicit Flux Split Schemes for the Euler Equations," AIAA Paper 85-1680, Jul. 1985.

77. Schroeder, W., and Haenel, D., "An Unfactored Implicit Scheme with Multigrid Acceleration for the Solution of the Navier-Stokes Equations," Computer \& Fluids, Vol. 15, No. 3, 1987, pp. 313-336.

78. Rudy, D. H., and Strikwerda, J. C., "Boundary Conditions for Subsonic Compressible Navier-Stokes Calculations," Computers and Fluids, Vol. 9, 1981, pp.327-338.

79. White, F. M., Viscous Fluid Flow, McGraw Hill, Inc., 1974.

80. Haenel, D., Schwane, R., and Seider, G., "On the Accuracy of Upwind Schemes for the Solution of the Navier- Stokes Equations," AIAA Paper 87-1105, Jun. 1987.

81. Moretti, G., "Importance of Boundary Conditions in the Numerical Treatment of Hyperbolic Equations," The Physics of Fluids, Supplement II, Sep. 1969, pp. II-13-II-20.

82. Moretti, G., "Comment on "Stability Aspects of Diverging Subsonic Flows "," AlAA Journal, Vol. 19, May 1981, p. 669.

83. Moretti, G., "Experiments on Initial and Boundary conditions ," Numerical and Physical Aspects of Aerodynamic Flows, edited by Cebeci, T. , Springer Verlag 1982.

84. Moretti, G., and Pandolfi, M., "Critical Study of Calculations of Subsonic Flows in Ducts," AIAA Journal, Vol. 19, Apr. 1981, pp. 449-457. 
85. Yee, H. C., Beam, R. M., and Warming, R. F., "Boundary Approximations for Implicit Schemes for One-Dimensional Inviscid Equations of Gasdynamics," AIAA Journal, Vol. 20, Sep. 1982, pp. 1203-1211.

86. Shang, J. S., "An Assessment of Numerical Solutions of the Compressible Navier- Stokes Equations," AIAA Paper 84-1549 , Jun. 1984.

87. Gustafsson, B. and Sundstroem, A., "Incompletely Parabolic Problems in Fluid Dynamics," SIAM Journal of Applied Mathematics Vol. 35, No. 2, 1978, pp. 343-357.

88. Rudy, D. H. and Strikwerda, J. C., "A Nonreflecting Outflow Boundary Conditions for Subsonic Navier-Stokes Calculations ," Journal of Computational Physics Vol. 36, 1980, pp. 71-92.

89. Roache, P. J., Computational Fluid Dynamics, Revised printing,Hermos Publishers, 1976.

90. Hung, C. M. and MacCormack , R. W., "A Numerical Solutions of Supersonic and Hypersonic Laminar Compression Corner Flows," AIAA Paper 75-2, Jan. 1975.

91. Roberts, G. O., "Computational Meshes for Boundary Layer Flows," Proc. of 2nd International Conference on Numerical Methods in Fluid Dynamics, edited by J. Ehlers et al, Sep. 1970.

92. Cline, M. C., "VNAP2: A Computer Program for Computation of Two-dimensional , Time-dependent, Compressible, Turbulent Flow," Los Alamos National Labaratory Report LA-8872, Aug. 1981.

93. Hakkinen, R.J., Greber, I., Trilling, L., and Abarnel, S. S., "The Interaction of an Oblique Shock Wave with a Laminar Boundary Layer," Technical Report 57-1, Fluid Dynamic Research Group, M.I.T. , May 1957.

94. MacCormack, R. W. , "An Efficient Numerical Method for Solving the Time Dependent Compressible Navier-Stokes Equations at High Reynolds Number ," Computing in Applied Mathematics, AMD Vol. 18, Dec. 1976, pp. 49-64.

95. Li, C. P., "A Numerical Study of Separated Flows Induced by Shock-Wave /Boundary Layer Interaction," AIAA Paper 77-168, Jan. 1977. 
96. Shang, J. S., "An Implicit-Explicit Method for Solving the Navier-Stokes Equations ," AIAA Paper 77-646, 1977.

97. Hama, F. R., "Experimental Studies on the Lip Shock," AIAA Journal, Vol. 6, Feb. 1968, pp. 212-219.

98. Munz, C. D., "On the Comparison and Construction of Two-Step Schemes for the Euler Equations,"

99. Hollanders, H., and Viviand, H., "The Numerical Treatment of Compressible High Reynolds Number Flows," Computational Fluid Dynamics, Vol. 2, 1980, pp. 1-66, edited by Kollmann, W. 
Figures 


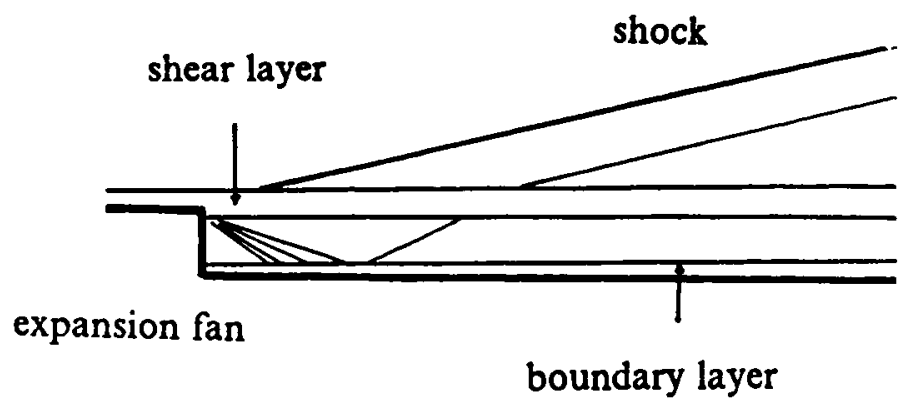

a) supersonic injection (without wedge ).

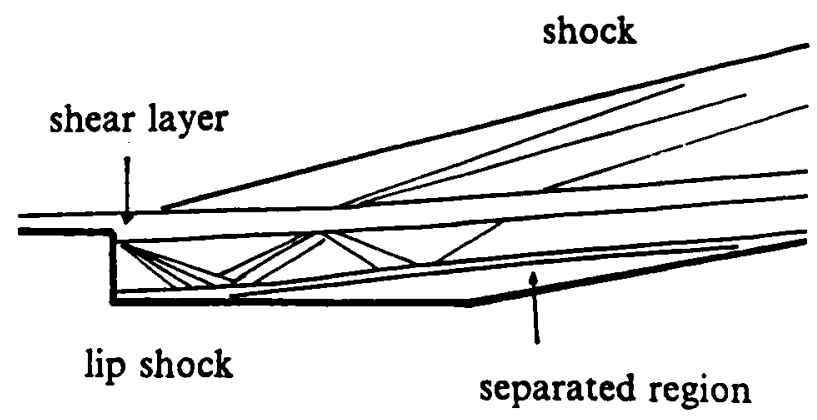

b) supersonic injection over a 10 degree wedge.

Figure 1. Schematic representation of supersonic slot injection problems. 


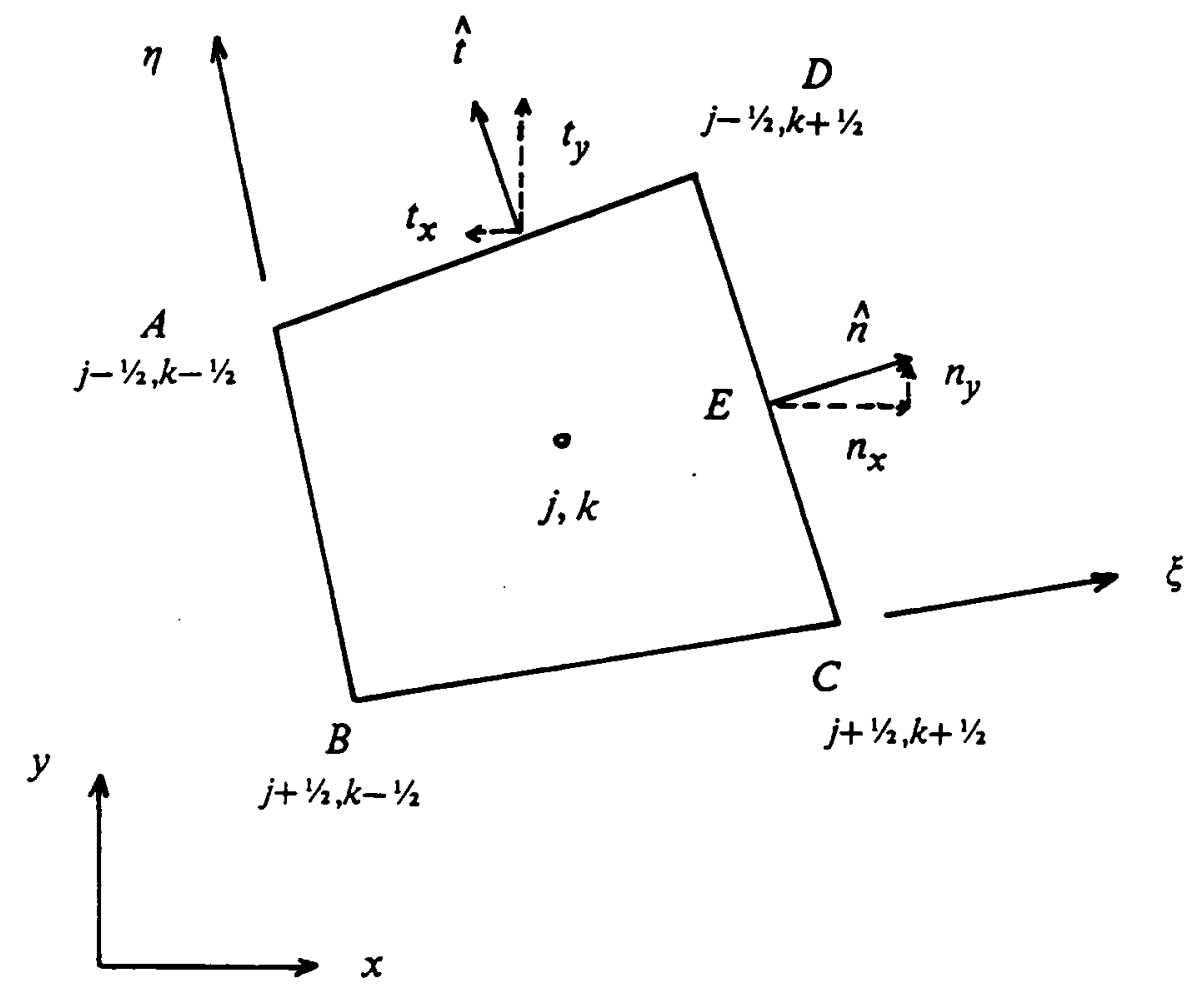

Figure 2. A finite volume element. 
upper boundary

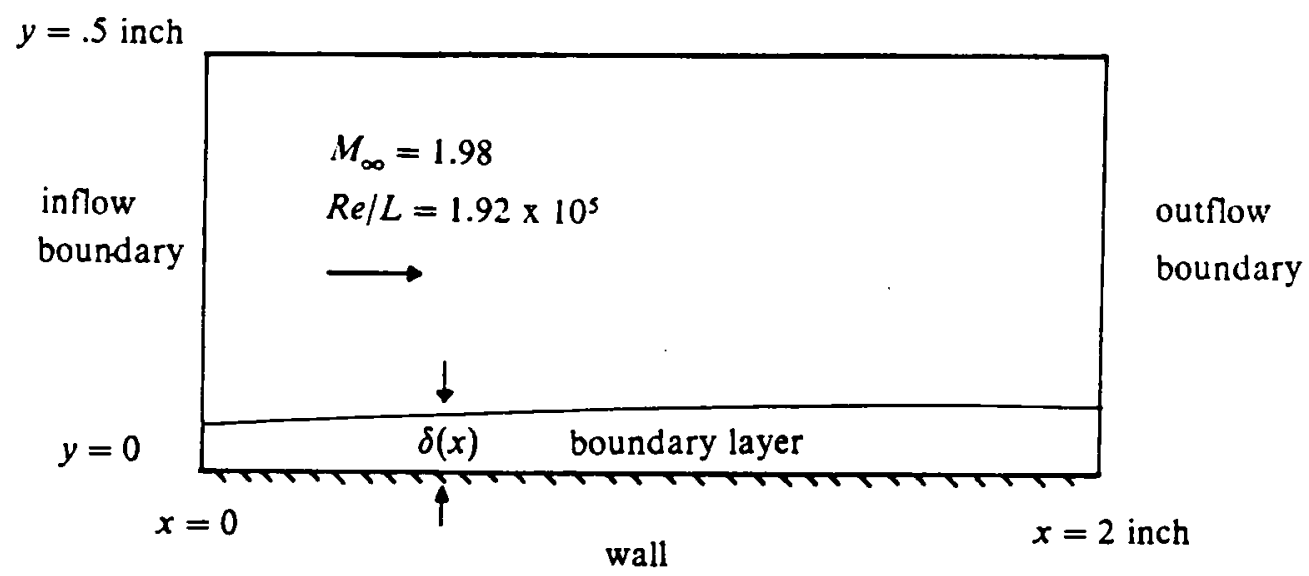

Figure 3. Schematic of compressible laminar boundary layer problem. 


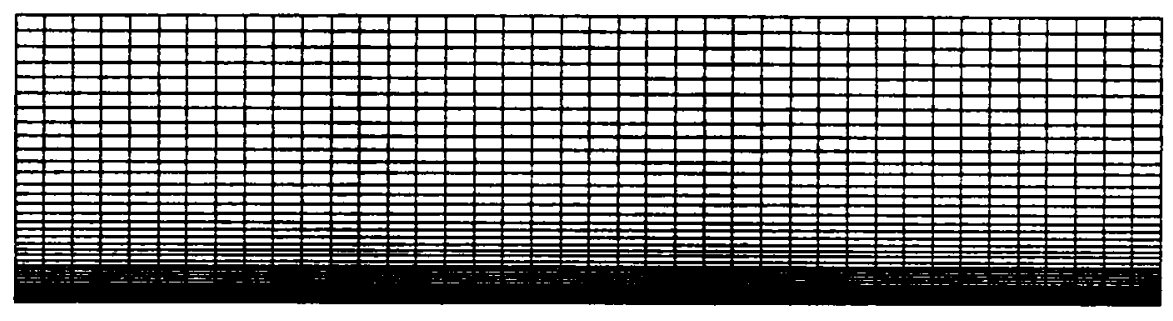

Figure 4. $41 \times 41$ grid for compressible laminar boundary layer problem. 


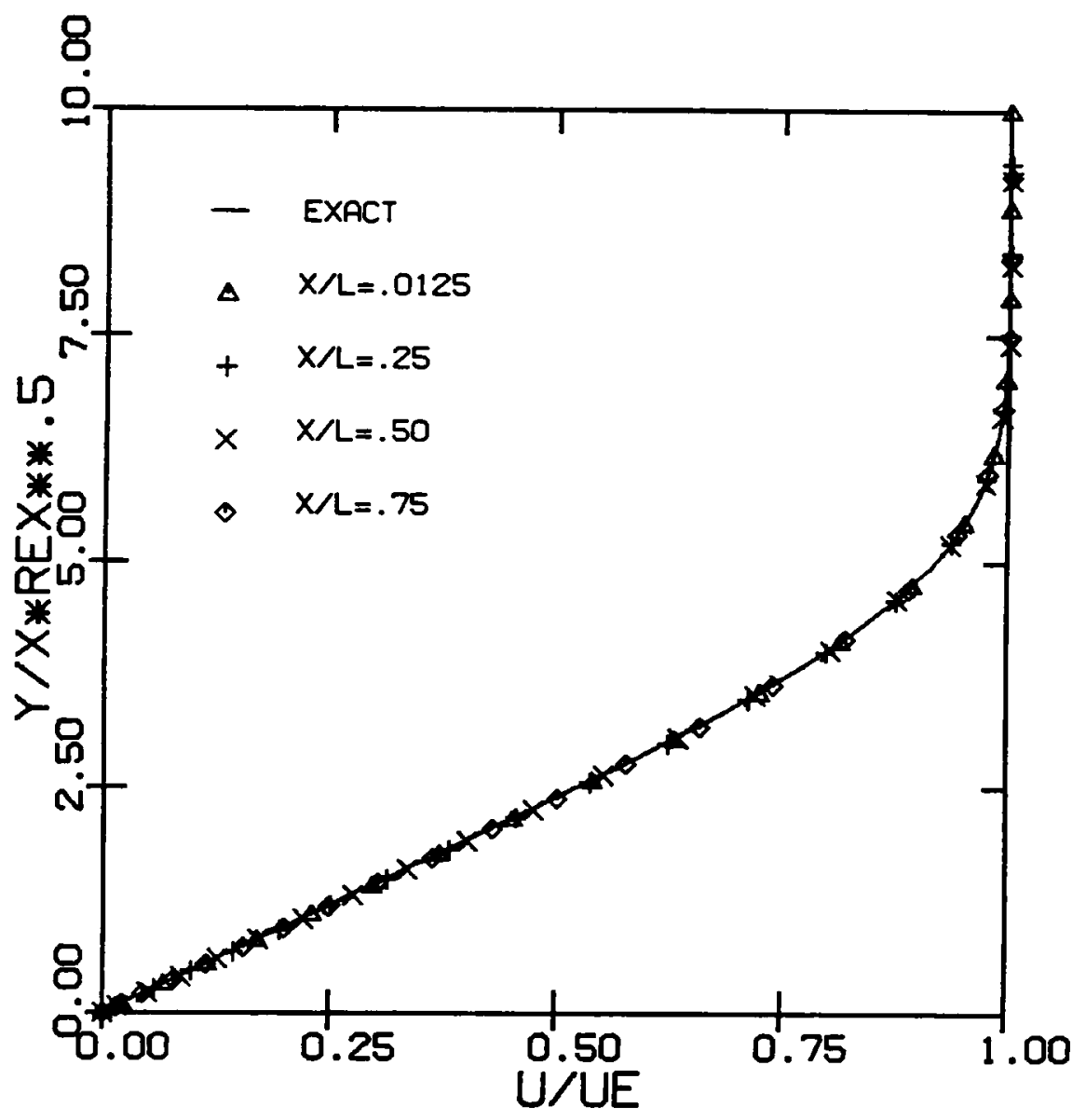

Figure 5. Velocity profiles in the boundary layer, $41 \times 41$ grid (FDS-Roe). 


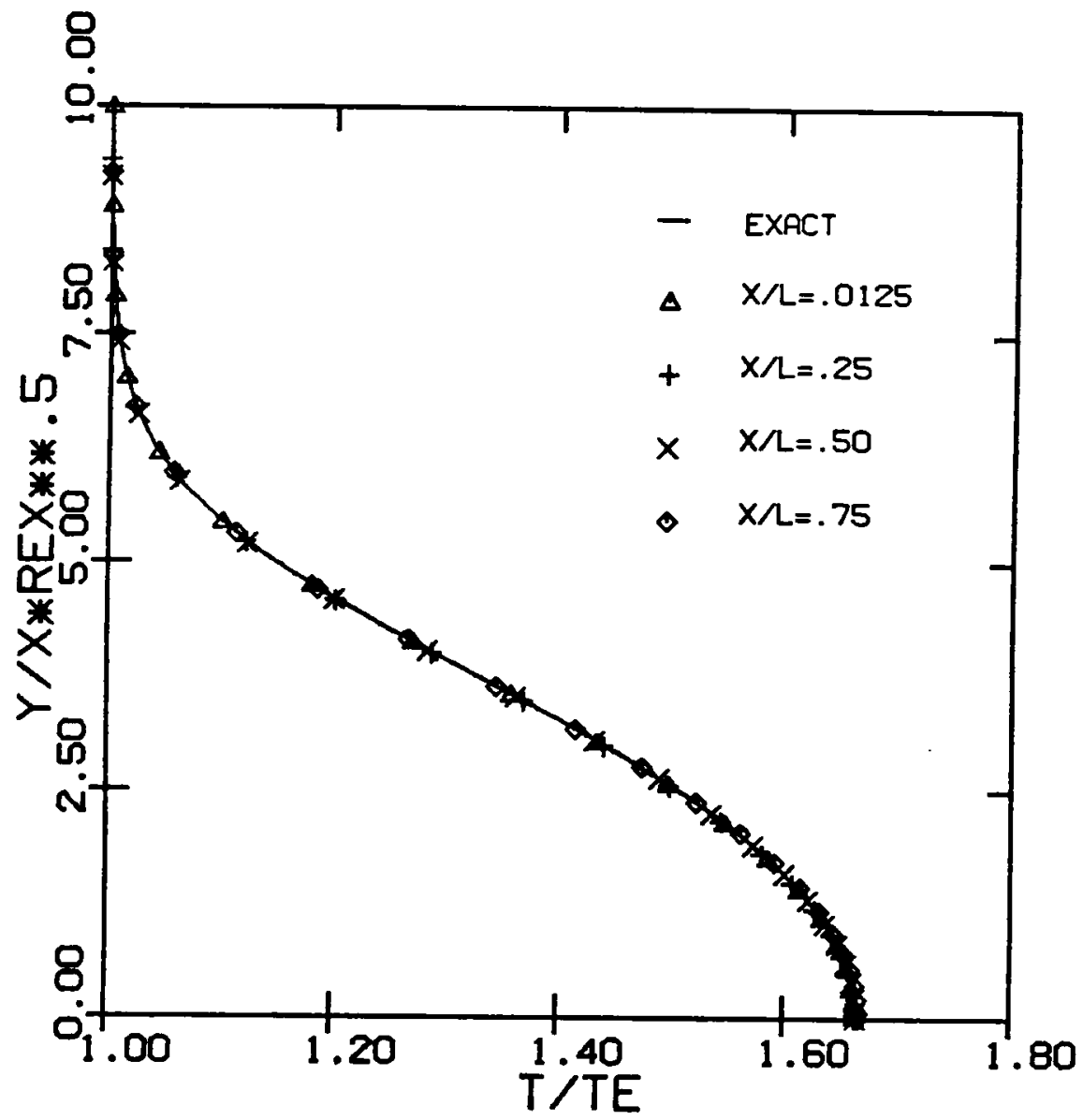

Figure 6. Temperature profiles in the boundary layer, $41 \times 41$ grid (FDS-Roe). 


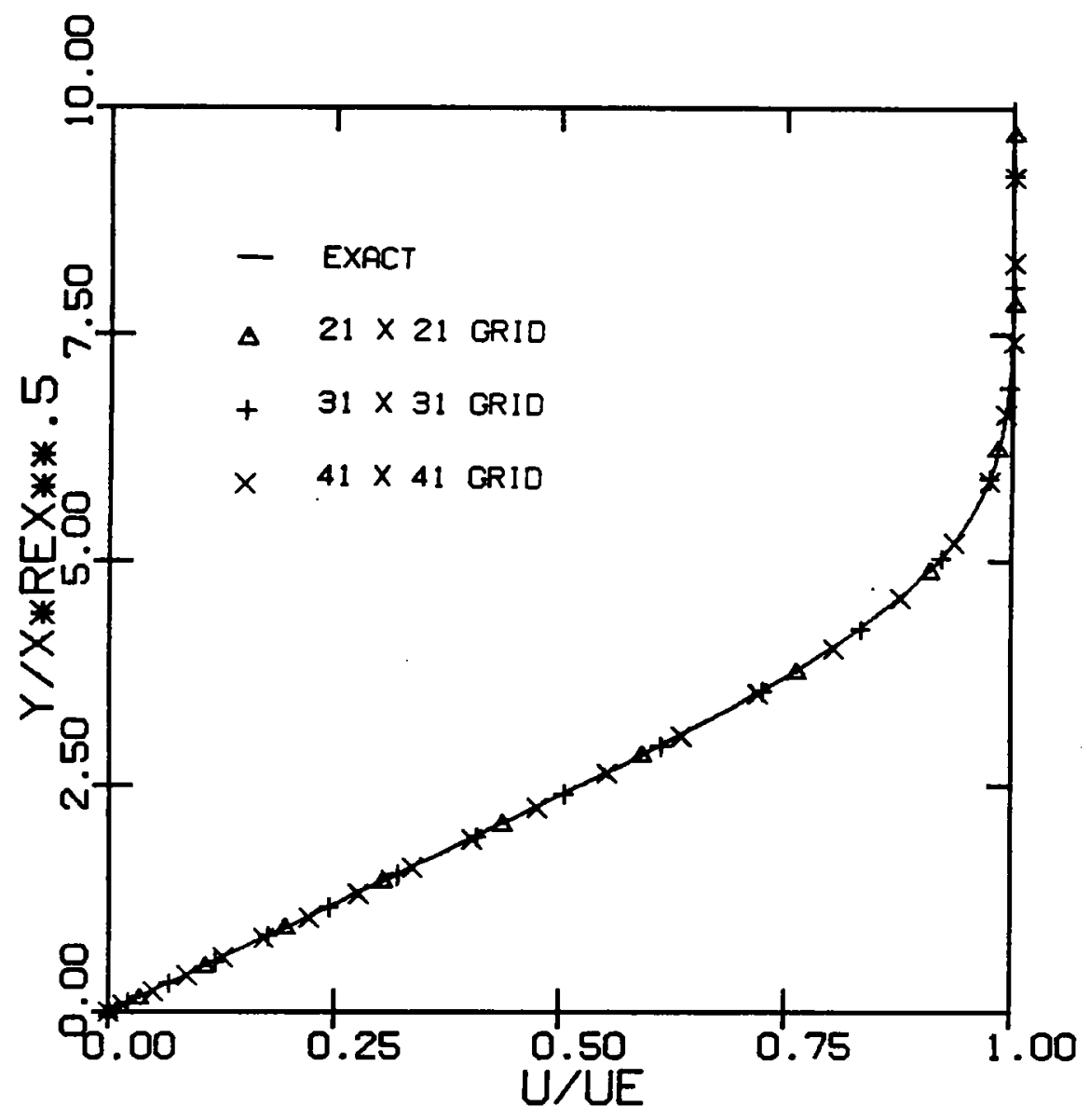

Figure 7. The effect of grid refinement, $\mathrm{X} / \mathrm{L}=.5$ - velocity (FDS-Roe). 


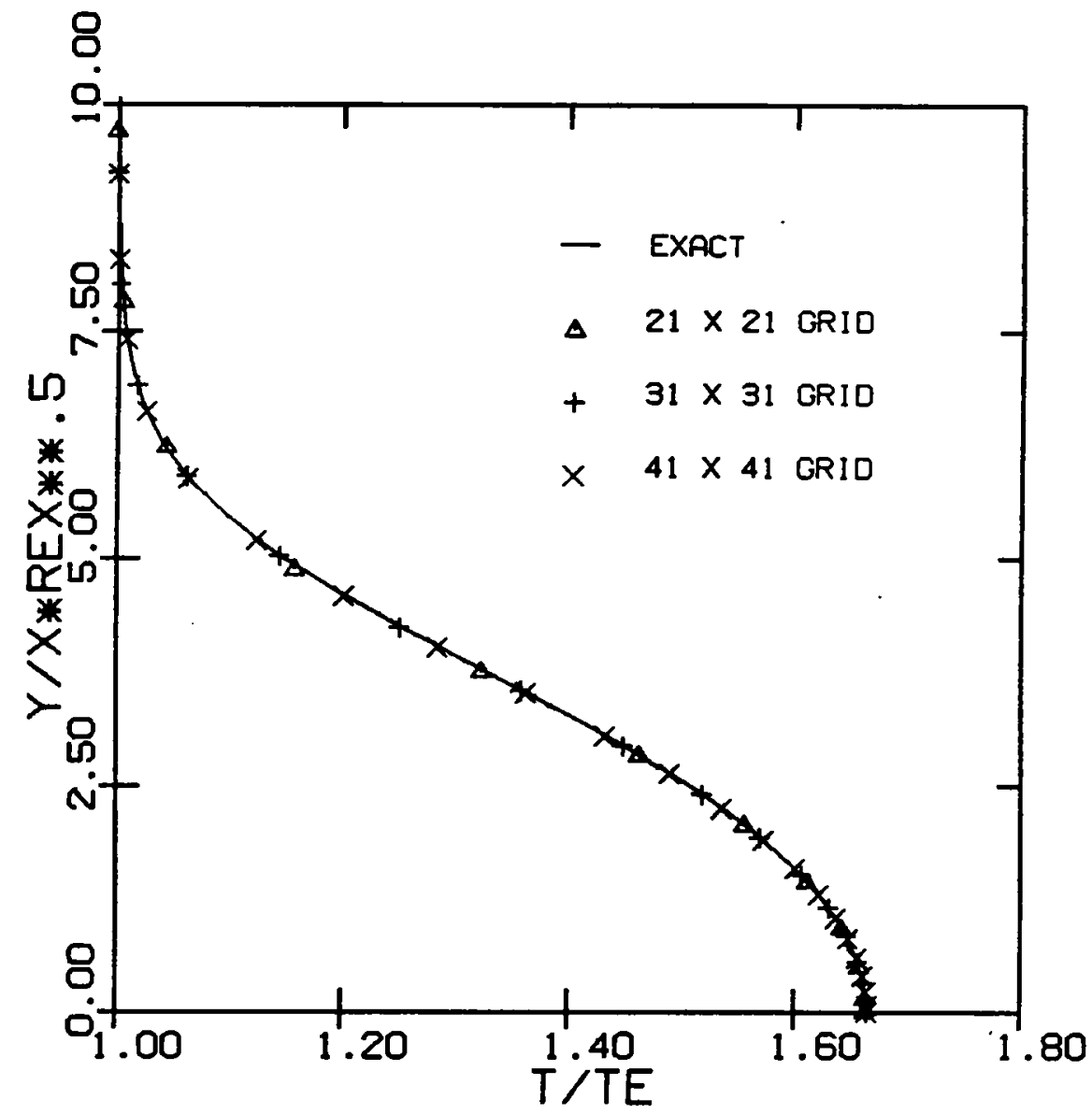

Figure 8. The effect of grid refinement, $\mathrm{X} / \mathrm{L}=.5$ - temperature (FDS-Roe). 


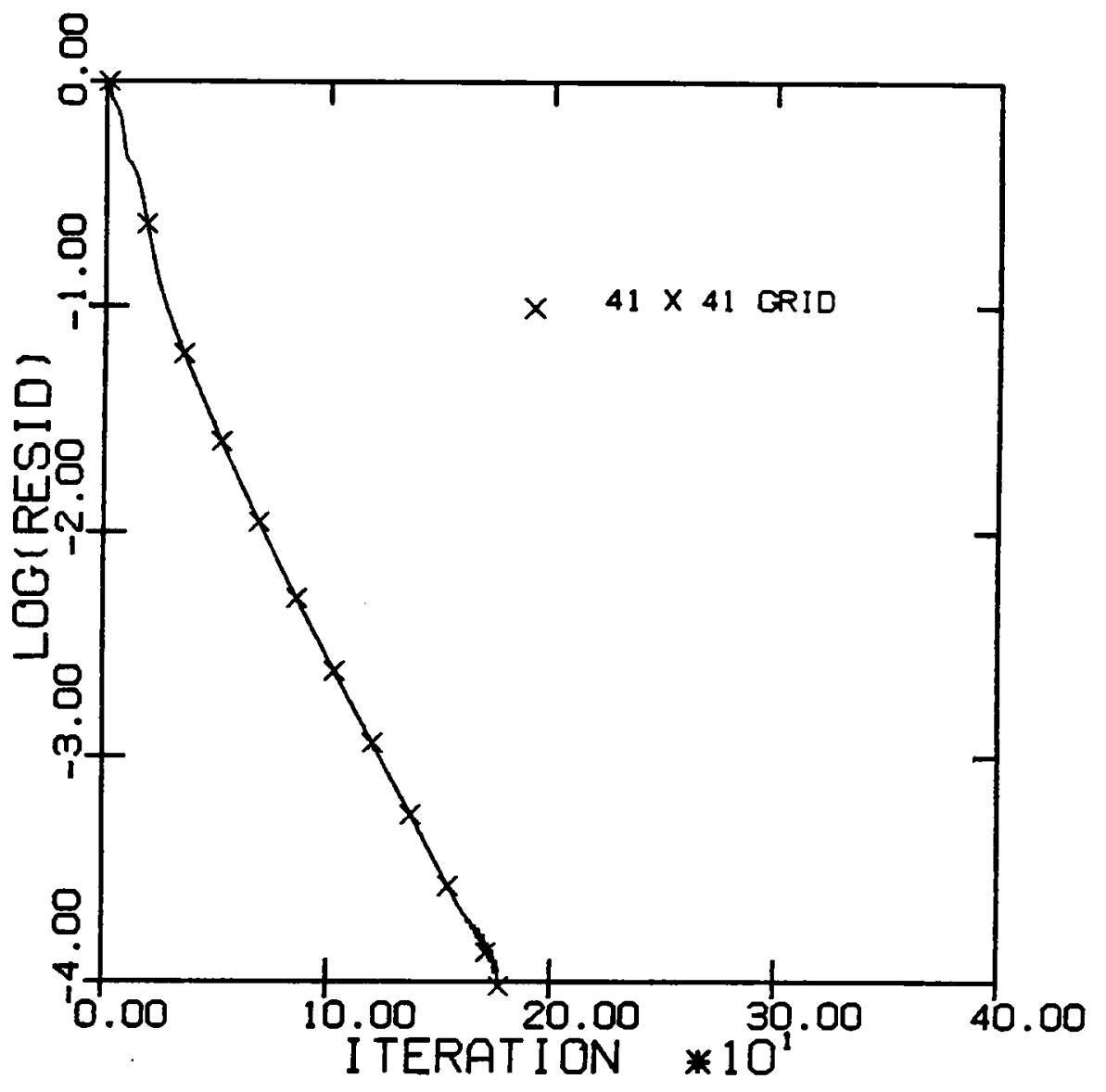

Figure 9. Convergence history for the compressible laminar boundary layer, $41 \times 41$ grid (FDS-Roe). 


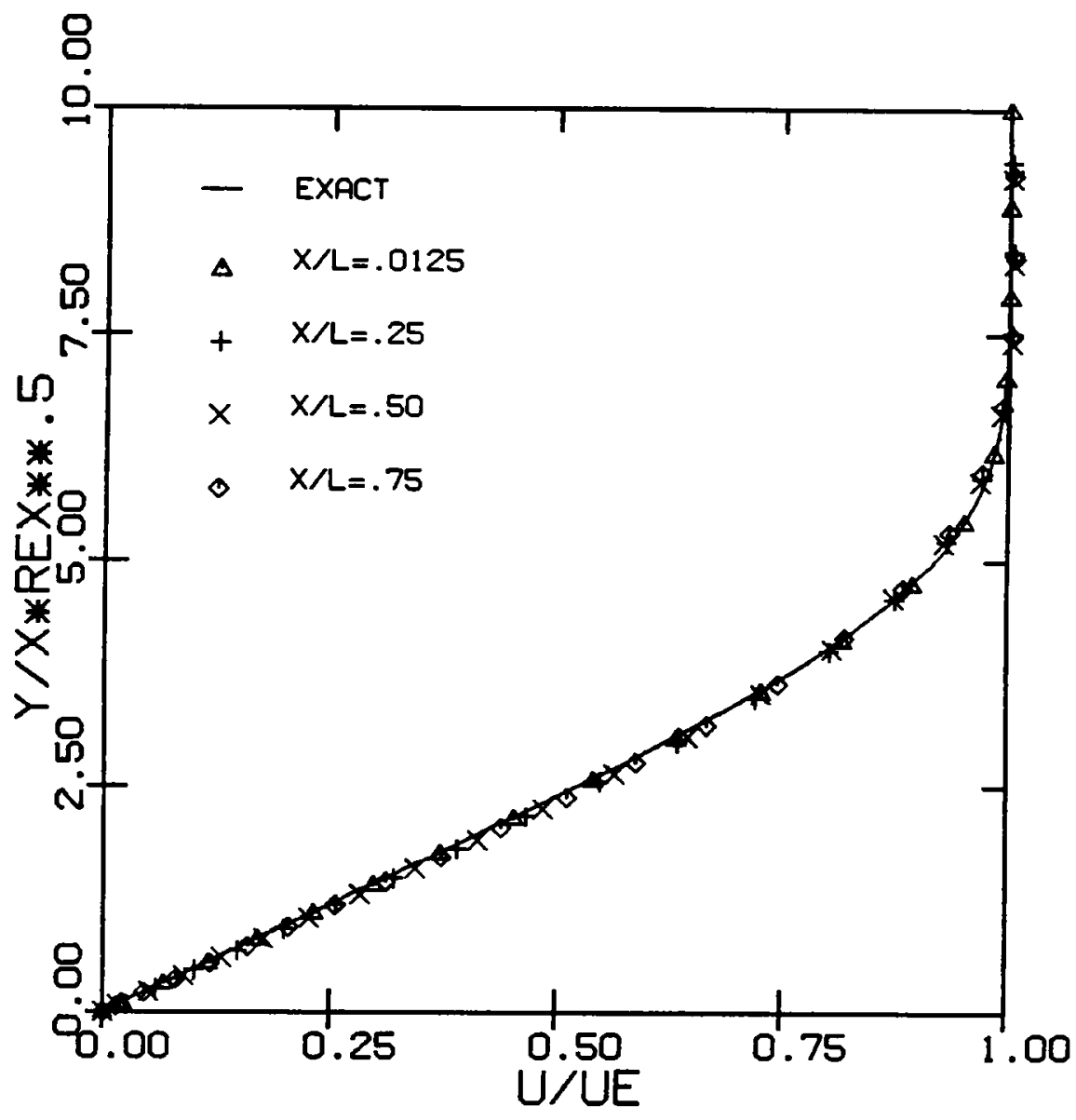

Figure 10. Velocity profiles in the boundary layer, $41 \times 41$ grid (FVS-van Leer). 


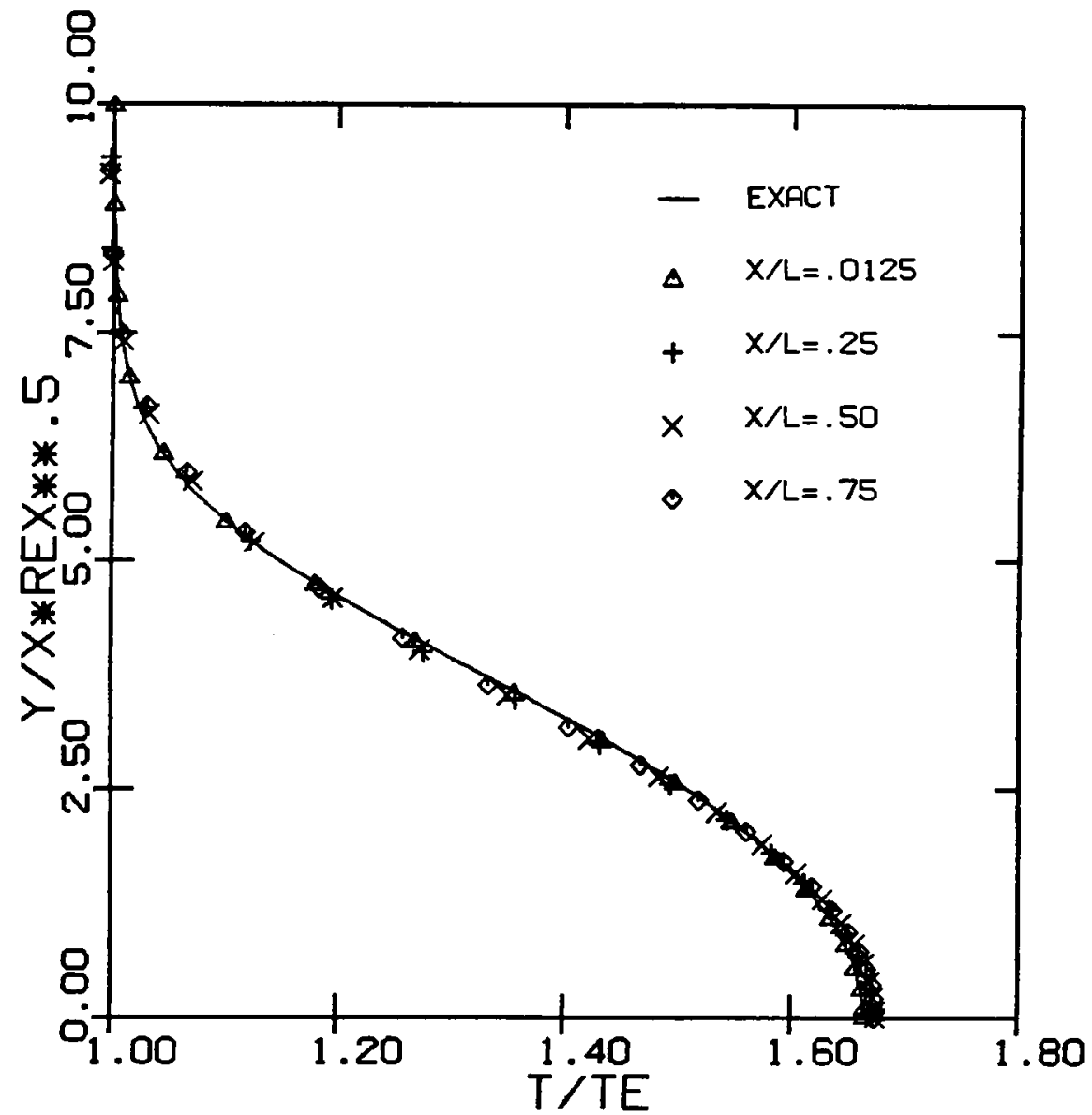

Figure 11. Temperature profiles in the boundary layer, $41 \times 41$ grid (FVS-van Leer). 


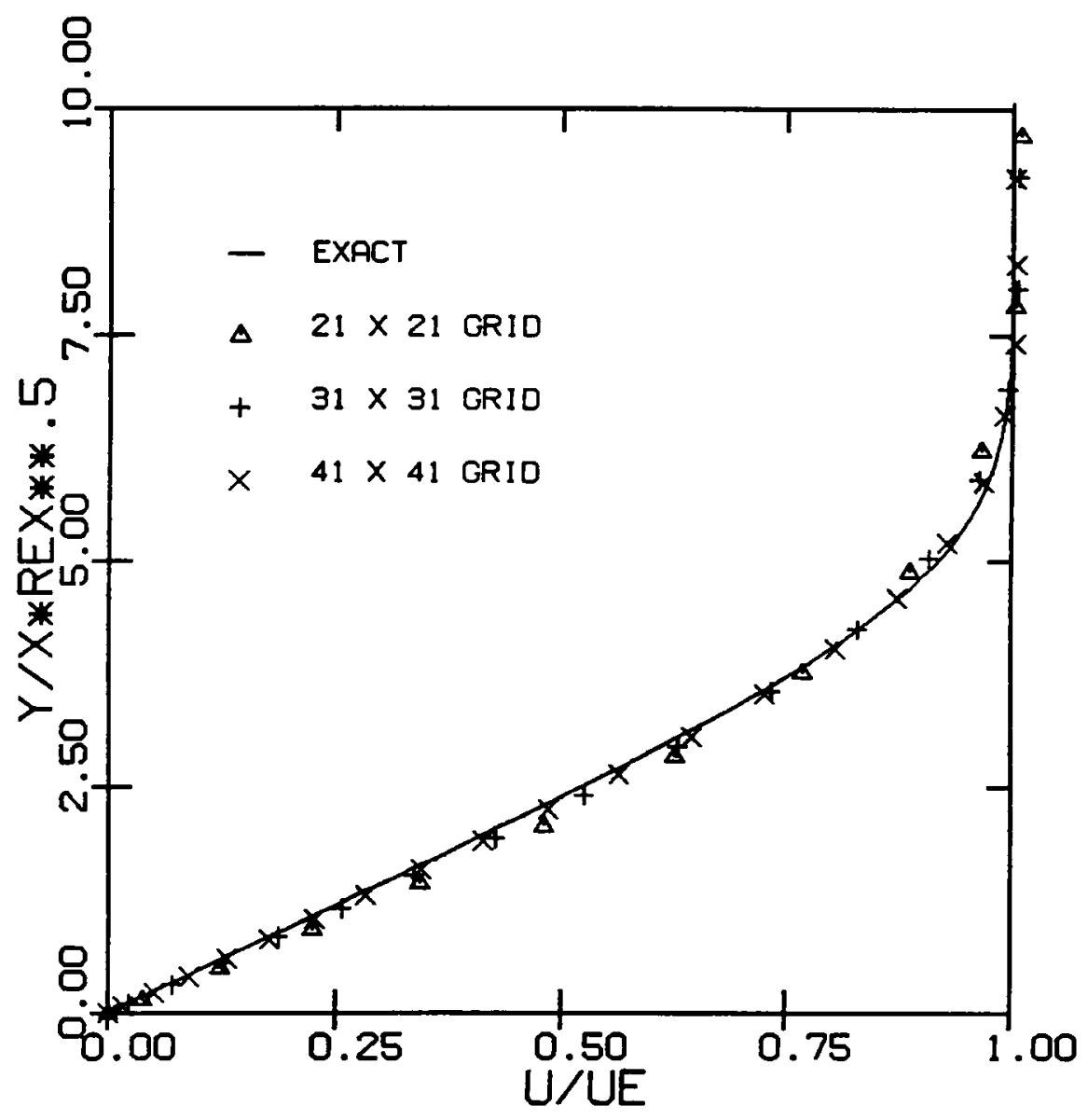

Figure 12. The effect of grid refinement, $\mathrm{X} / \mathrm{L}=.5$ - velocity (FVS-van Leer). 


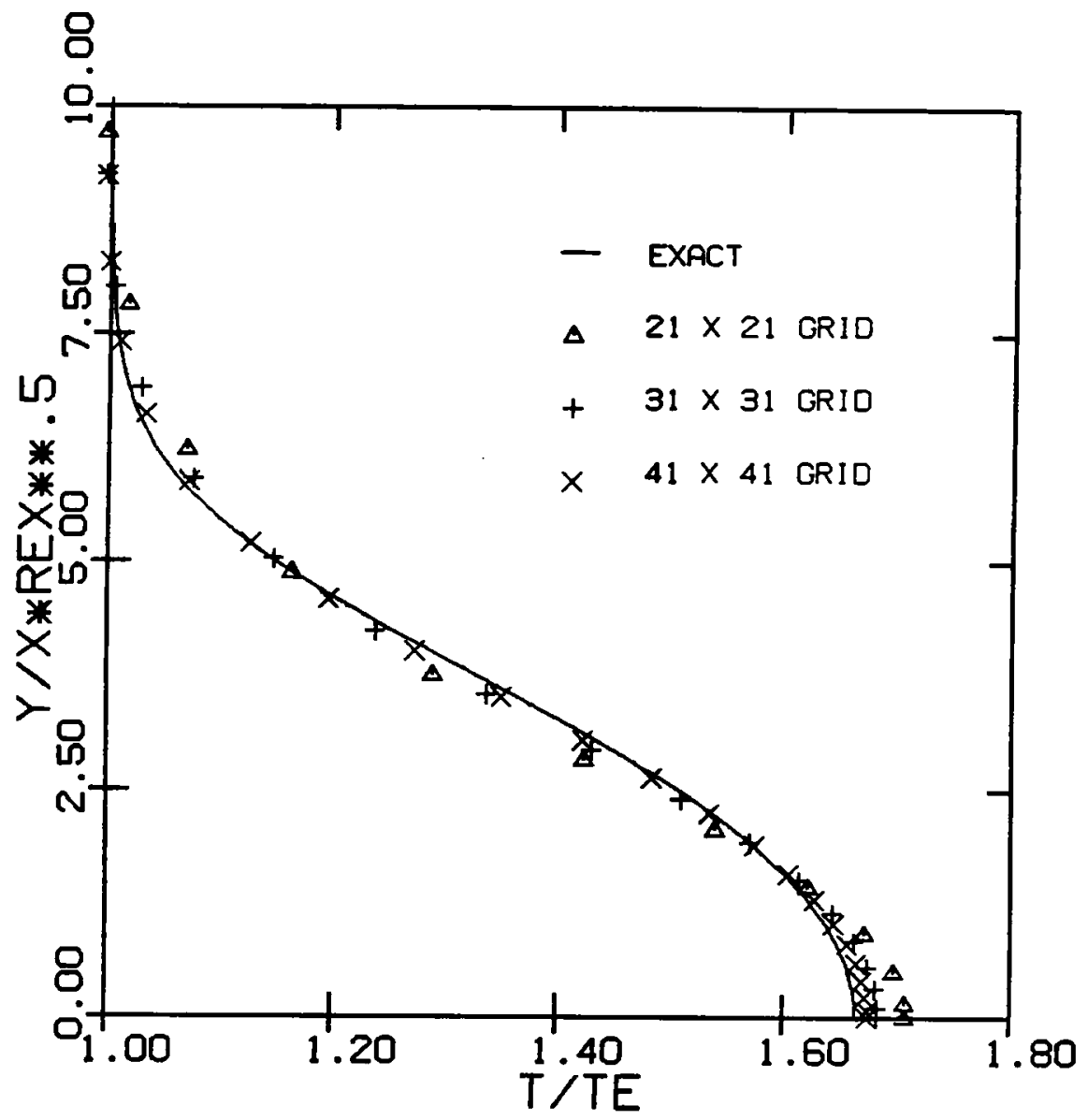

Figure 13. The effect of grid refinement, $\mathrm{X} / \mathrm{L}=.5$ - temperature (FVS-van Leer). 


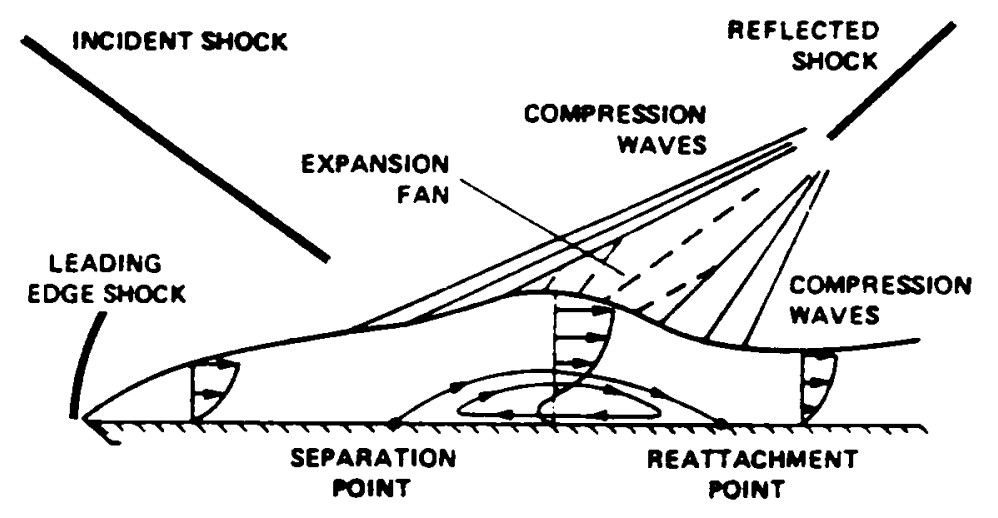

Figure 14. A sketch of laminar boundary layer-shock interaction problem( from MacCormack and Baldwin). 


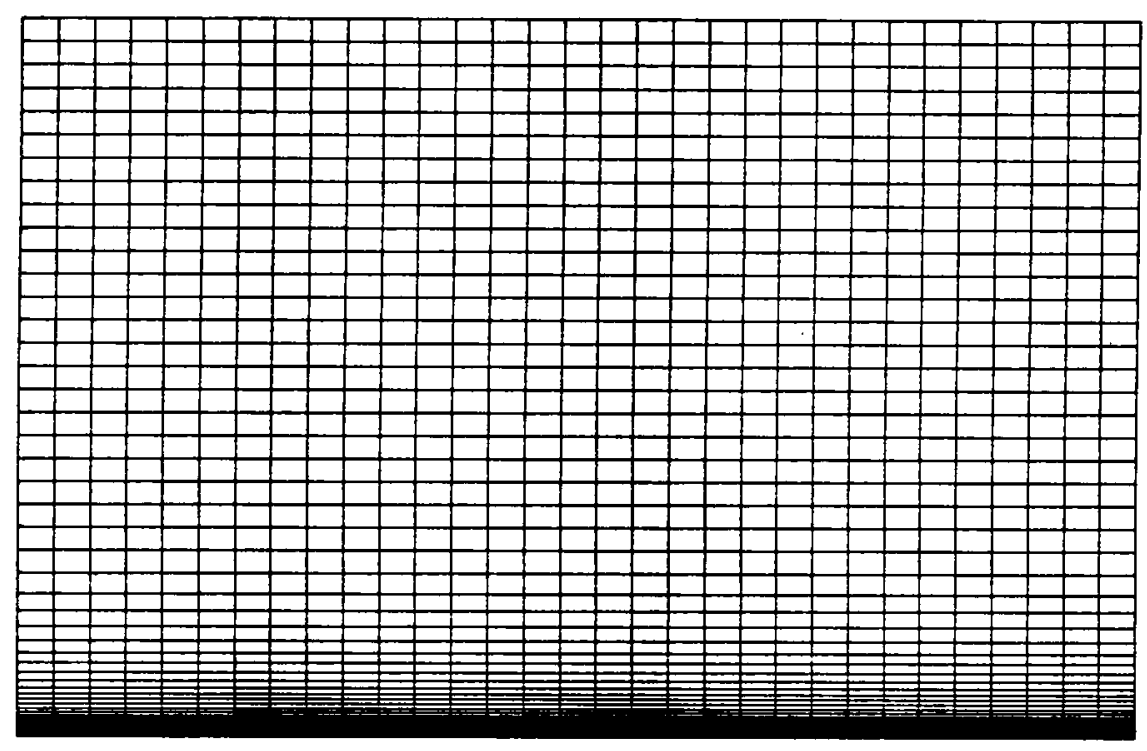

Figure 15. $32 \times 57$ grid for laminar boundary layer-shock interaction problem. 


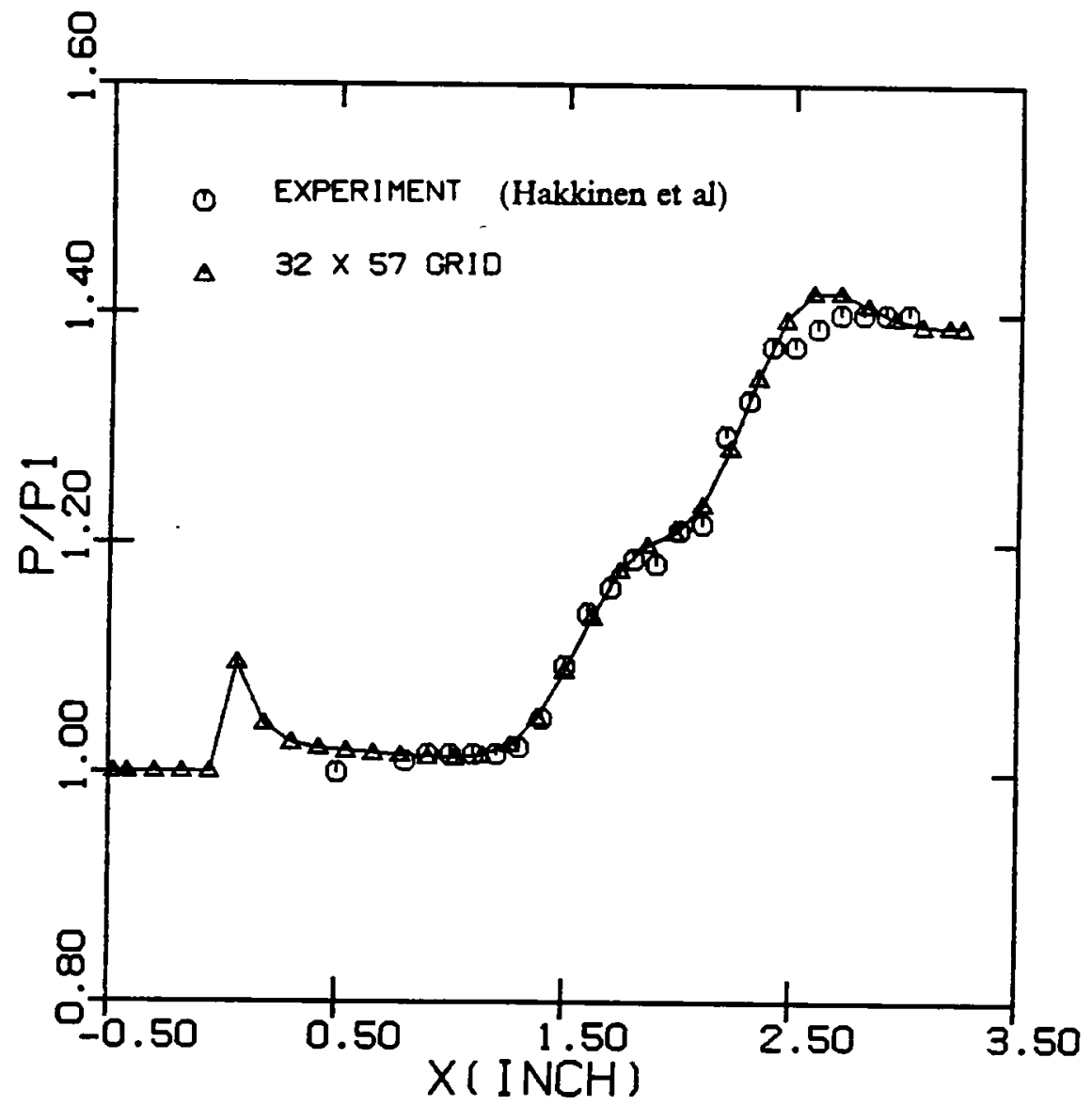

Figure 16. Wall pressure distribution for the laminar boundary layer shock interaction problem (FDS-Roe). 


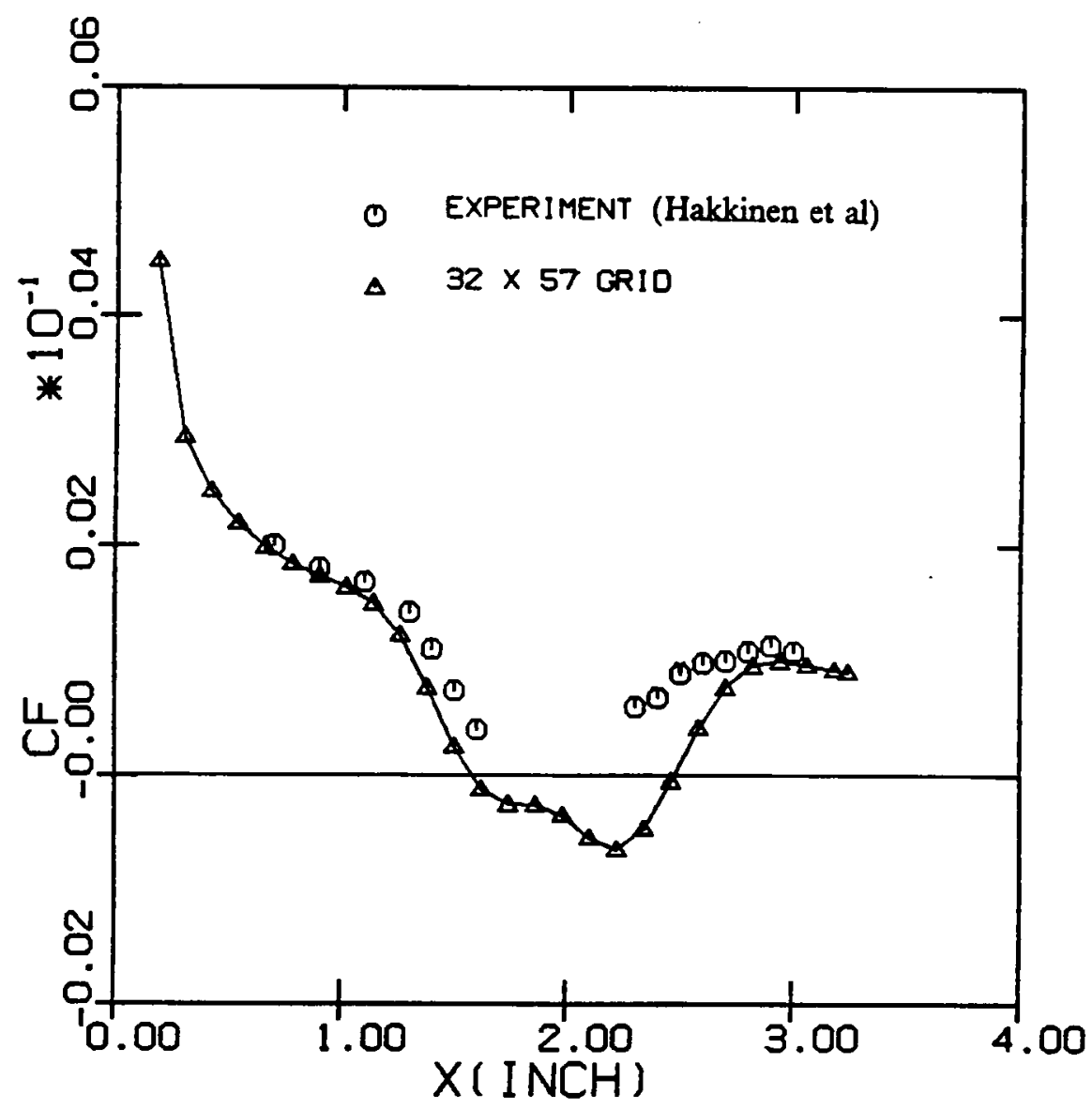

Figure 17. Wall skin friction coefficient distribution for the laminar boundary layer - shock interaction problem (FDS-Roe). 


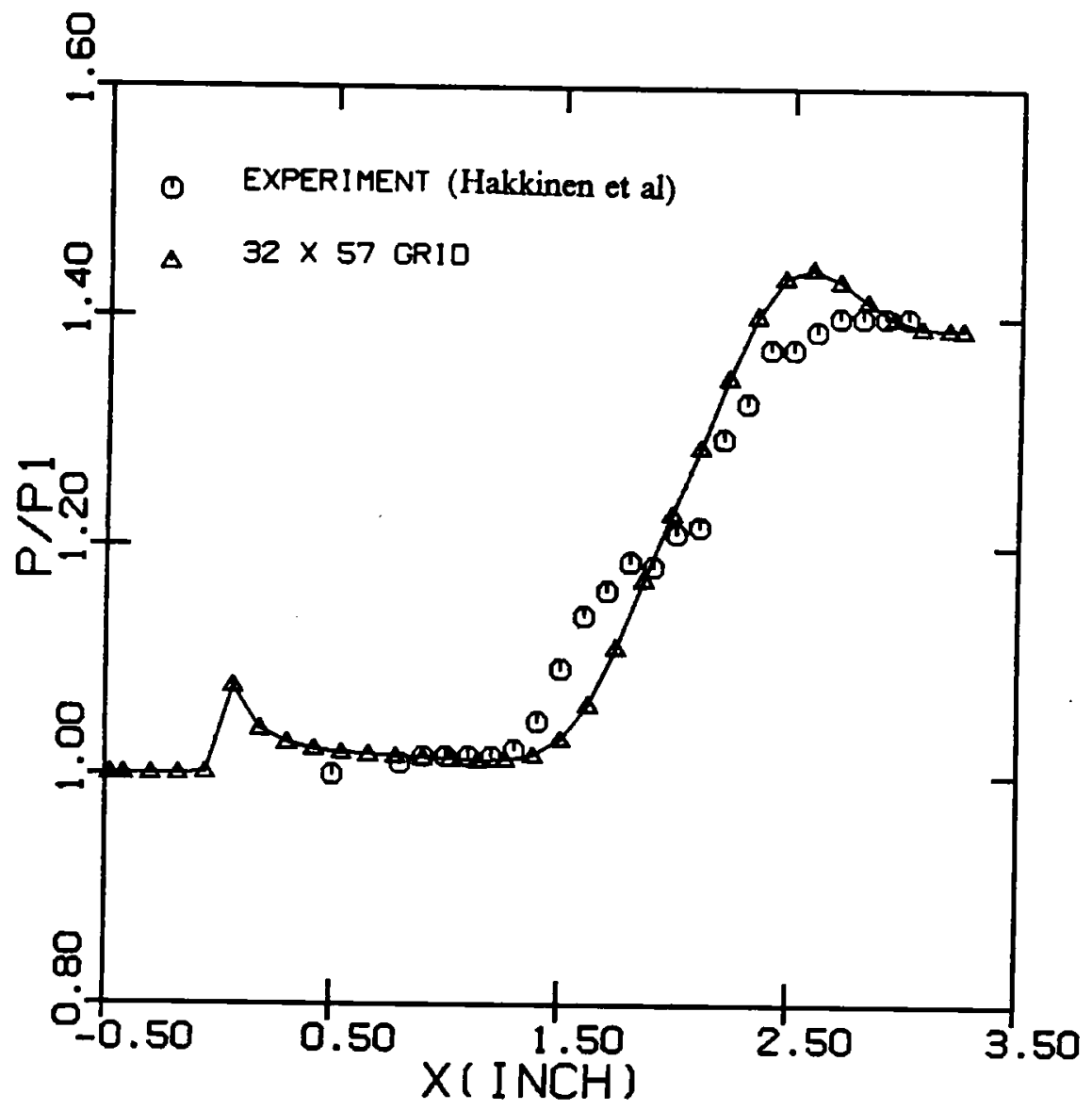

Figure 18. Wall pressure distribution for the laminar boundary layer shock interaction problem (FVS-van Leer). 


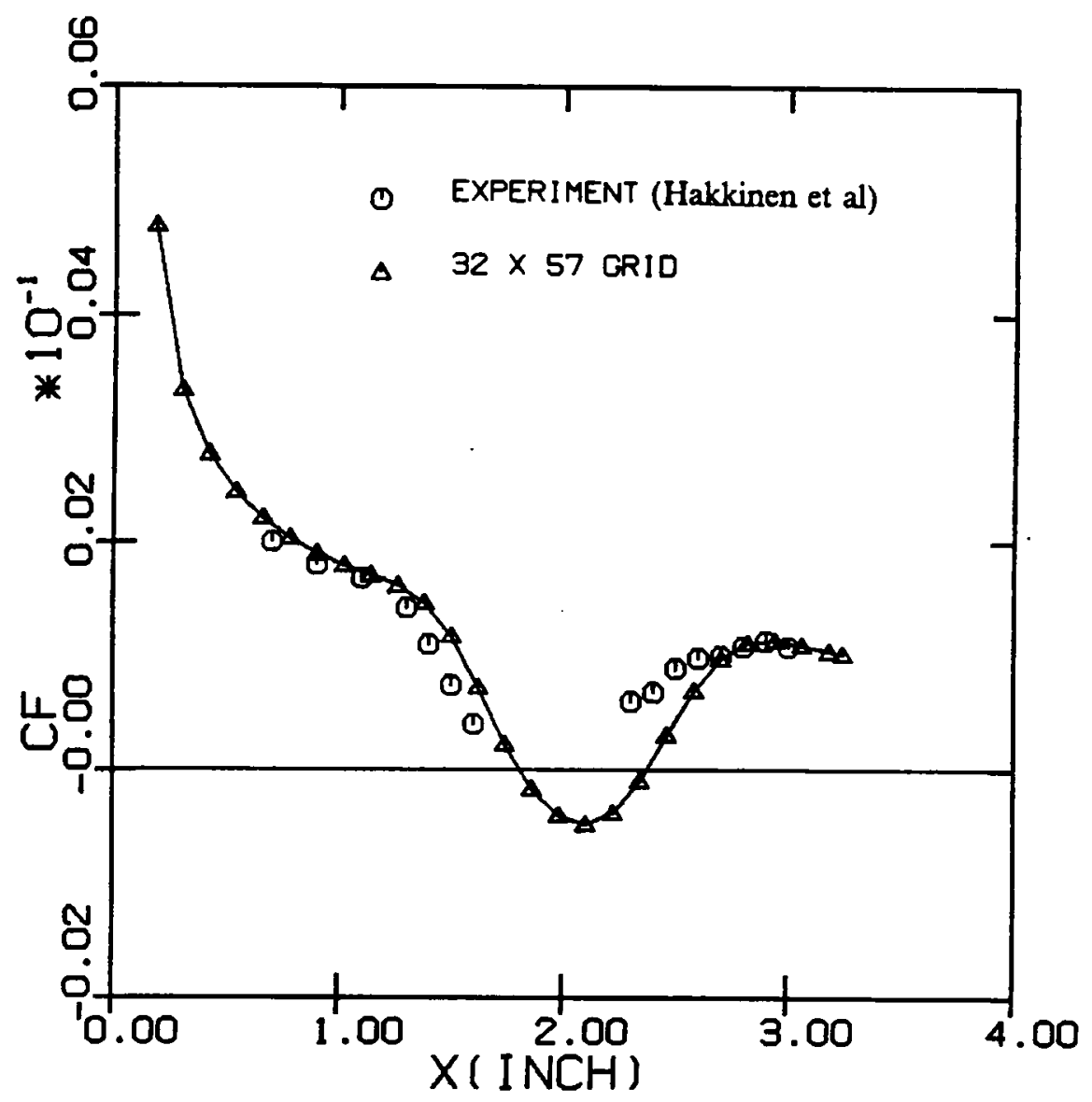

Figure 19. Wall skin friction coefficient distribution for the laminar boundary layer - shock interaction problem (FVS-van Leer). 


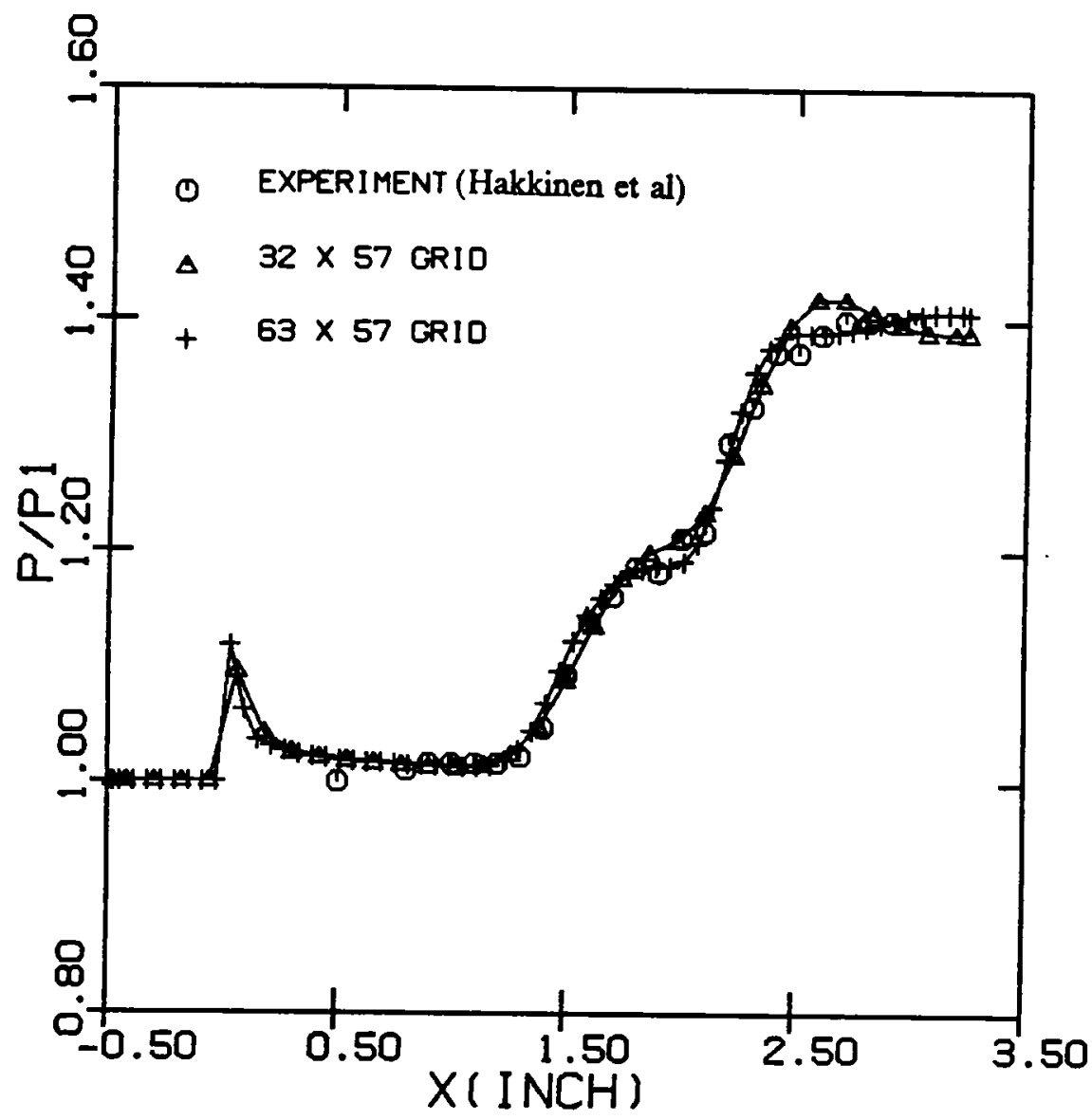

Figure 20. Grid refinement for the laminar boundary layer - shock interaction, wall pressure (FDS-Roe). 


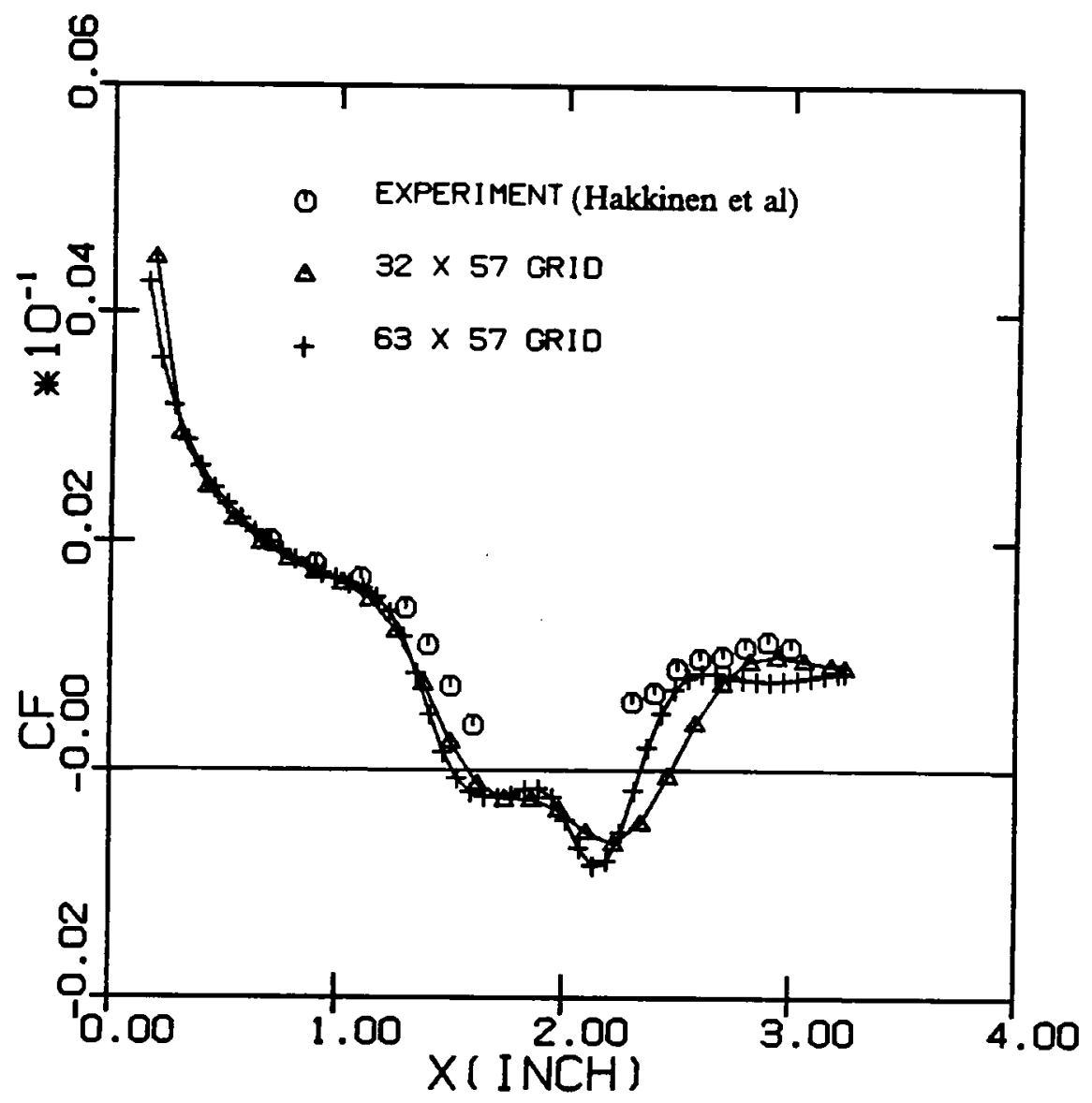

Figure 21. Grid refinement for the laminar boundary layer - shock interaction, skin friction (FDS-Roe). 


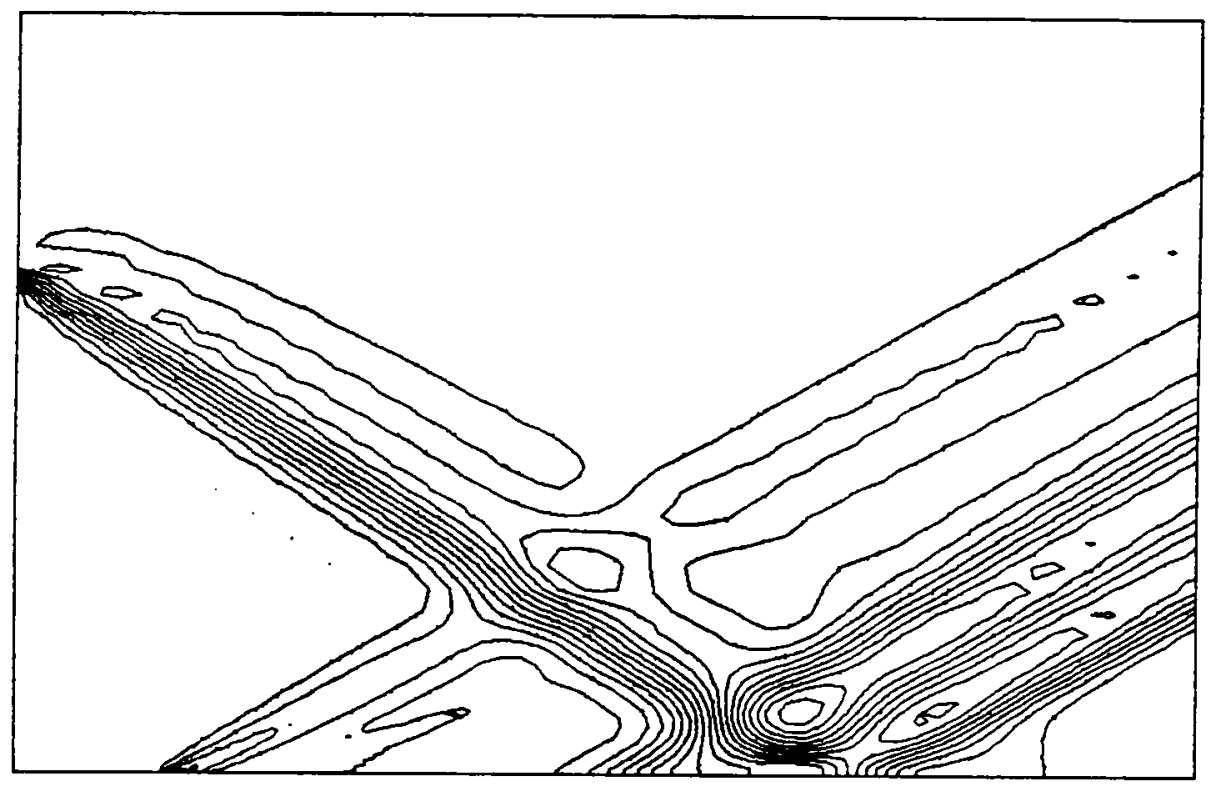

Figure 22. Pressure contour for the laminar boundary layer - shock interaction, $63 \times 57$ grid (FDS-Roe). 


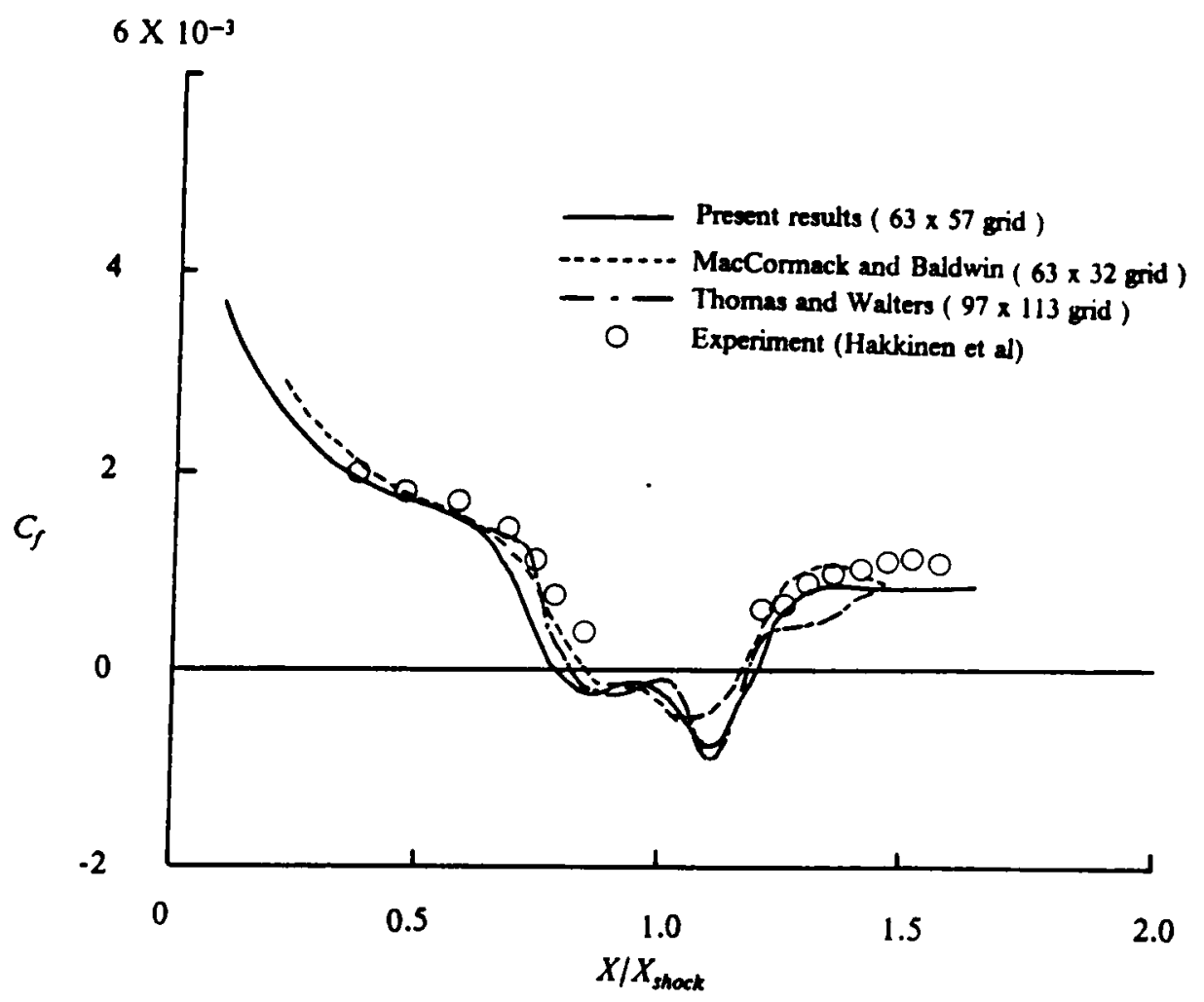

Figure 23. Comparison of computations with experiment for the laminar boundary layer - shock interaction problem. 


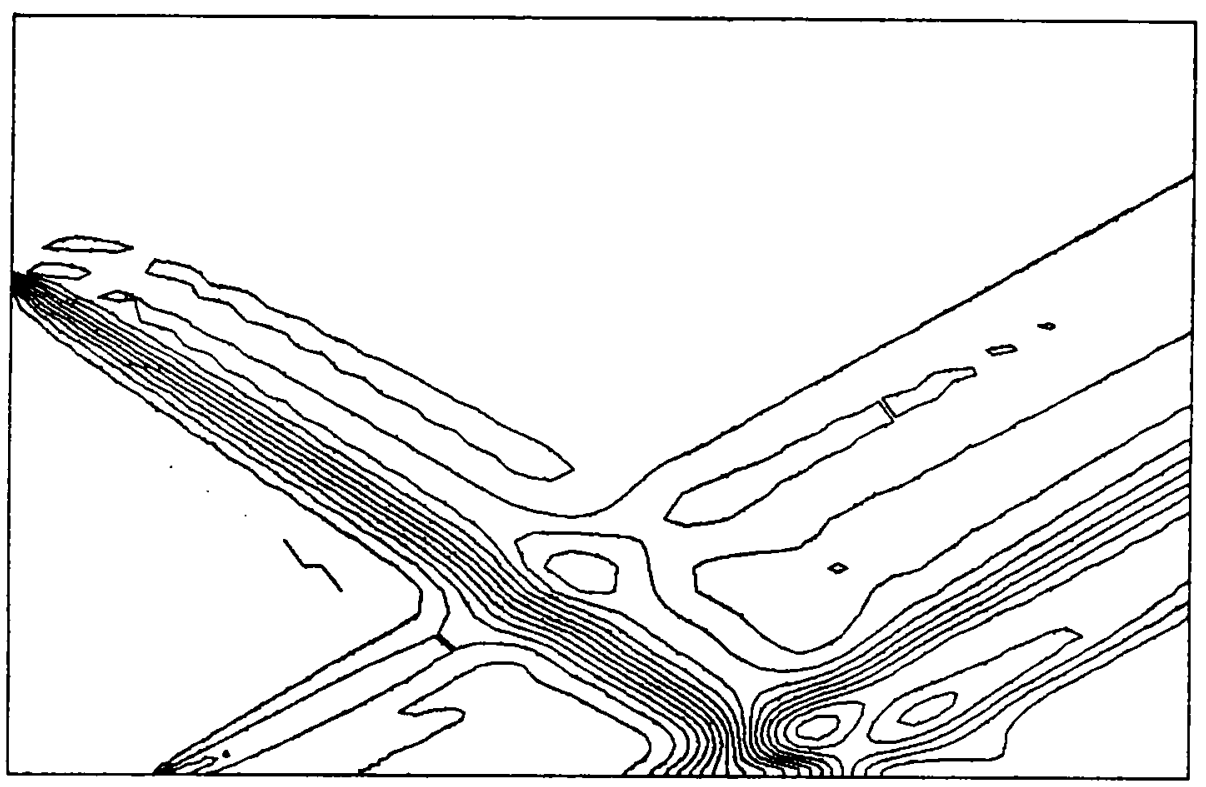

Figure 24. Pressure contour for the laminar boundary layer - shock interaction, $63 \times 57$ grid (FVS-van Leer). 


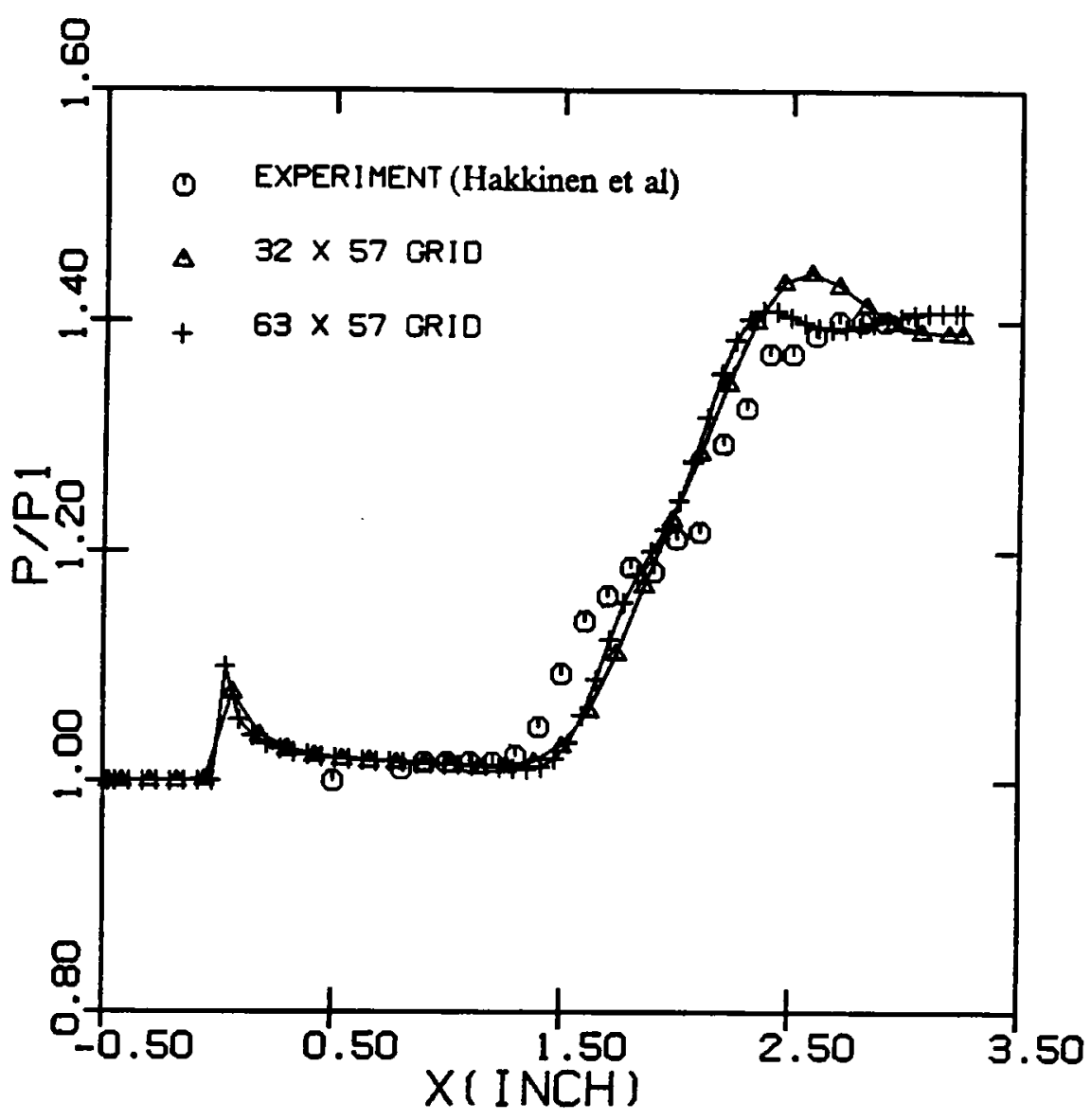

Figure 25. Grid refinement for the laminar boundary layer - shock interaction, wall pressure (FVS-van Leer). 


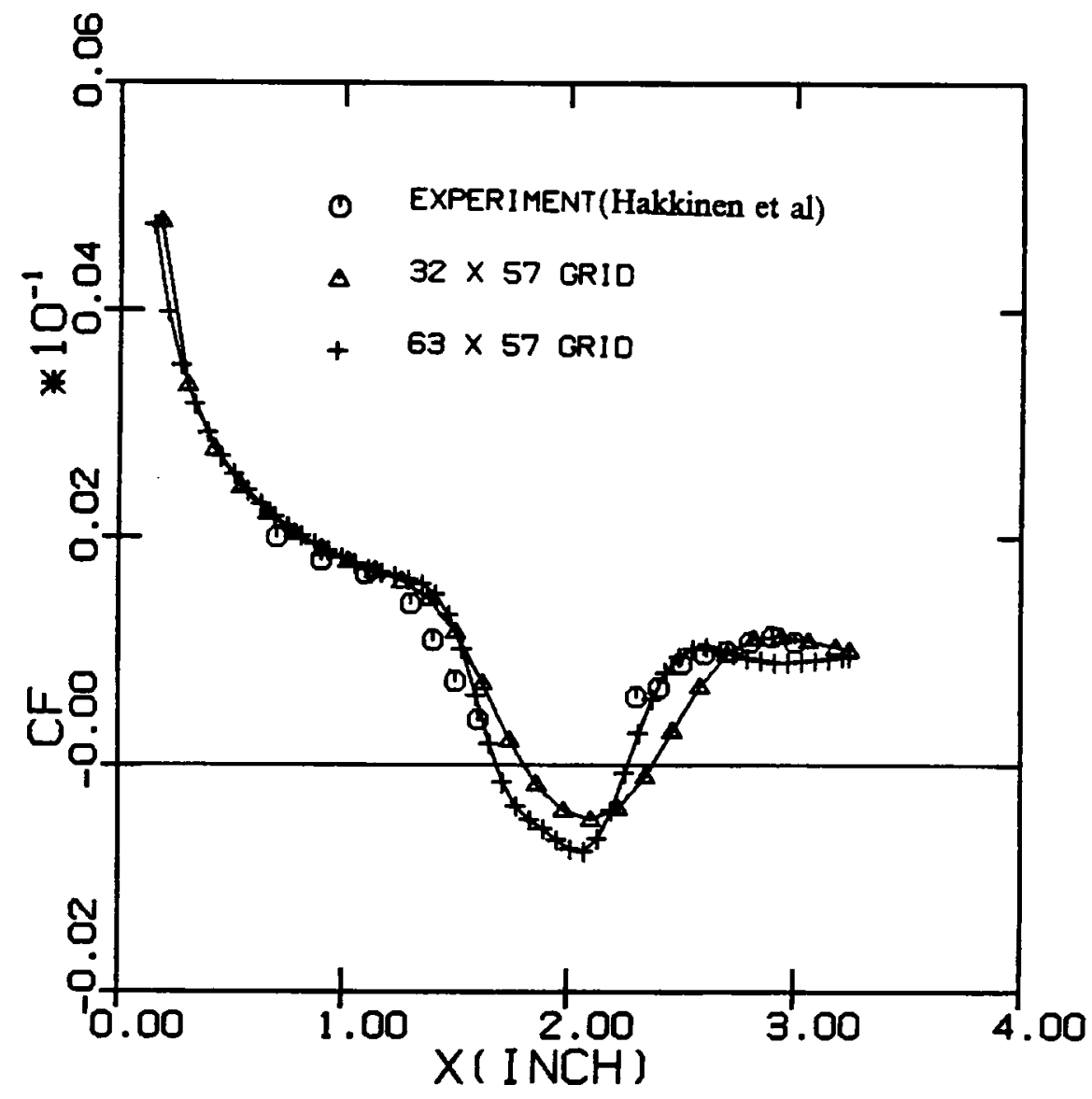

Figure 26. Grid refinement for the laminar boundary layer - shock interaction, skin friction (FVS-van Leer). 


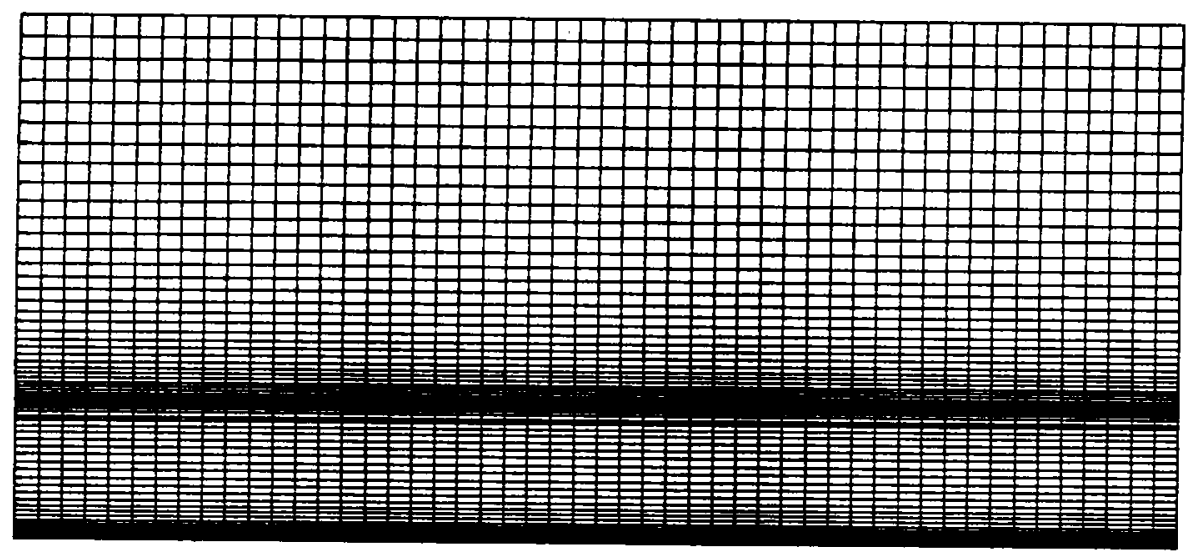

Figure 27. $51 \times 70$ grid for supersonic slot injection problem (without wedge). 


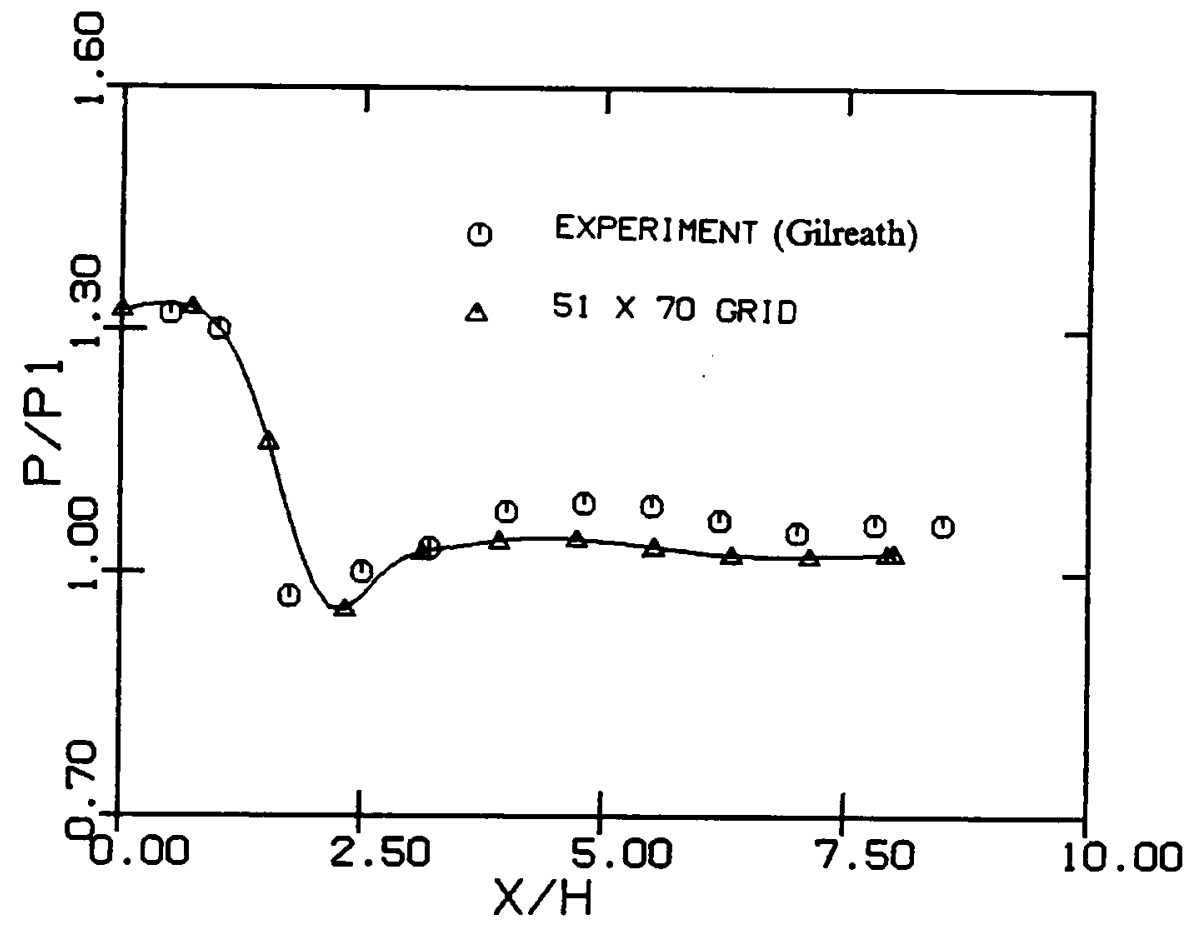

Figure 28. Wall pressure distribution for supersonic slot injection problem (without wedge). 


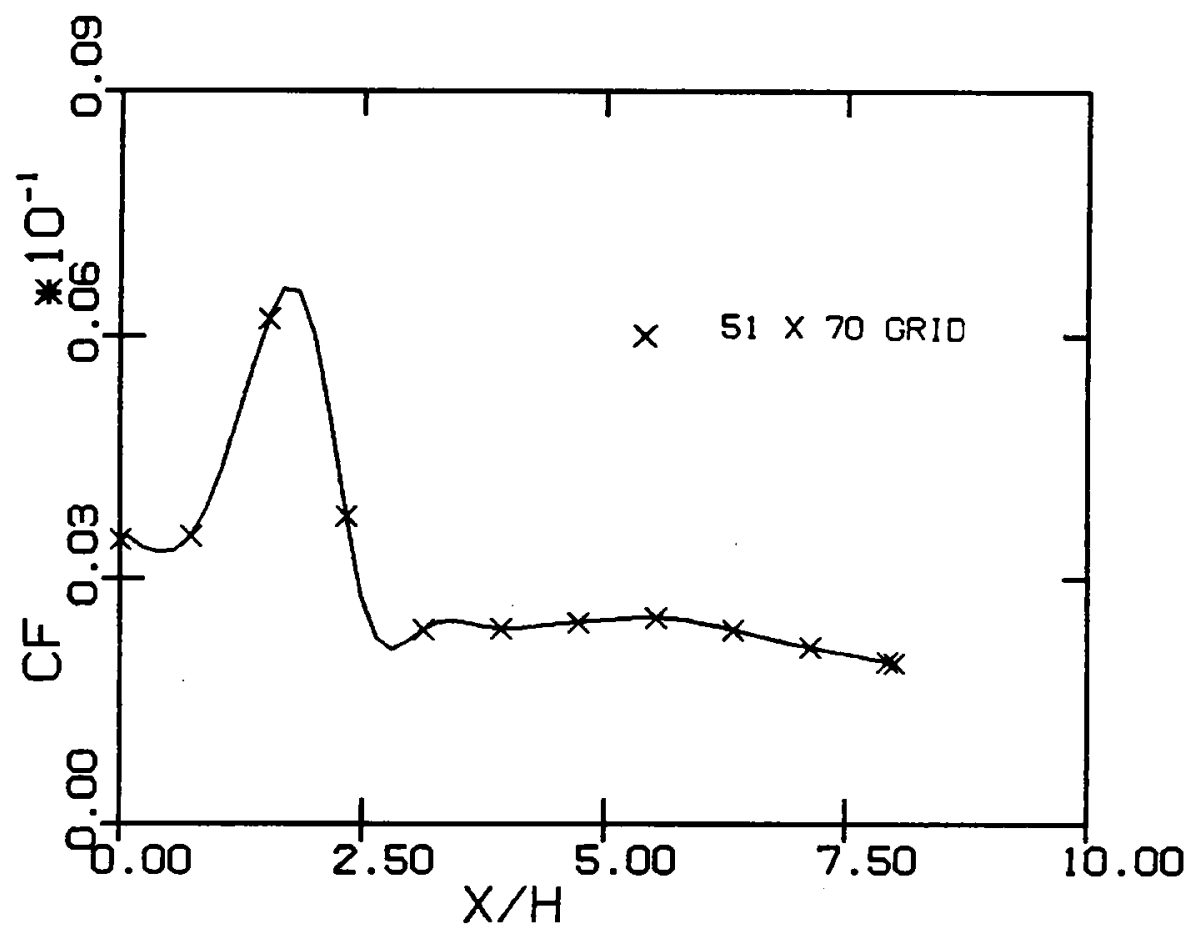

Figure 29. Skin friction distribution for supersonic slot injection problem (without wedge). 


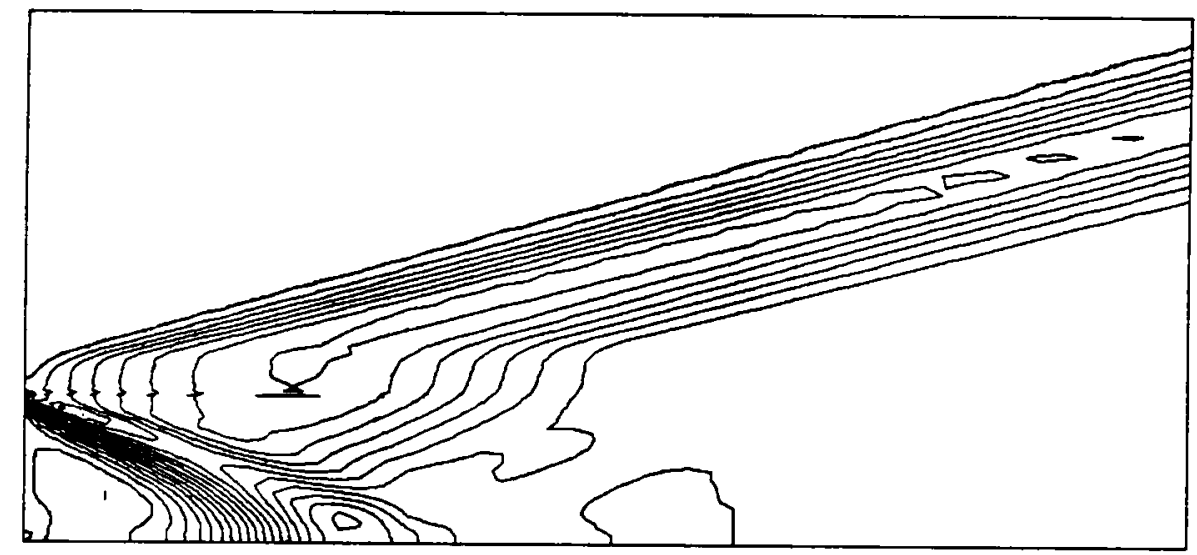

Figure 30. Pressure contour for supersonic slot injection problem (without wedge) - $51 \times 70$ grid. 


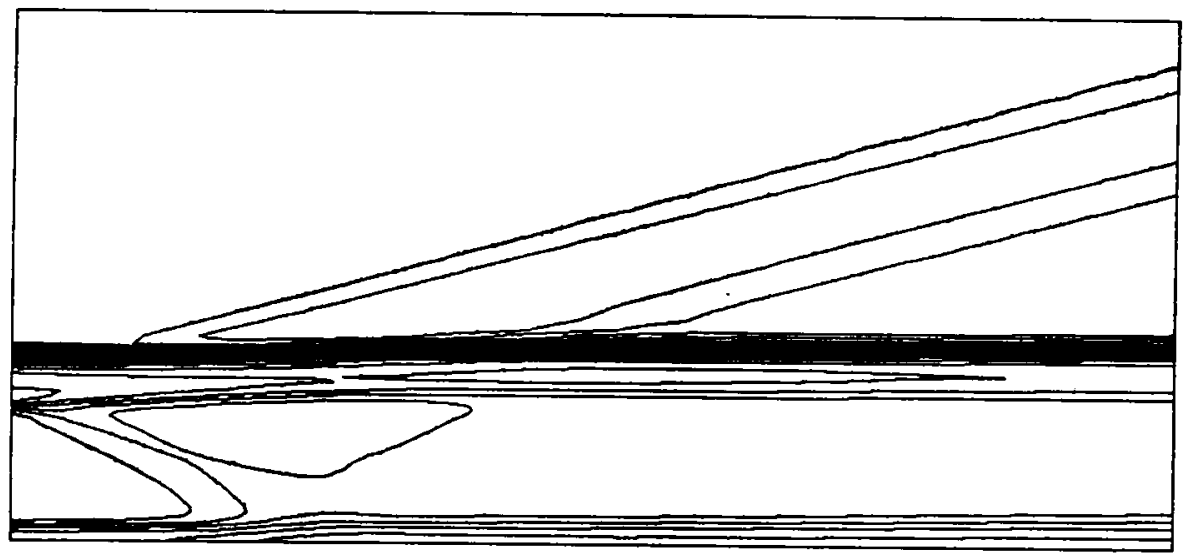

Figure 31. Density contour for supersonic slot injection problem (without wedge) - $51 \times 70$ grid. 


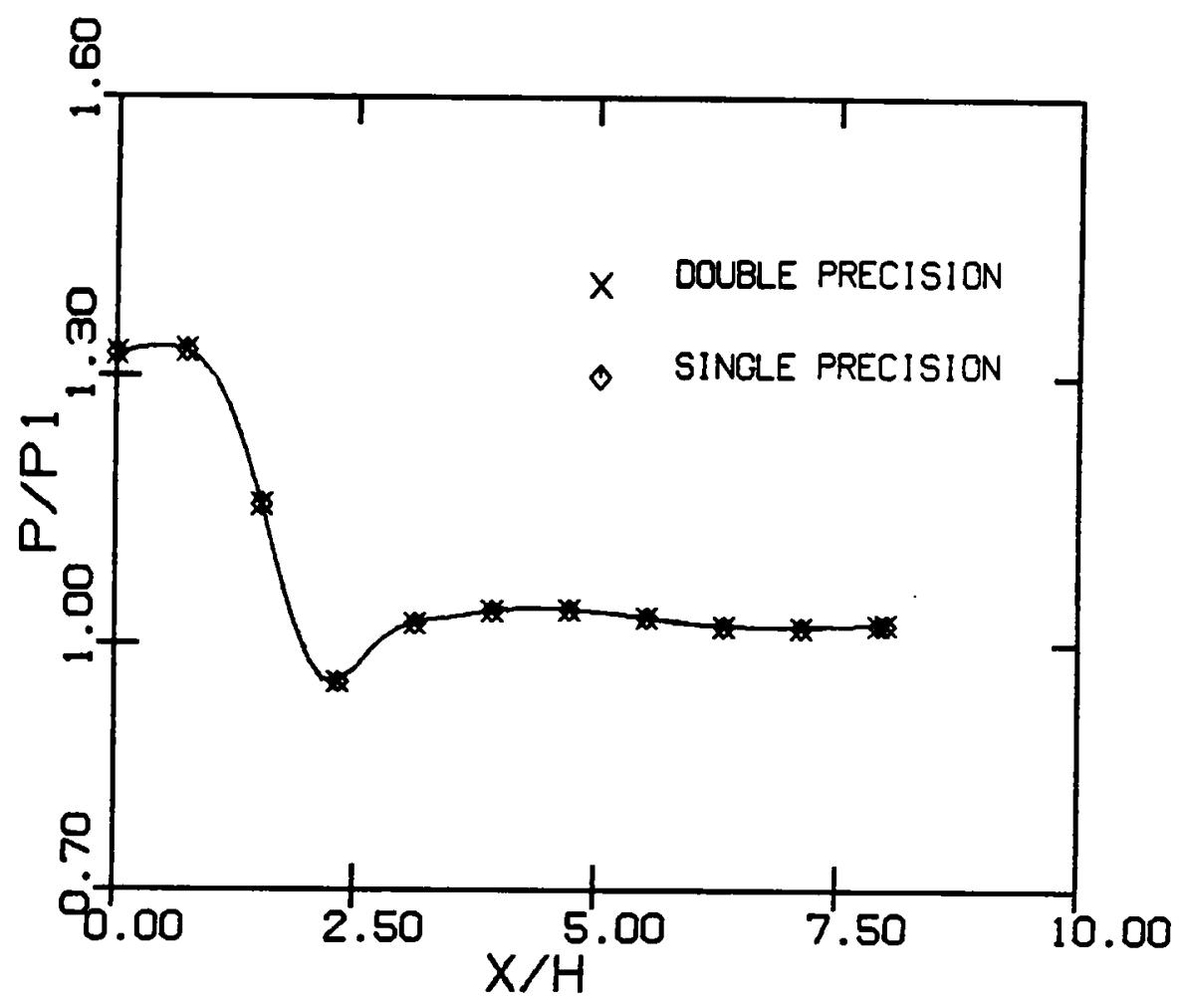

Figure 32. Wall pressure distribution for supersonic slot injection problem ( $51 \times 70$ grid ) - double precision vs. single precision. 


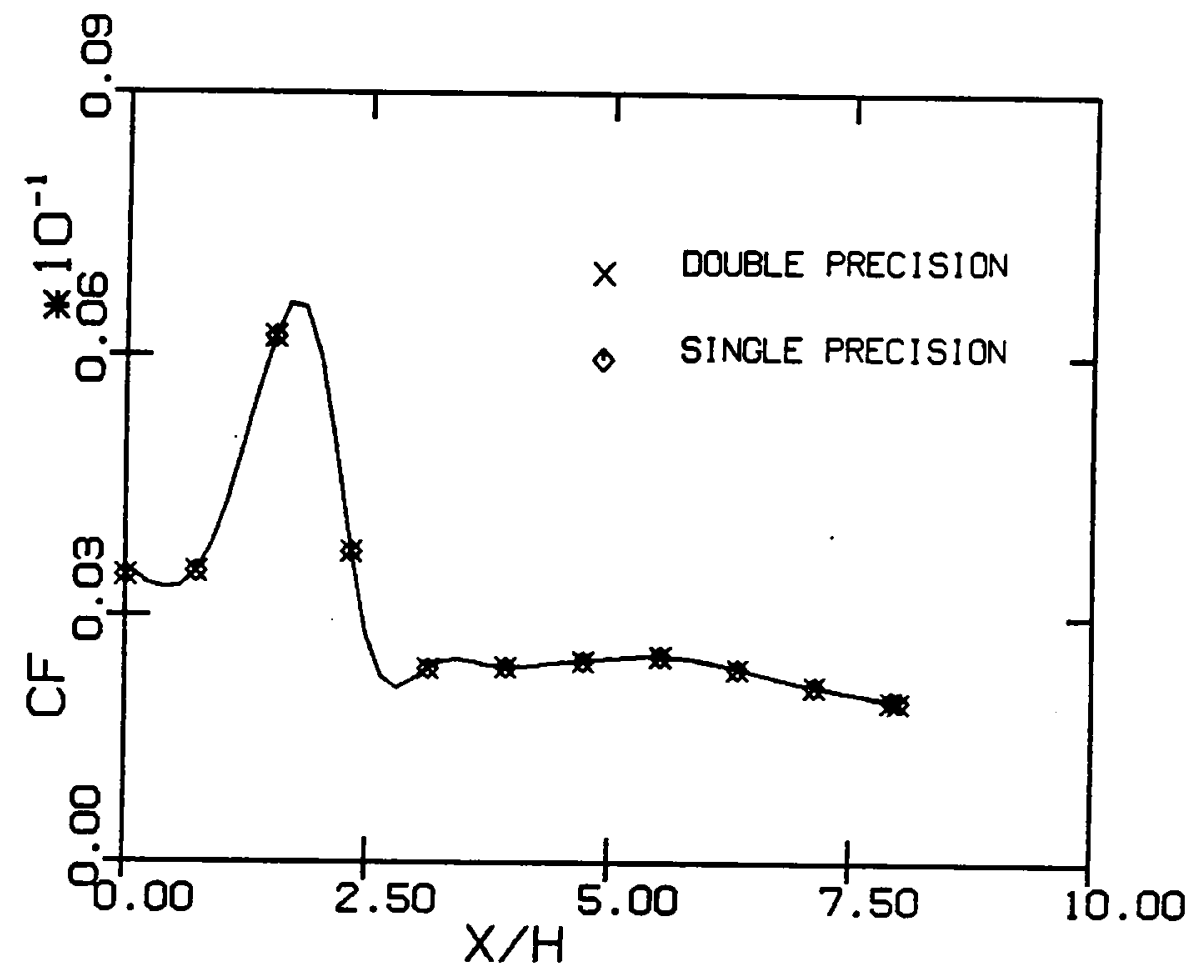

Figure 33. Skin friction distribution for supersonic slot injection problem ( $51 \times 70$ grid ) - double precision vs. single precision. 


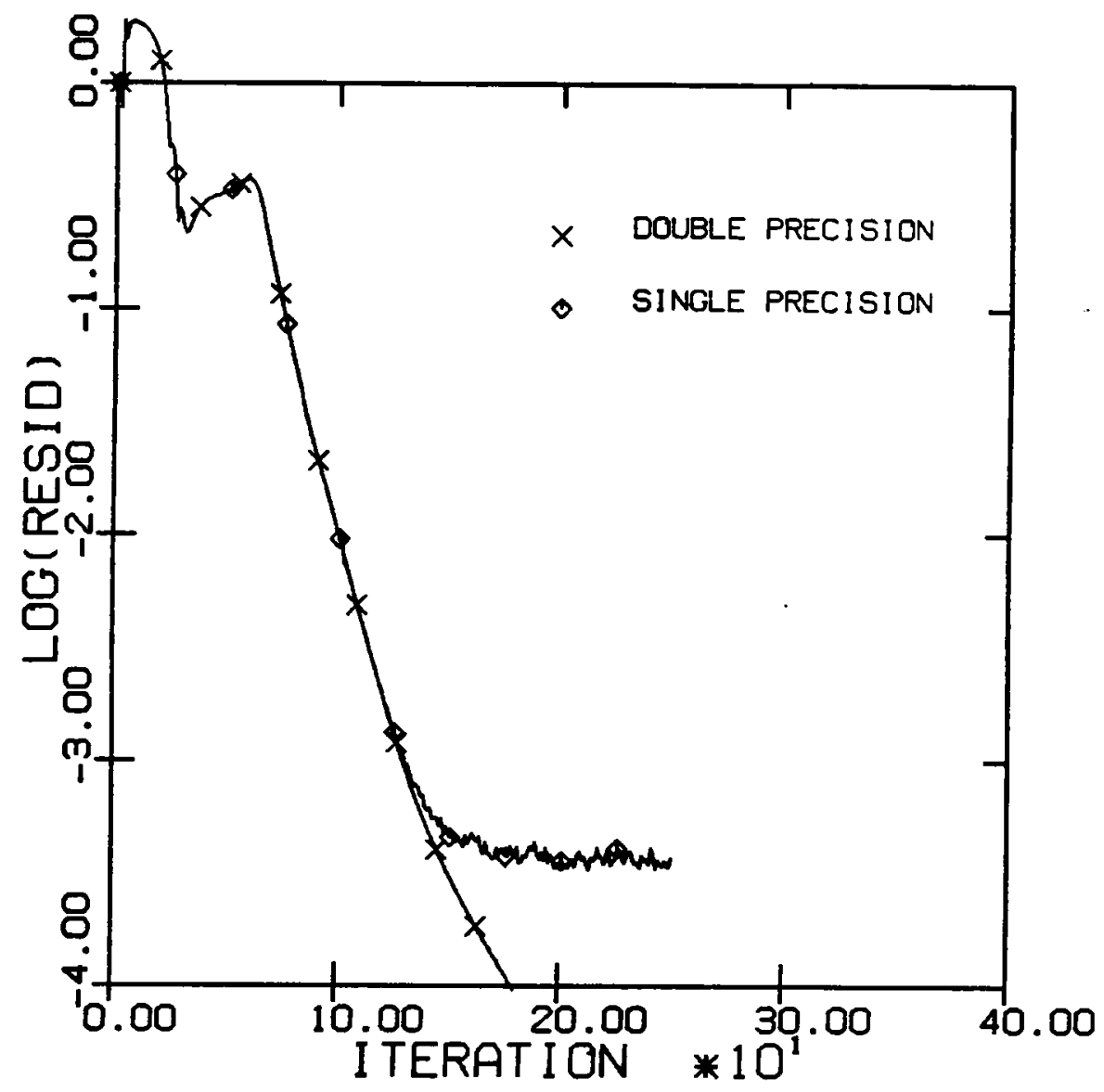

Figure 34. Convergence history for supersonic slot injection problem ( $51 \mathrm{x}$ 70 grid ) - double precision vs. single precision. 


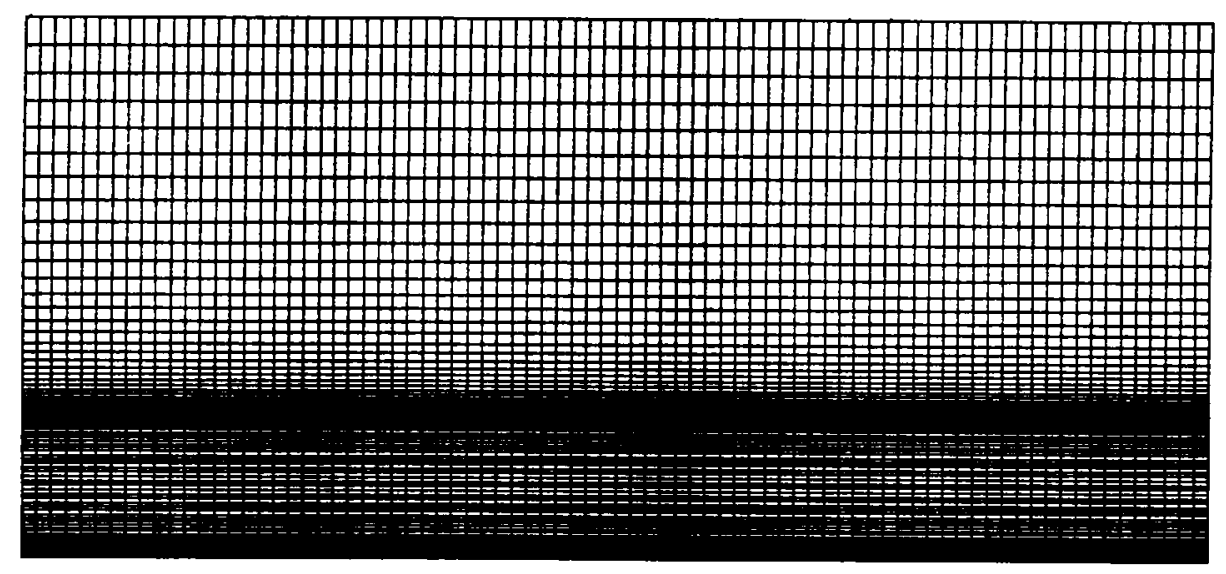

Figure 35. $81 \times 90$ grid for supersonic slot injection problem (without wedge). 


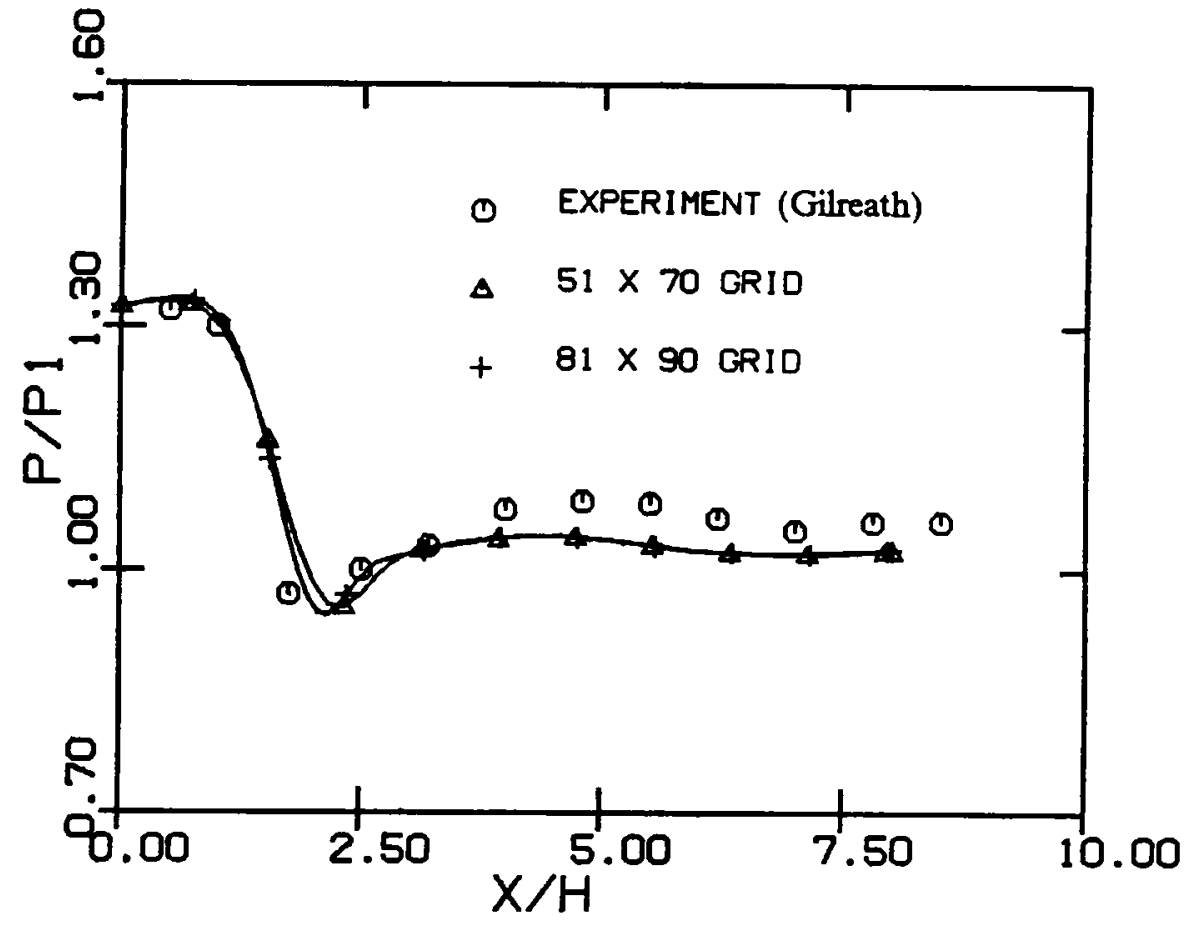

Figure 36. Grid refinement for supersonic slot injection problem (without wedge) - wall pressure. 


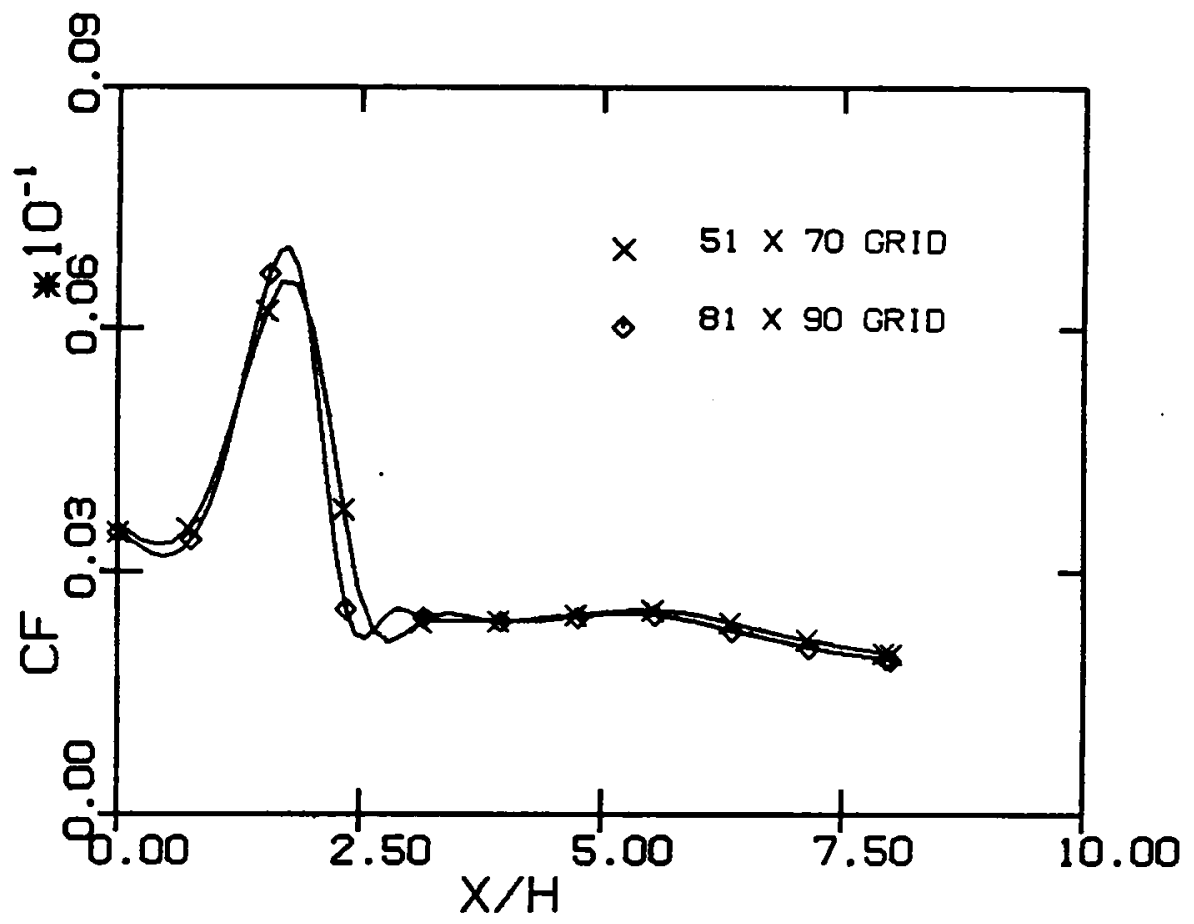

Figure 37. Grid refinement for supersonic slot injection problem (without wedge) - skin friction. 


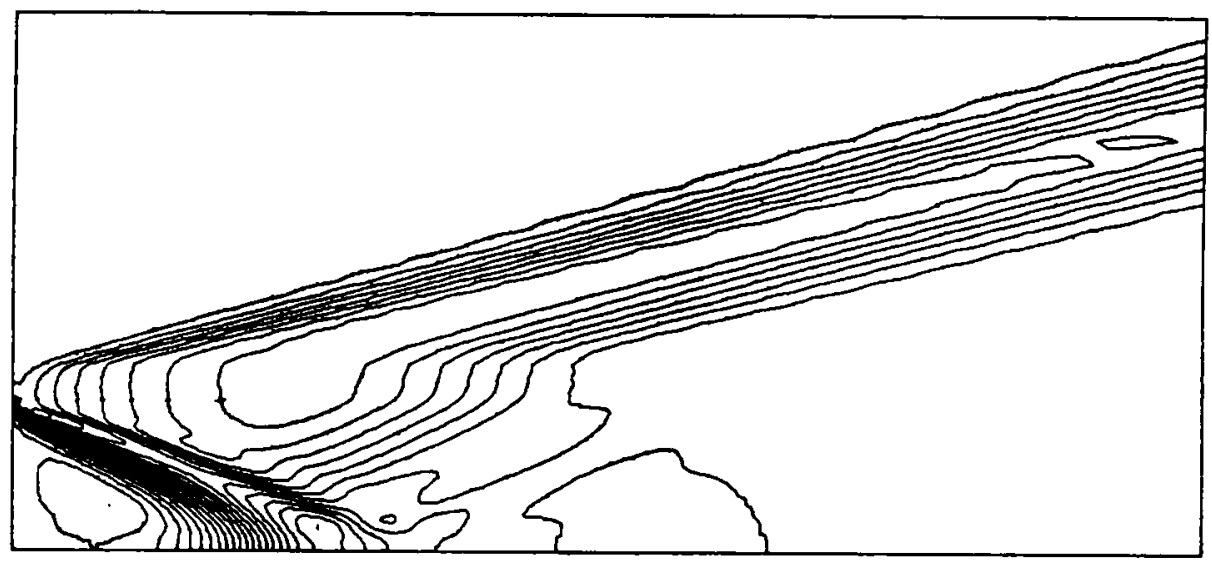

Figure 38. Pressure contour for supersonic slot injection problem (without wedge) $-81 \times 90$ grid. 


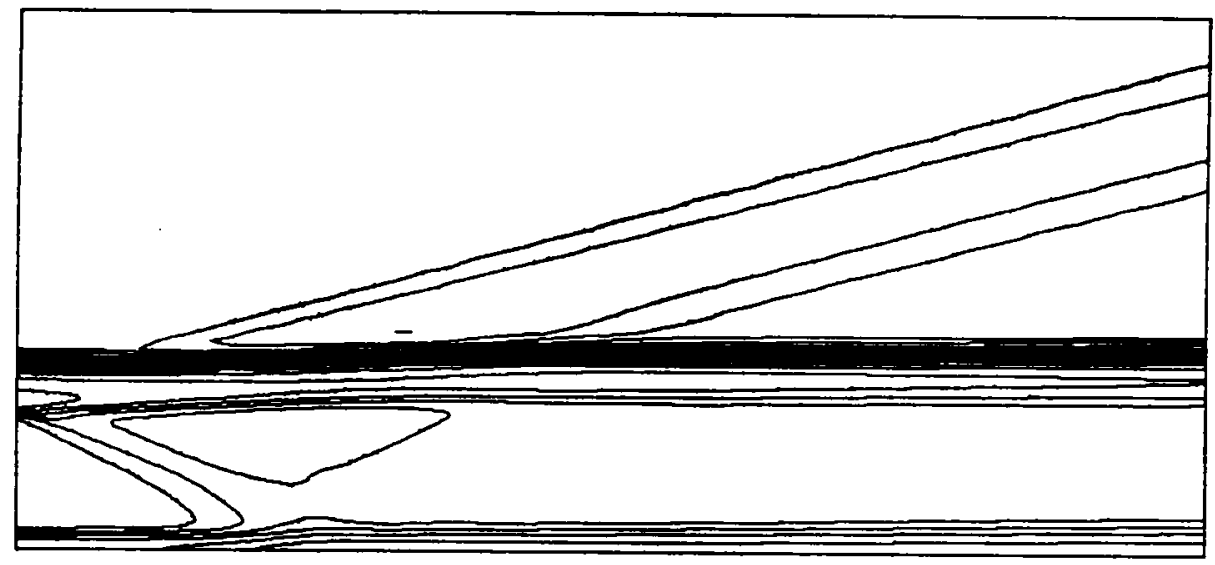

Figure 39. Density contour for supersonic slot injection problem (without wedge) $-81 \times 90$ grid. 


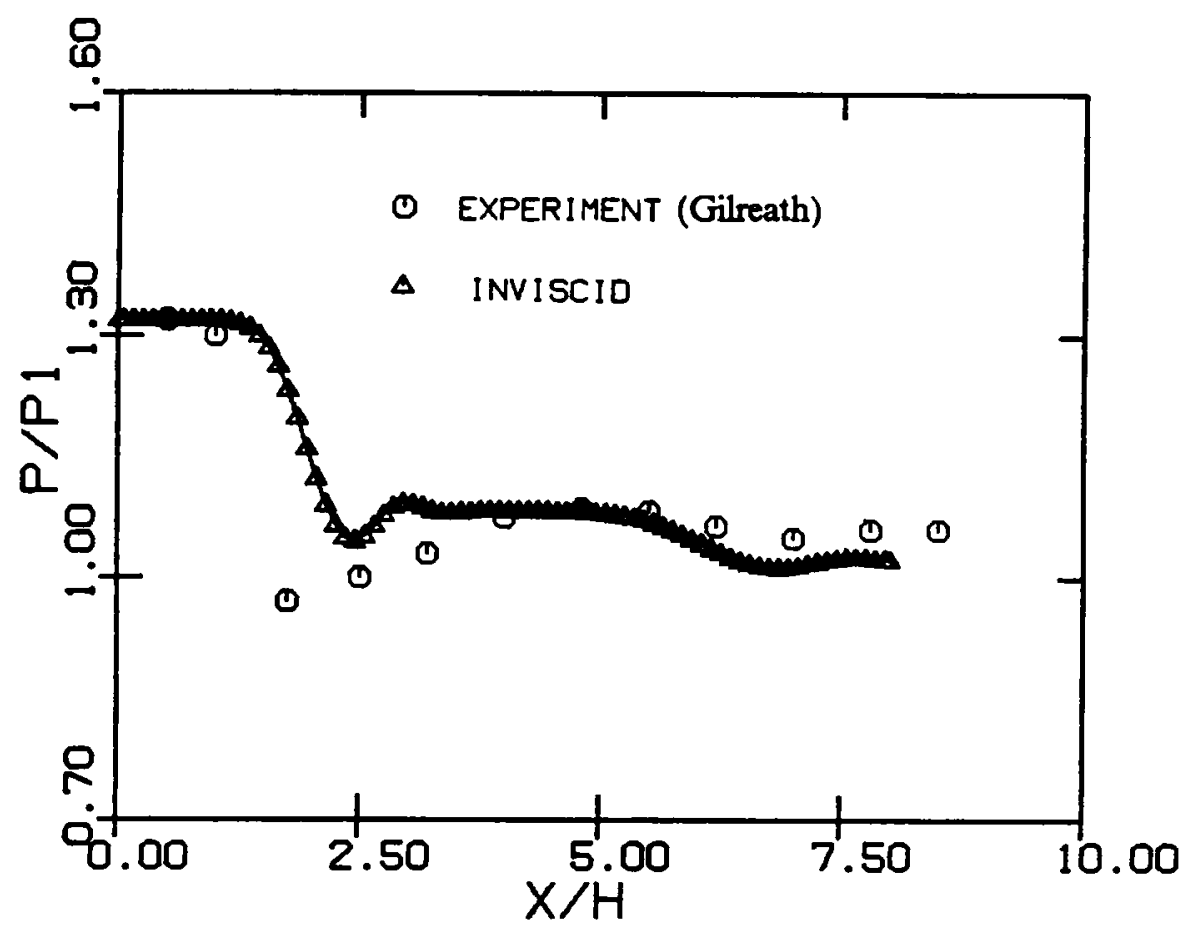

Figure 40. Wall pressure distribution for supersonic slot injection problem (without wedge), inviscid - $81 \times 90$ grid. 


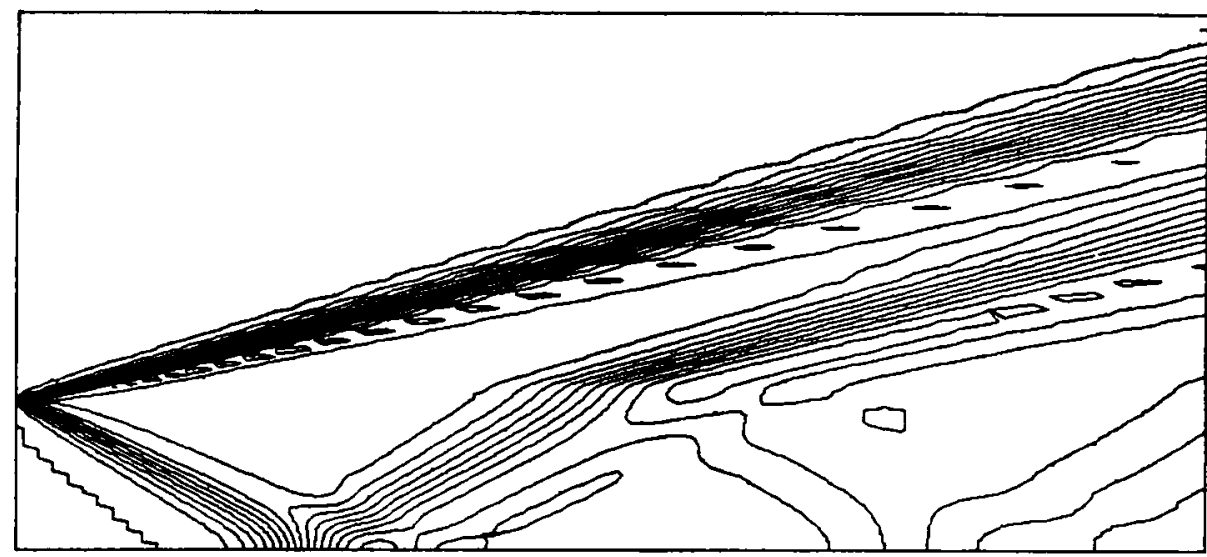

Figure 41. Pressure contour for supersonic slot injection problem (without wedge), inviscid - $81 \times 90$ grid. 


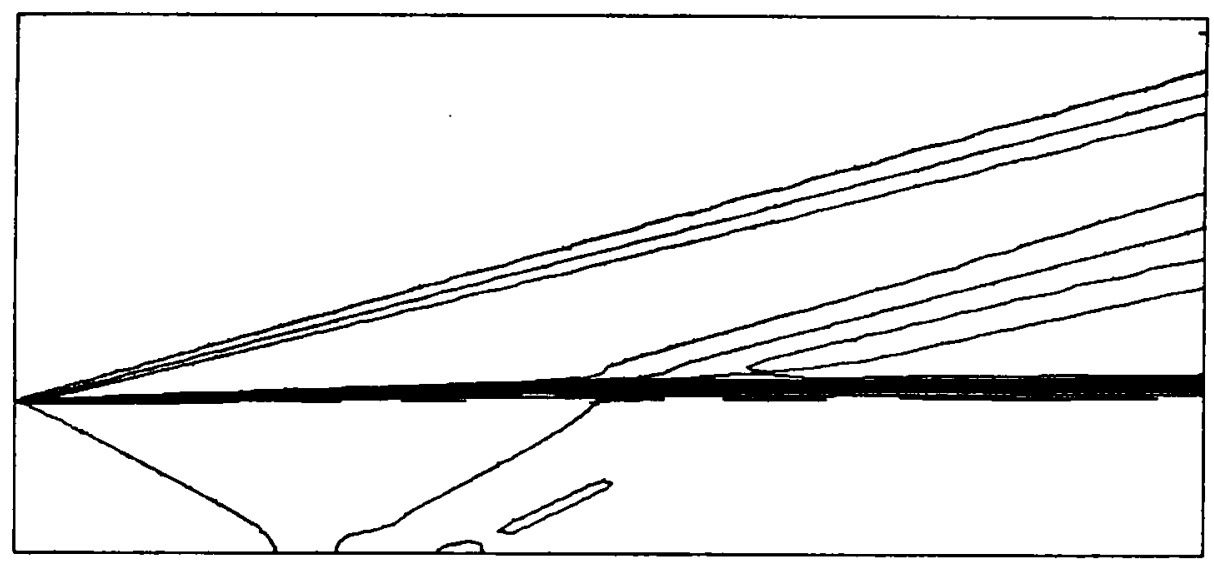

Figure 42. Density contour for supersonic slot injection problem (without wedge), inviscid - $81 \times 90$ grid. 


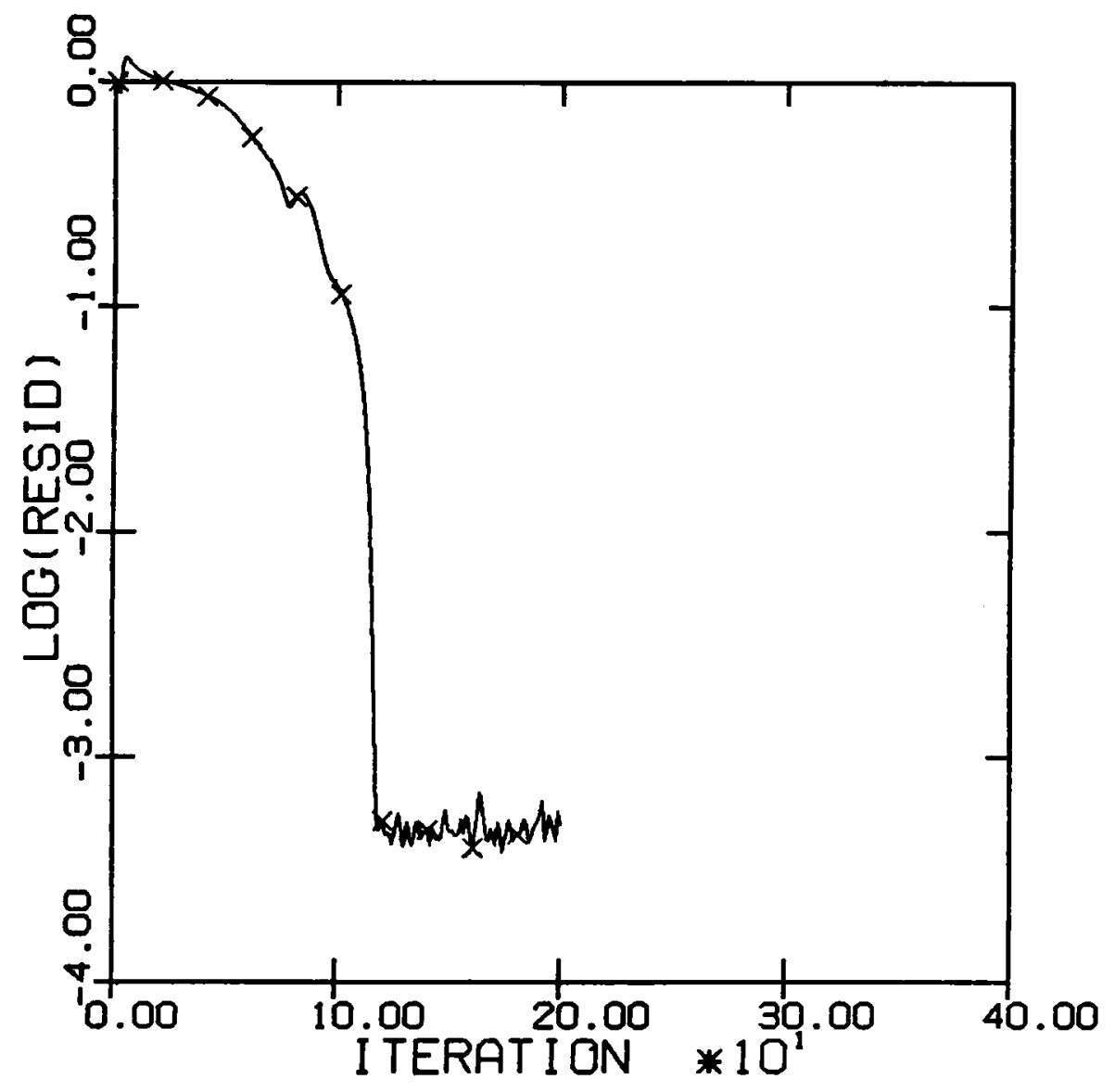

Figure 43. Convergence history for supersonic slot injection problem (without wedge), inviscid - $81 \times 90$ grid. 


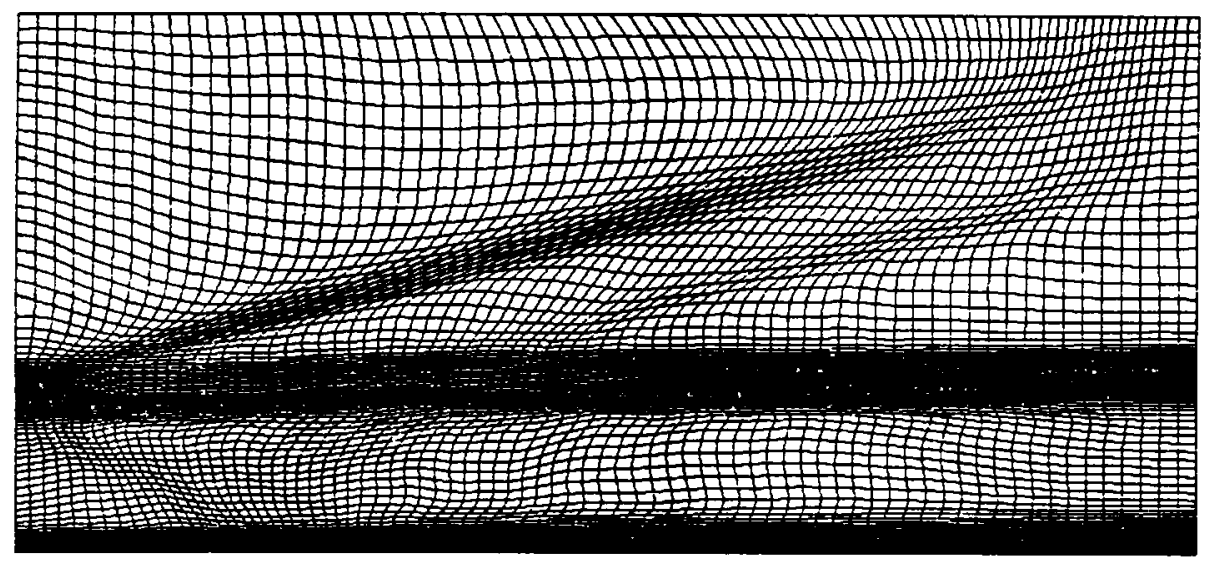

Figure 44. $81 \times 90$ adaptive grid for supersonic slot injection problem (without wedge). 


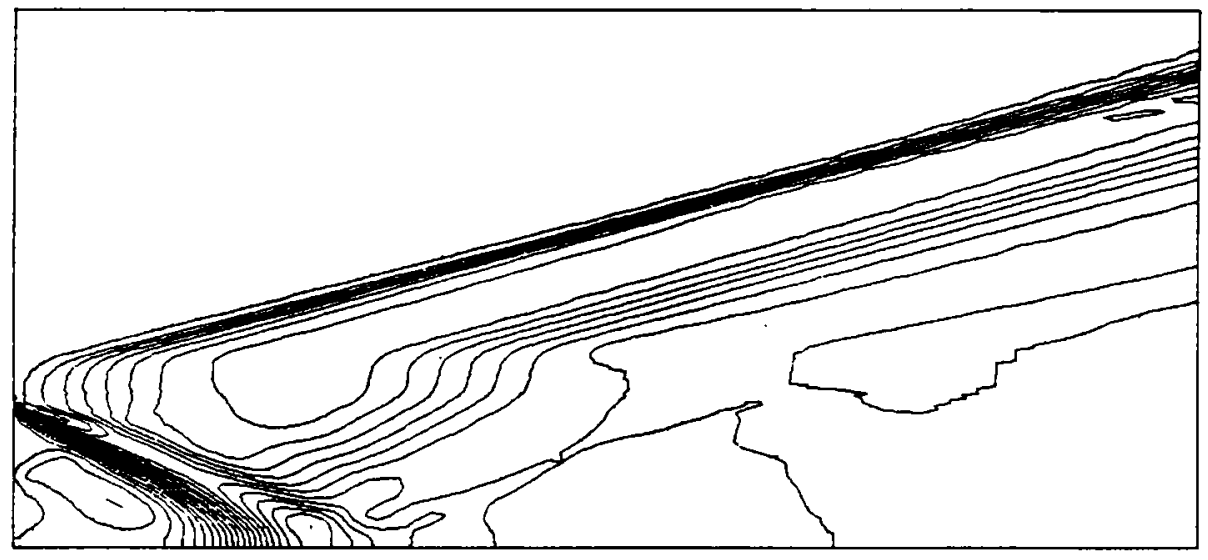

Figure 45. Pressure contour for supersonic slot injection problem (without wedge) $-81 \times 90$ adaptive grid. 


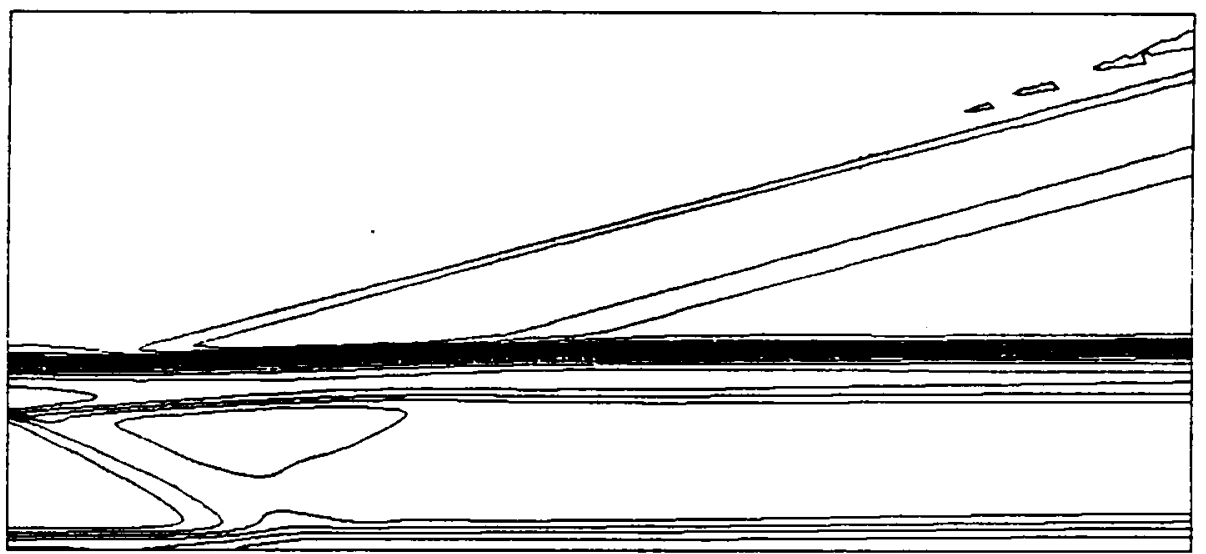

Figure 46. Density contour for supersonic slot injection problem (without wedge) - $81 \times 90$ adaptive grid. 


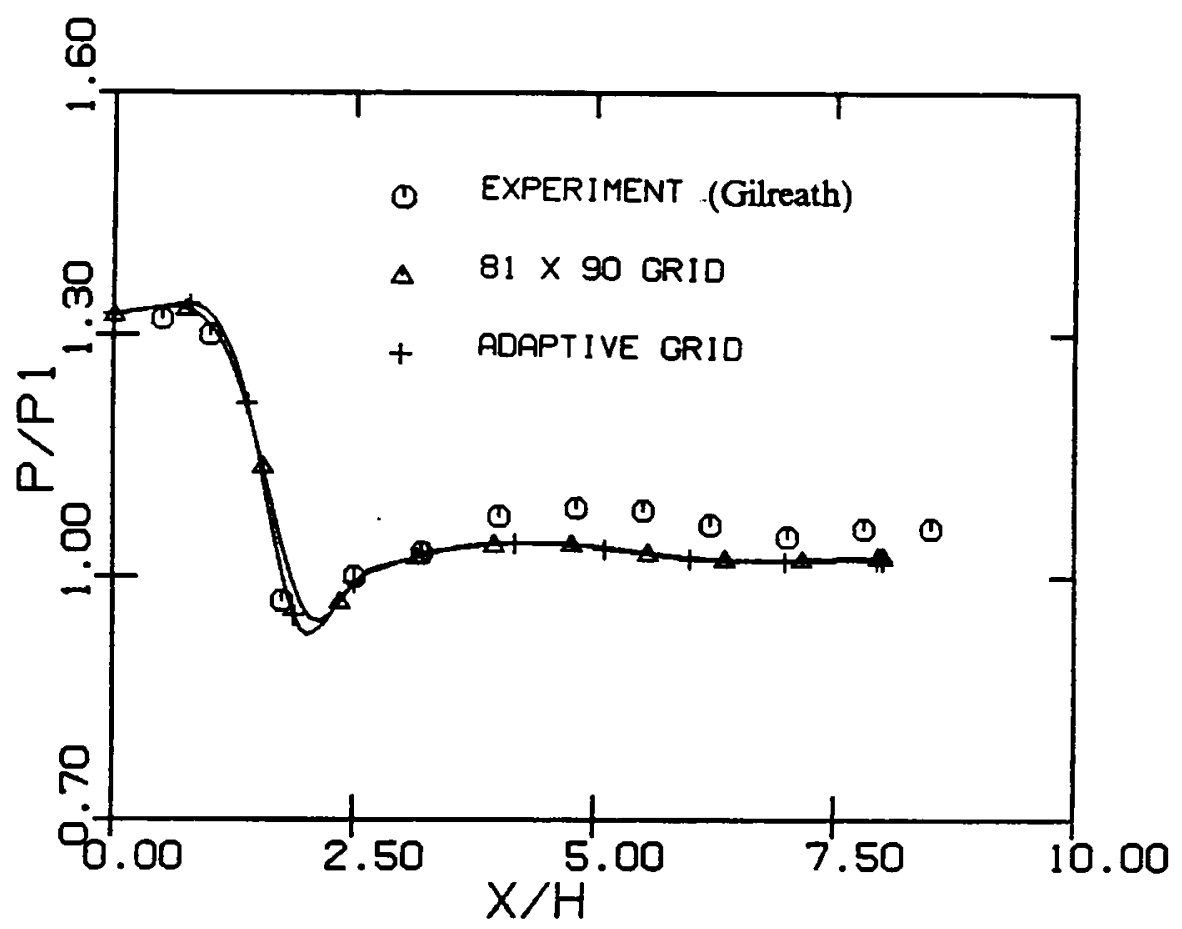

Figure 47. Wall pressure distribution for supersonic slot injection problem (without wedge) $-81 \times 90$ adaptive grid. 


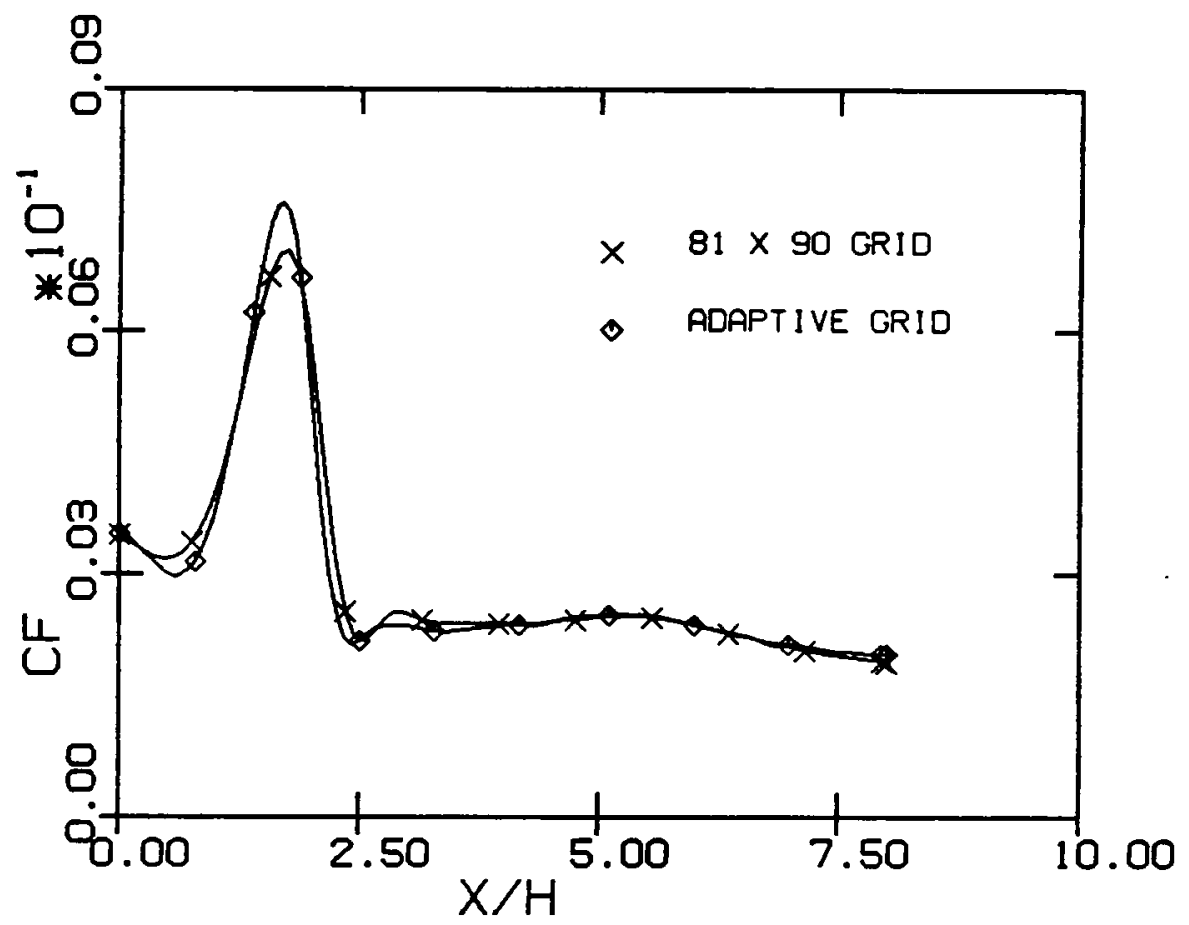

Figure 48. Wall skin friction distribution for supersonic slot injection problem (without wedge) - $81 \times 90$ adaptive grid. 


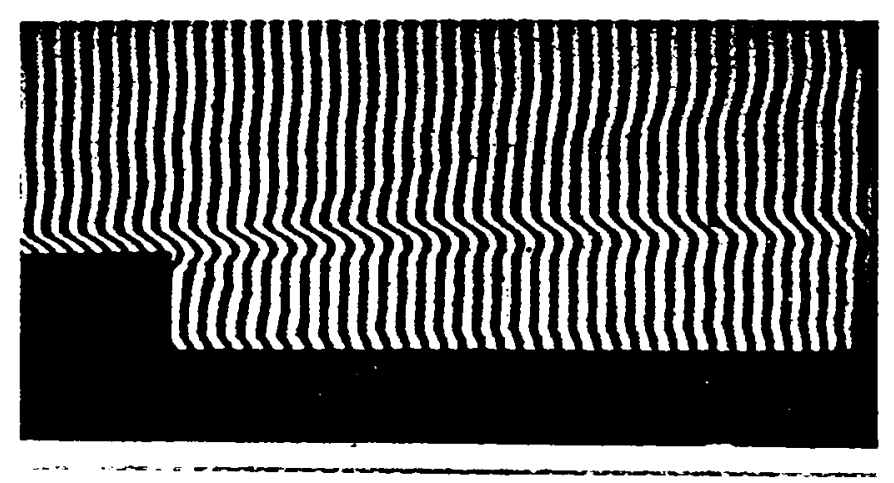

a) experimental interferogram (Gilreath)

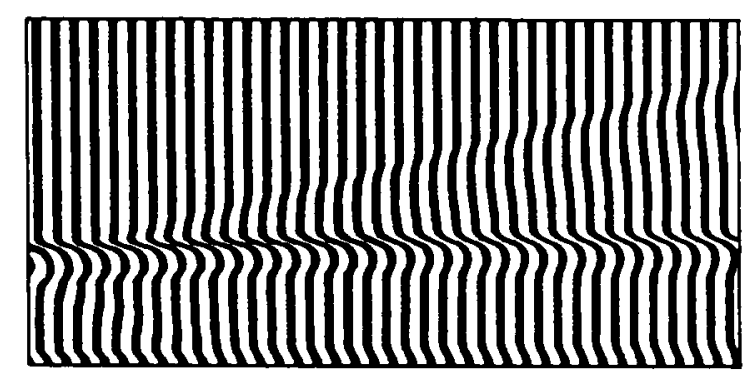

b) numerical interferogram

Figure 49. Comparison of experimental and numerical flowfield visualization (without wedge) - interferograms. 


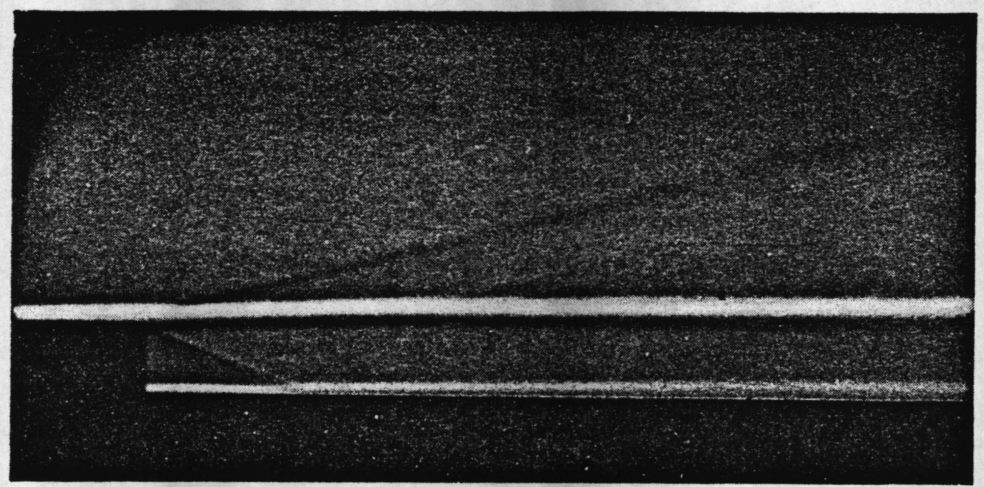

a) experimental Schlieren (Gilreath)

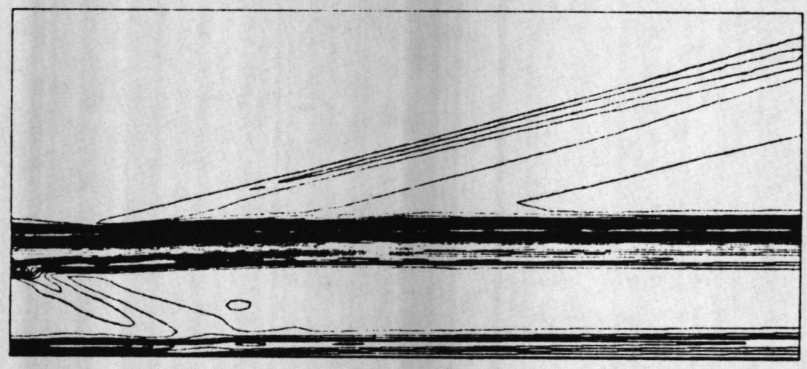

b) numerical Schlieren (isolines of $\frac{\partial \rho}{\partial y}$ )

Figure 50. Comparison of experimental and numerical flowfield visualization (without wedge) - Schlieren. 


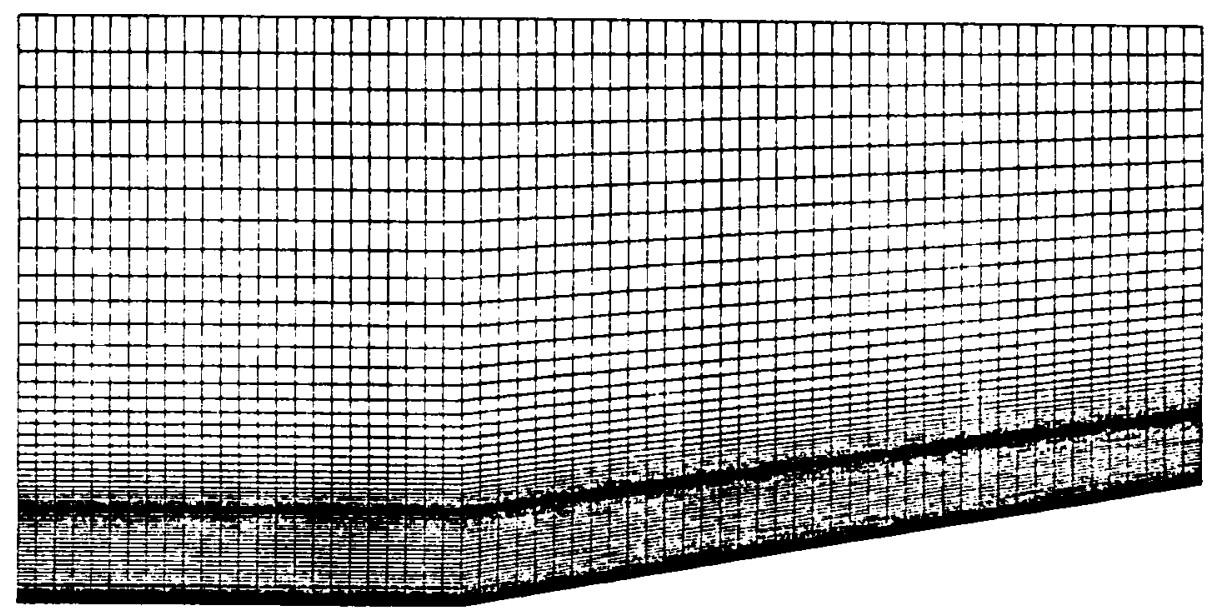

Figure 51. $65 \times 70$ grid for supersonic slot injection over a 10 degree wedge surface. 


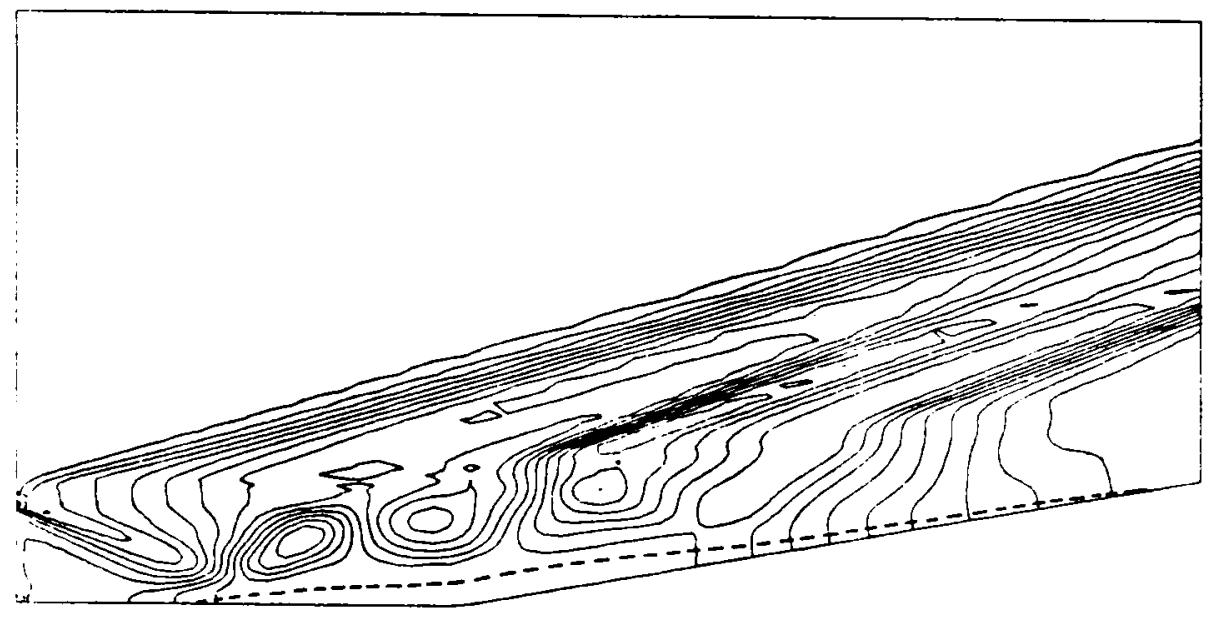

Figure 52. Pressure contour for supersonic slot injection over a 10 degree wedge surface. 


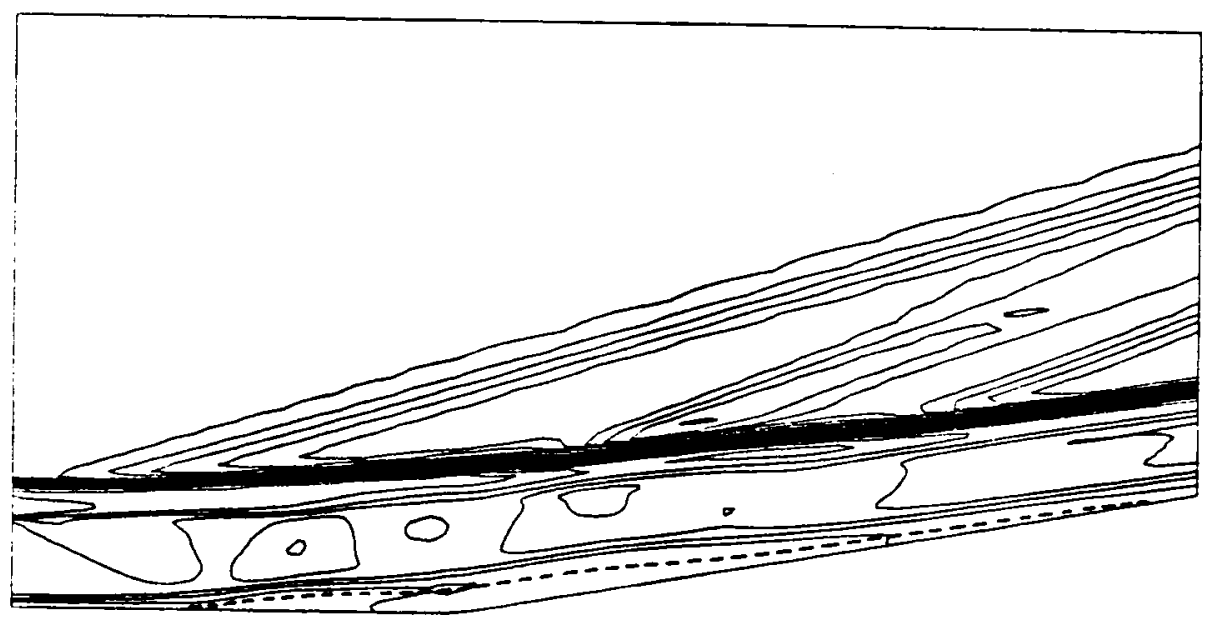

Figure 53. Density contour for supersonic slot injection over a 10 degree wedge surface. 


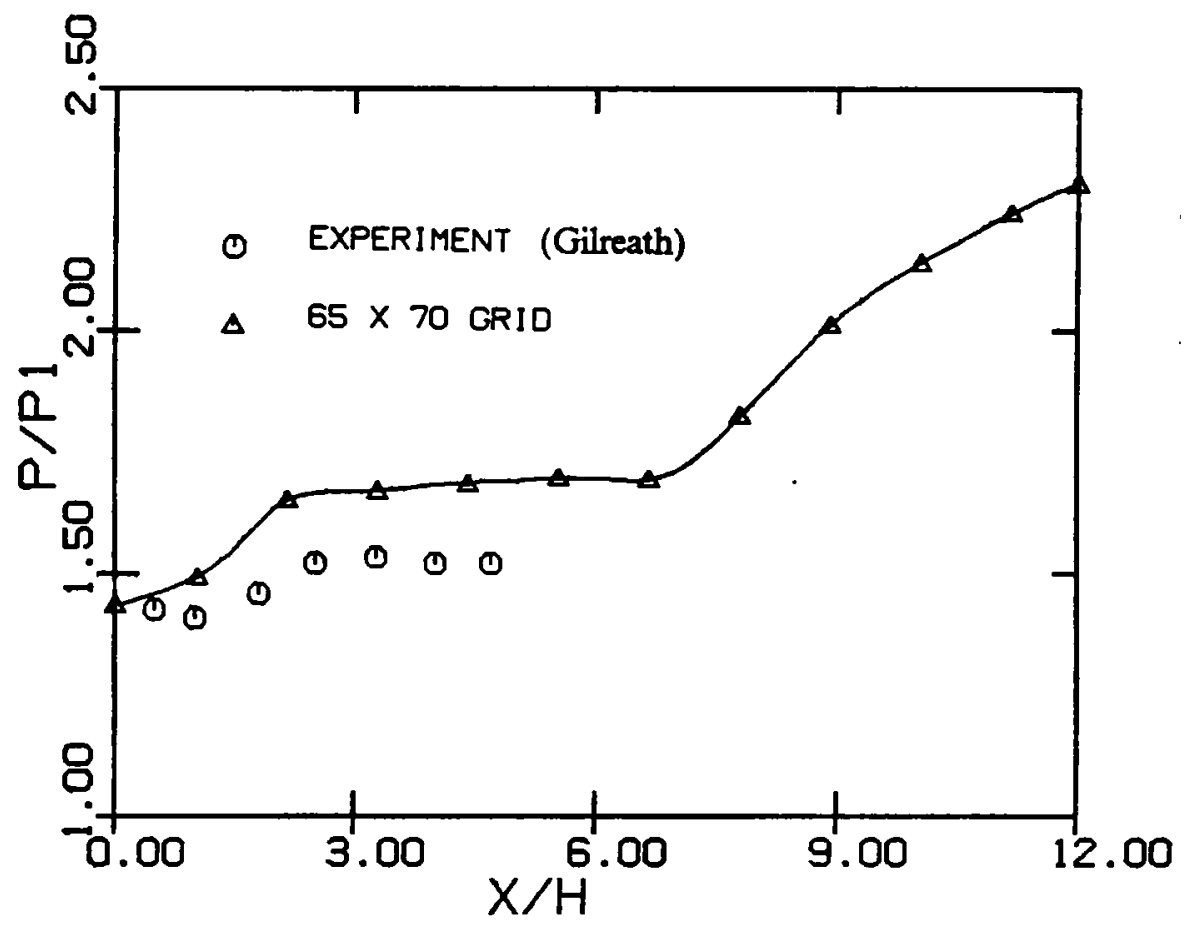

Figure 54. Comparison of wall pressure distribution between numerical and experimental data (with 10 degree wedge). 


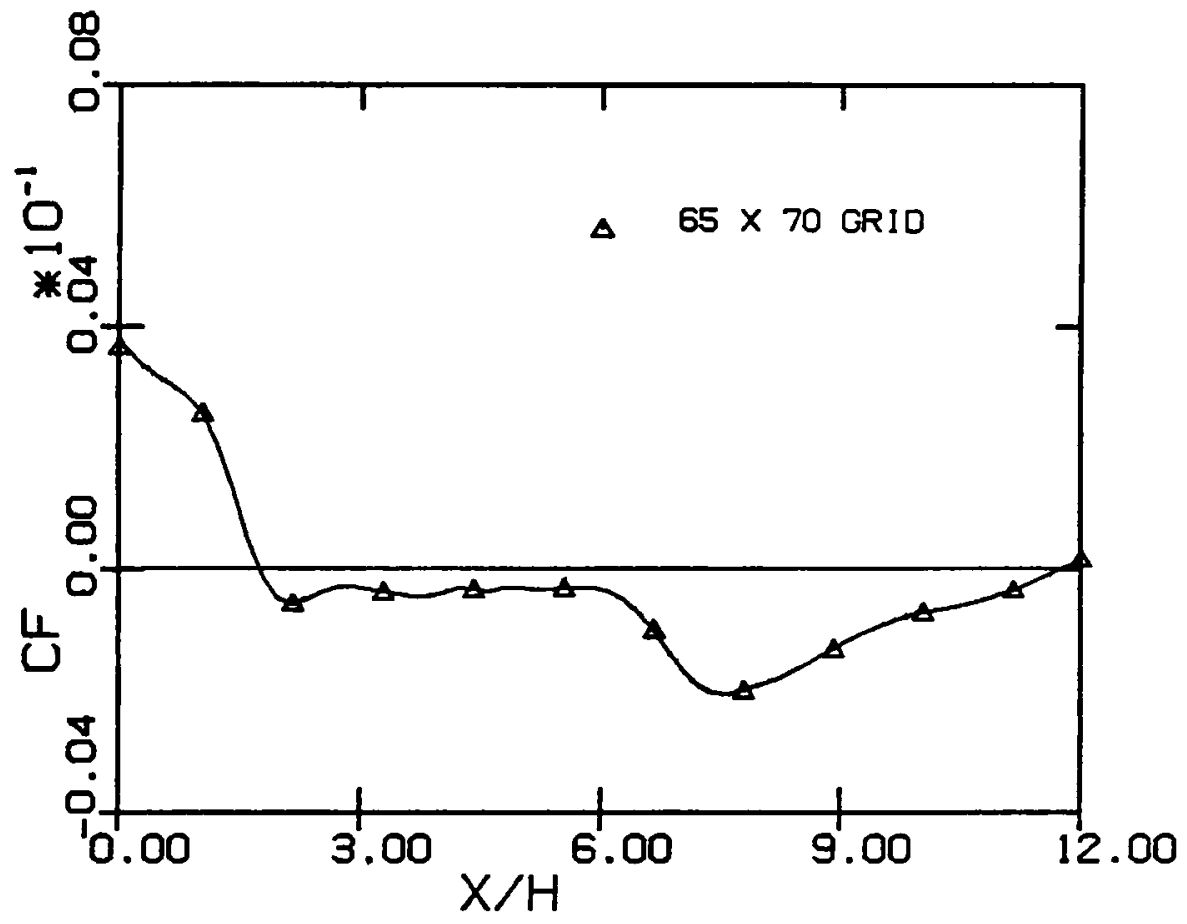

Figure 55. Skin friction distribution (with 10 degree wedge) $-65 \times 70$ grid. 


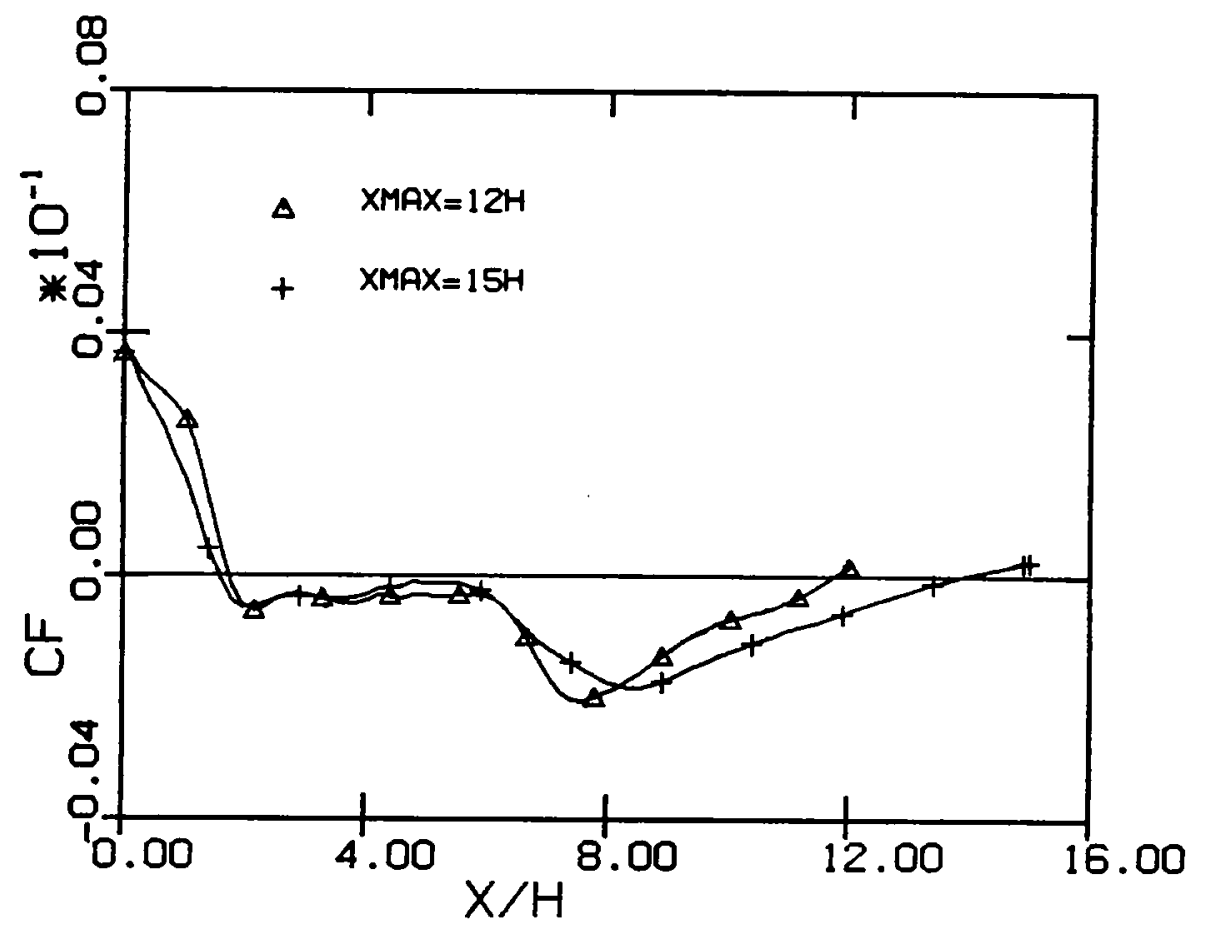

Figure 56. The effect of the location of outflow boundary - skin friction. 


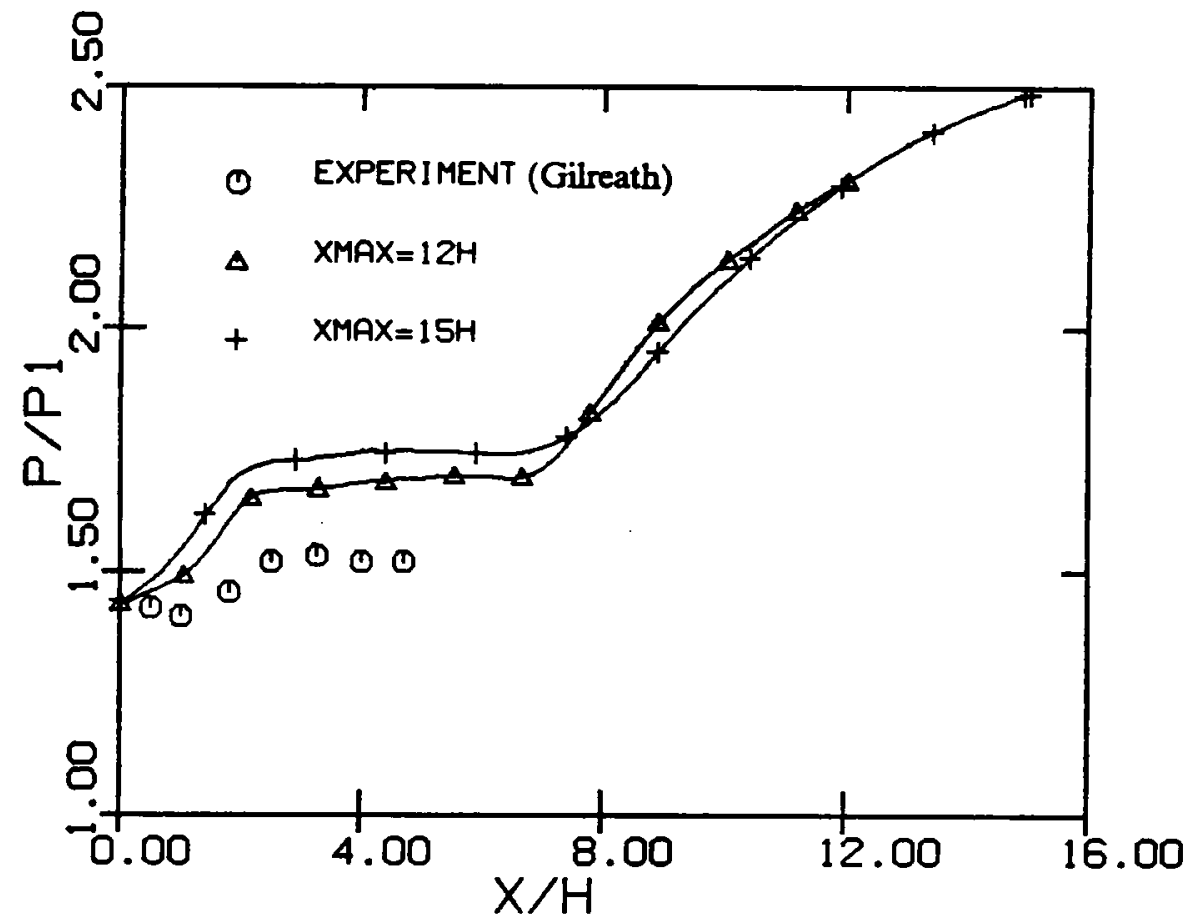

Figure 57. The effect of the location of outflow boundary - pressure. 


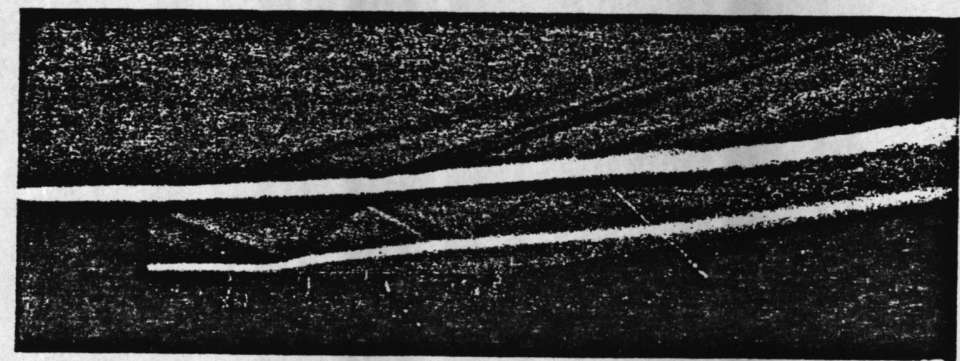

a) experimental Schlieren (Gilreath)

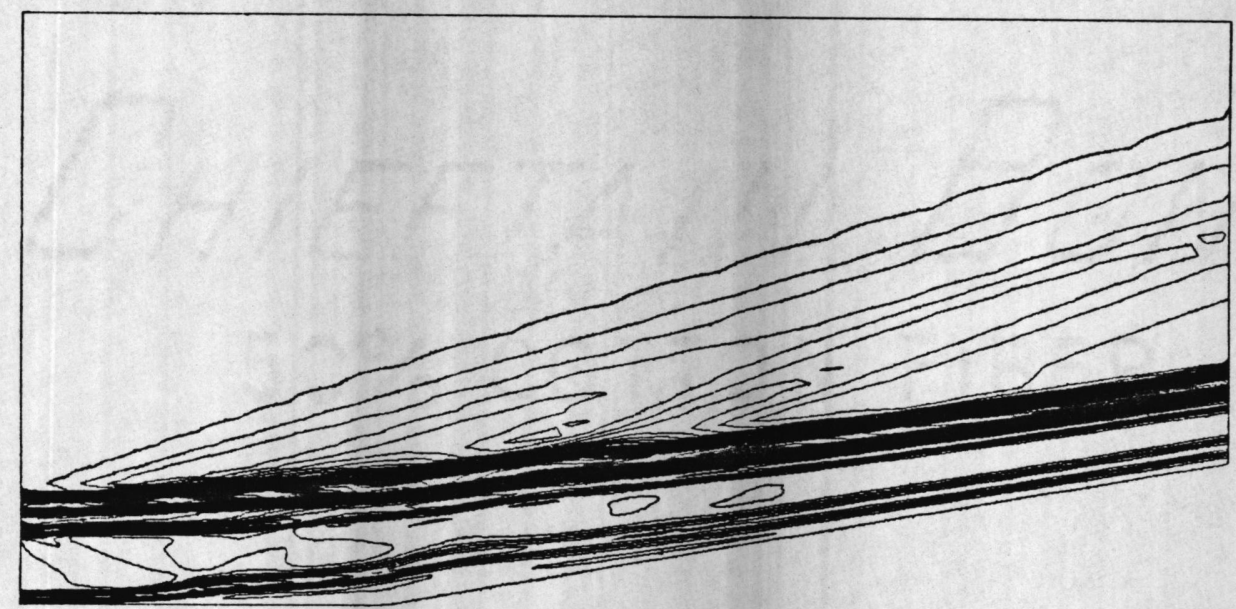

b) numerical Schlieren (isolines of $\frac{\partial \rho}{\partial y}$ )

Figure 58. Comparison of experimental and numerical flowfield visualization (with 10 degree wedge) - Schlieren. 


\section{The vita has been removed from the scanned document}

